\title{
The Physics of Positron Annihilation in the Solar Atmosphere
}

\author{
R. J. Murphy and G. H. Share \\ E. O. Hulburt Center for Space Research, Code 7652, Naval Research Laboratory, \\ Washington, DC 20375-5352 \\ J. G. Skibo \\ 2820 Eliston St., Bowie, MD 20716 \\ B. Kozlovsky \\ School of Physics and Astronomy, Tel Aviv University, Ramat Aviv, Israel
}

\begin{abstract}
We compile the most recently measured and calculated cross sections for processes relevant to annihilation of positrons in solar flares and present the final evaluated cross sections. We calculate the $511 \mathrm{keV}$ annihilation line spectrum and relative strength of the $3 \gamma$ continuum for each of the annihilation processes, using a Monte Carlo code that simulates the thermalization of and positronium production by $\sim \mathrm{MeV}$ positrons. We calculate the thermally-averaged annihilation and positronium production rates and the positronium quenching rates. We apply the results to four specific environments (fully ionized, neutral, partially ionized and a non-LTE model of the quiet solar atmosphere), calculating the relative strengths of each process and the combined total annihilation line spectrum. The results are compared with data obtained recently from the high spectralresolution detectors of RHESSI. We find that positron annihilation in solar flares can occur in a wide variety of environments. The cross sections presented here are also useful for evaluation of positron annihilation in other environments such as the interstellar medium.
\end{abstract}

Subject headings: Sun: Flares — Sun: X-rays, Gamma Rays - Atomic Processes - Line: Formation

\section{Introduction}

Positron annihilation radiation from solar flares was first observed by Chupp et al. (1973). Subsequently, more detailed observations of the line from many solar flares were 
made with the medium energy-resolution Solar Maximum Mission (SMM) NaI spectrometer (Share et al. (1983, 1996); see also the flare catalog of Vestrand et al. (1999)). The early observational data included the total yield and time history of the radiation (Share et al. 1983; Murphy et al. 1984)). Recently, the Reuven Ramaty High Energy Solar Spectroscopic Imager (RHESSI) observed the solar annihilation line for the first time with high energyresolution Ge detectors (Share et al. 2003) allowing detailed investigation of its spectral shape.

The line has now been observed by RHESSI in several flares (Share et al. 2004) and has been found to be broad (4-8 keV), consistent with annihilations in an ambient ionized medium at temperatures above $10^{5} \mathrm{~K}$. Except possibly for the first 4 minutes of one flare, the measured continuum from positronium was unobservable, indicating that the density at which most annihilations occurred was greater than $\sim 10^{14} \mathrm{H} \mathrm{cm}^{-3}$. The width of the line narrowed in 2 minutes to $<1 \mathrm{keV}$ late in this flare, consistent with annihilation in ionized $\mathrm{H}$ at $T<10^{4} \mathrm{~K}$ and density $>10^{15} \mathrm{~cm}^{-3}$. These observations suggest a highly dynamic flaring atmosphere at chromospheric densities that can reach transition-region temperatures, then cool to less than $10^{4} \mathrm{~K}$ in minutes while remaining highly ionized. To reliably analyze such data requires a solid theoretical understanding of the annihilation process.

Positron production in solar flares has been intensively investigated (Ramaty et al. 1975; Kozlovsky et al. 1987, 2004) and the positrons were found to result from nuclear interactions of flare-accelerated particles with the solar atmosphere. The interactions produce radioactive isotopes and, if there are significant numbers of particles with energies of a few hundred $\mathrm{MeV}$, $\pi^{+}$mesons, all of which decay to produce positrons. The initial energies of the positrons depend on the specific emitter: several hundred $\mathrm{keV}$ for radioactive isotopes (e.g., positrons from ${ }^{11} \mathrm{C}$ and ${ }^{15} \mathrm{O}$ have $\beta$-decay spectra with endpoint energies of $\sim 0.97$ and $1.74 \mathrm{MeV}$, respectively) and from tens to hundreds of $\mathrm{MeV}$ for $\pi^{+}$(Murphy et al. 1987). In all cases, the initial positron energies are much higher than the thermal energies of the ambient particles. The positrons lose energy continuously via Coulomb collisions with any free electrons and discretely via excitation and ionization of any neutral atoms. (Radiation losses only become important at energies approaching $100 \mathrm{MeV}$.)

The positrons eventually annihilate with electrons, either directly or through the formation of a bound positronium atom (hereafter Ps). Direct annihilation of a positron-electron pair at rest results in the creation of two $\gamma$-ray photons, each of energy $\epsilon=m_{e} c^{2}=511 \mathrm{keV}$. If Ps is formed, the annihilation occurs from the ground state with the radiative outcome depending upon the total spin wave function of the Ps atom. There are four possible spin configurations of the ground state, one corresponding to total spin 0 (singlet) and three corresponding to total spin 1 (triplet) in units of $\hbar$. Since each occurs with equal probability, $1 / 4$ 
of the Ps atoms will be observed to be in the spin singlet state (parapositronium, hereafter ${ }^{1} \mathrm{Ps}$ ) and $3 / 4$ of the Ps atoms will be observed to be in the spin triplet state (orthopositronium, hereafter ${ }^{3} \mathrm{Ps}$ ). The singlet state has a lifetime $\tau_{1}=1.25 \times 10^{-10} \mathrm{~s}$ and its annihilation results in the creation of two $511 \mathrm{keV}$ photons $(2 \gamma)$ and the formation of a spectral line. The triplet state has a longer lifetime $\tau_{3}=1.42 \times 10^{-7} \mathrm{~s}$ and its annihilation yields three photons $(3 \gamma)$ with energies $\epsilon \leq 511 \mathrm{keV}$ and produces a continuum spectrum.

The only comprehensive theoretical study of positron annihilation in solar flares was done over 30 years ago in the pioneering work of Crannell et al. (1976). They identified for the first time the various annihilation processes relevant for solar flares. They calculated the $2 \gamma$ 511-ken line width and the flux ratio of the $3 \gamma$ continuum to the $2 \gamma$ line (hereafter, $\left.Q_{3 \gamma} / Q_{2 \gamma}\right)$ in H plasmas, assumed to be either neutral, completely ionized, or partially ionized (10\% and 50\%). Their calculations included depletion of ${ }^{3} \mathrm{Ps}$ (quenching) due to collisional interactions with neutral $\mathrm{H}$ (see discussion below).

Subsequent to this solar work, attention shifted to the transport and annihilation of positrons in the interstellar medium (ISM) following the balloon and satellite observations of a galactic annihilation line (see review by Lingenfelter \& Ramaty (1989)). In a seminal work, Bussard et al. (1979) (hereafter BRD) identified the processes and estimated the parameters relevant to the ISM. They also introduced the Monte-Carlo technique for calculating positron transport and Ps formation which has been used in all subsequent work. This interest in the Galactic annihilation line led to further studies of annihilation in tenuous media (e.g. Brown \& Leventhal (1987); Zurek et al. (1985); Guessoum et al. (1991); Wallyn et al. (1994); Chapuis et al. (1994); Guessoum et al. (2004)). Recently, Guessoum et al. (2005) presented updated calculations of positron annihilation in the ISM, including the effects of molecular H and grains. Churazov et al. (2005) also performed new calculations of positron annihilation in a low-density medium and applied them to SPI/INTEGRAL observations of the Galactic Center (Jean et al. 2003). Such calculations can be applied to solar flares, but the higher densities and temperatures often found in the solar environment require the inclusion of ${ }^{3}$ Ps quenching.

The complex network of processes associated with positron annihilation in solar flares is illustrated in Figure 1 and the various interactions are listed in Table 1. In flares, the four relevant annihilation processes are: (1) direct annihilation with free electrons (daf), (2) direct annihilation with bound electrons ( $d a b)$, (3) Ps formation via charge exchange with neutral atoms (ce), and (4) Ps formation via radiative combination with free electrons $(r c)$. As the positrons slow down from their initial energies, a fraction of them will form Ps by charge exchange with neutral atoms (process 3) before they have thermalized. (We note that while all four of the annihilation processes are possible before thermalization, only charge 
exchange is significant, as will be shown in §3.) When Ps is formed in this way, the positron velocity can be much greater than typical thermal velocities and the resulting annihilation radiation can be significantly Doppler broadened. The remaining positrons will thermalize and then annihilate via one of the four processes. In this case, the velocity distribution of the positron is Maxwellian, characterized by the temperature of the environment. Unless the temperature is quite high $\left(>10^{5} \mathrm{~K}\right)$, this annihilation radiation will be less Doppler broadened than that from annihilation via charge exchange before thermalization. The positron lifetime is therefore divided into two epochs: before thermalization (referred to as "in flight") and after thermalization.

Once Ps is formed, either by in-flight charge exchange (denoted 'ifce') or after thermalization, depletion of ${ }^{3}$ Ps before annihilation via collisional quenching can be significant when the density is sufficiently high, as in the deep layers of the solar atmosphere where positron production and subsequent annihilation can take place. (Because the lifetime of ${ }^{1} \mathrm{Ps}$ is a thousand times shorter than that of ${ }^{3} \mathrm{Ps}$, the required densities are a thousand times greater and so quenching is typically only relevant for ${ }^{3} \mathrm{Ps}$.) Quenching is caused by either ionization of ${ }^{3} \mathrm{Ps}$ or by conversion of ${ }^{3} \mathrm{Ps}$ to ${ }^{1} \mathrm{Ps}$ (spin flip). The density above which quenching becomes significant can be estimated. The cross section for the interaction is approximated by $\sigma=\pi a_{0}^{2}$ (where $a_{0}^{2}$ is the Bohr radius of the $\mathrm{H}$ atom) and the quenching rate is then $n_{\mathrm{H}} \sigma v$, where $n_{\mathrm{H}}$ is the total hydrogen number density and $v$ is the relative velocity, approximated by $\sqrt{k T / m_{e}}$. For ${ }^{3}$ Ps quenching to be important, this rate must be larger than the rate of annihilation from the triplet state $\left(1 / \tau_{3}\right)$, so that $n_{\mathrm{H}}>\left(\pi a_{0}^{2} \tau_{3} \sqrt{k T / m_{e}}\right)^{-1}$. At low densities and temperatures, such as those found in the ISM, such quenching processes can be neglected and the fraction of positrons which form $\mathrm{Ps}_{\mathrm{s}}\left(\mathrm{f}_{\mathrm{Ps}}\right)$ and $Q_{3 \gamma} / Q_{2 \gamma}$ are directly related by $Q_{3 \gamma} / Q_{2 \gamma}=2.25 \mathrm{f}_{\mathrm{Ps}} /\left(2-1.5 \mathrm{f}_{\mathrm{Ps}}\right)$.

Quenching cannot be neglected in the solar case. In a plasma of temperature $10^{4}$ $\mathrm{K}$, relevant to the solar atmosphere, the above estimate shows that quenching becomes important at densities $n_{\mathrm{H}} \simeq 10^{15} \mathrm{~cm}^{-3}$ which is typical of the chromosphere, and the direct relationship of $\mathrm{f}_{\mathrm{Ps}}$ and $Q_{3 \gamma} / Q_{2 \gamma}$ is broken. We will see that many of the quenching cross sections are, in fact, larger than $\pi a_{0}^{2}$ so the minimum density needed for quenching can be considerably less than this estimate. In addition, Ps formed in flight can have much higher velocities relative to the ambient medium which also reduces the density needed for quenching. Spin-flip quenching reduces the number of $3 \gamma$ continuum photons and increases the number of $2 \gamma$ line photons. Ionization quenching ejects positrons which can then form Ps again in flight before they thermalize.

From the above discussion, positron annihilation in solar flares is seen to be complex and the annihilation radiation is correspondingly rich in information on the temperature, density, 
and ionization state of the ambient medium where the positrons annihilate. Extracting the most useful information from annihilation radiation requires high spectral resolution measurements such as those obtained with RHESSI which, in turn, requires that the theory of annihilation in flares be updated. We report here on the results of such a effort with a focus on the understanding of the annihilation process after the positrons slow down to energies close to the thermal energy in the local plasma. Detailed treatment of the transport and energy loss of the high-energy positrons after they are produced will be the subject of a separate investigation.

In this work we follow the theoretical framework for positron annihilation developed by Crannell et al. (1976) and BRD but with significant improvements. Since those pioneering papers there has been an explosion of both laboratory measurements and calculations of cross sections for most of the processes involving positrons (see Charlton \& Humberston (2001)). We therefore update all cross-sections relevant for positron annihilation. We also extend the relevant reactions that were developed for $\mathrm{H}$ to include those for $\mathrm{He}$. Although $\mathrm{He} / \mathrm{H}$ in the Sun is only $\sim 10 \%$, there can be regions in the solar atmosphere where He remains neutral but $\mathrm{H}$ is fully ionized so that Ps formation via charge exchange with He can be an important process.

The calculations have been performed using computer algorithms that contain all the relevant physics associated with positron annihilation in solar flares. We first use an algorithm employing a Monte-Carlo technique to calculate (1) the fraction, $f_{1}$, of positrons that form Ps by in-flight charge exchange, (2) the resulting Ps energy distribution, and (3) the ${ }^{1}$ Ps $2 \gamma$ line shape. The remaining positrons thermalize, and we use several algorithms to calculate their interaction rates ( $d a f, d a b$, ce and $r c$ ) and their ${ }^{1} \mathrm{Ps}_{\mathrm{s}} 2 \gamma$ line shapes as functions of the ambient-medium temperature. We then use several algorithms to calculate the ${ }^{3} \mathrm{Ps}$ quenching rates as functions of the ambient-medium temperature for the ${ }^{3} \mathrm{Ps}$ energy distributions from each of the four Ps formation processes: rc, ce with $\mathrm{H}$, ce with $\mathrm{He}$, and ifce. For those positrons ejected by ionization quenching of ${ }^{3} \mathrm{Ps}$ formed in-flight, we reapply the Monte Carlo technique to calculate (1) the fraction, $f_{2}$, of these "second-generation" $(2 g)$ positrons that form Ps again before thermalization, (2) the resulting Ps energy distribution, (3) and the ${ }^{1}$ Ps $2 \gamma$ line shape. We also calculate the quenching rate of this second-generation Ps but we assume that any "third-generation" positrons ejected from ionization quenching will thermalize since most of them will have energies below the charge-exchange threshold of $6.8 \mathrm{eV}$. A final algorithm combines the results of all of the rate algorithms to calculate the relative contributions of each process and the final combined shape of the annihilation radiation spectrum. The algorithms are made available as a separate appendix to this paper.

In all of these calculations, we assume that the angular distribution of the positrons when 
they annihilate or form Ps is isotropic, which would certainly be true once the positrons have thermalized. Emission of positrons from radioactive emitters is isotropic in the emitter rest frame and relativistic effects will not change this distribution in the lab frame even if the accelerated particles were beamed because the recoil velocity of these nuclei after production is low. Also, in many cases the emitter lifetimes are long which will allow them time to slow down and isotropize. Positrons produced from pion decay, however, could be beamed if the accelerated particles were beamed. But since the only significant annihilation-related inflight interactions occur at relatively low energies after many interactions with free electrons or neutral atoms, the positron angular distribution should again be essentially isotropic. We ignore any possible focussing effects of transport of the positrons in the magnetic loop.

In $\S 2$ we describe in detail the most recent laboratory measurements and calculations of the various cross sections associated with positron annihilation: direct annihilation, Ps formation and quenching, and ionization and excitation of the ambient medium by positrons. In $\S 3$ we describe the Monte Carlo scheme we use to treat the in-flight interactions of positrons prior to thermalization. In $\S 4$ we calculate thermally-averaged rates as a function of temperature for each of the annihilation modes of thermalized positrons and for each of Ps quenching interactions using the updated cross sections. In $\S 5$ we describe the shape of the $2 \gamma$ annihilation line resulting from each of the annihilation modes and the $3 \gamma$ continuum. In $\S 6$ we use the rates calculated in $\S 4$ to solve a system of continuity equations for positron and Ps equilibrium which will provide the relative rates for each of the annihilation modes. In $\S 7$ we apply the results to four specific environments (fully ionized, completely neutral, partially ionized, and the solar atmosphere model C of Vernazza et al. (1981)), calculating the shape of the $2 \gamma$ line and the $Q_{3 \gamma} / Q_{2 \gamma}$ ratio for each. In $\S 8$ we apply the results to RHESSI data and in $\S 9$ we summarize.

\section{Cross Sections for Interactions of Positrons and Positronium}

In this section we present the most recent measurements and calculations of cross sections for the interactions relevant to positron annihilation in solar flares. Uncertainties of measured cross sections are typically less than 15\%. Uncertainties of calculated cross sections are estimated at less than $10 \%$ (Walters, priv. comm.). Where extrapolation to higher energies was required, the energy dependence is modeled on that of similar reactions of electrons (for which measurements generally exist) since the behavior of electrons and positrons at higher energies should be similar. Cross sections for direct annihilation of positrons with electrons are presented in $\S 2.1$, for Ps formation in $\S 2.2$, and for Ps quenching in $§ 2.3$. Positron energy-loss processes are discussed in $\S 2.4$. 


\subsection{Direct Annihilation}

In the plane-wave approximation, the direct annihilation of a positron and an electron with the emission of two photons $\left(e^{+} e^{-} \rightarrow 2 \gamma\right)$ has a total cross section in the center of mass given by (e.g. Heitler (1954))

$$
\sigma^{*}=\frac{\pi r_{0}^{2}}{4 \gamma^{* 2} \beta^{*}}\left[2\left(\beta^{* 2}-2\right)+\frac{3-\beta^{* 4}}{\beta^{*}} \ln \frac{1+\beta^{*}}{1-\beta^{*}}\right] .
$$

Here $\beta^{*} c$ is the magnitude of the velocity of either the electron or positron in the center of mass system, $\gamma^{*}=\left(1-\beta^{* 2}\right)^{-1 / 2}$, and $r_{0} \equiv e^{2} / m_{e} c^{2}=2.82 \times 10^{-13} \mathrm{~cm}$. (Hereafter, asterisks refer to the center of mass system.)

For the nonrelativistic region where most of the annihilations take place, this equation reduces to $\sigma^{*}=\pi r_{0}^{2} c / v$. At these nonrelativistic energies, the Coulomb attraction between the particles modifies the ingoing wave functions, requiring the inclusion of the factor $\left(2 \pi \alpha / \beta^{*}\right) /\left(1-e^{-2 \pi \alpha / \beta^{*}}\right)$ (see Crannell et al. (1976)), where the fine structure constant $\alpha \equiv e^{2} / \hbar c \simeq 1 / 137$. This cross section is shown in Figure 2 (multiplied by $10^{5}$ for plotting purposes). The cross section is much less than the cross sections for charge exchange (see $\S 2.2$ ), and rises with decreasing positron energy.

We do not explicitly show the cross section for direct annihilation with bound electrons. The calculation of the thermally-averaged rate for this process will be discussed in $\S 4$.

\subsection{Positronium Formation Processes}

Positrons can form Ps by charge exchange with atomic $\mathrm{H}$ or $\mathrm{He}\left(e^{+} \mathrm{H} \rightarrow \mathrm{H}^{+}\right.$Ps or $e^{+}$ $\left.\mathrm{He} \rightarrow \mathrm{He}^{+} \mathrm{Ps}\right)$ and by radiative combination with free electrons $\left(e^{+} e^{-} \rightarrow \gamma \mathrm{Ps}\right)$. Positronium formation through charge exchange with $\mathrm{H}$ has a threshold energy of $6.8 \mathrm{eV}$. In Figure 2 we

show the cross section measured by Zhou et al. (1997) from threshold to greater than 100 $\mathrm{eV}$ where it becomes negligible. Near the peak, this cross section is significantly less than that measured by Sperber et al. (1992) (used by Wallyn et al. (1994) and Guessoum et al. (1997)), but the Sperber measurement was later superseded by a new measurement by the same group (Weber et al. 1994) which is consistent with the Zhou et al. (1997) result.

The cross section for Ps formation through charge exchange with neutral He was measured from threshold $(17.8 \mathrm{eV})$ to $250 \mathrm{eV}$ by Overton et al. (1993) and is also shown in Figure 2. Above $100 \mathrm{eV}$, this cross section is less than that used by Chapuis et al. (1994) who used earlier measurements. 
We do not explicitly show the cross section for Ps formation via radiative combination with free electrons. The calculation of the rate for this process will be discussed in $\S 4$.

We neglect in-flight charge exchange with $\mathrm{He}^{+}$because (1) the cross section for this reaction (involving two positively-charged particles) is expected to be much smaller than that for neutral He, and (2) in an environment where there is significant $\mathrm{He}^{+}, \mathrm{H}$ would be be completely ionized and the increased positron energy loss due to free electrons would favor thermalization over charge exchange in flight with $\mathrm{He}^{+}$(and $\mathrm{He} / \mathrm{H}$ is only 0.1 ). We also neglect charge exchange of thermalized positrons with $\mathrm{He}^{+}$because $(1)$ of its significantly higher threshold energy $(47.6 \mathrm{eV})$ and $(2)$ the ionized environment would favor Ps formation by radiative combination with free electrons.

\subsection{Positronium Quenching Processes}

The cross sections for ionization and spin flip of ${ }^{3} \mathrm{Ps}$ by free electrons $\left(e^{-3} \mathrm{Ps} \rightarrow e^{-} e^{-} e^{+}\right.$ and $e^{-3} \mathrm{Ps} \rightarrow e^{-1} \mathrm{Ps}$ ) were recently calculated up to $80 \mathrm{eV}$ by Gilmore et al. (2004) and are shown in Figure 3. This is an important interaction in ionized gases at densities $>10^{13}$ $\mathrm{cm}^{-3}$, typical of the chromosphere. In their treatment of ${ }^{3}$ Ps quenching, Crannell et al. (1976) included only interactions with neutral $\mathrm{H}$ and hence underestimated the effects of quenching.

Ionization of ${ }^{3} \mathrm{Ps}$ by neutral $\mathrm{H}\left(\mathrm{H}^{3} \mathrm{Ps} \rightarrow \mathrm{H} e^{-} e^{+}\right)$was included in the analysis of Crannell et al. (1976) where the cross section calculated in the Born approximation by Massey \& Mohr (1954) was used. Here, we use more sophisticated calculations, using coupled pseudostate formalism, recently performed by Campbell et al. (1998b) up to $40 \mathrm{eV}$. Above that energy we use the calculations of Ray et al. (2002). The resulting cross section is shown in Figure 3. The cross section for spin flip of ${ }^{3} \mathrm{Ps}$ by $\mathrm{H}\left(\mathrm{H}^{3} \mathrm{Ps} \rightarrow \mathrm{H}^{1} \mathrm{Ps}\right)$ was recently calculated by Blackwood et al. (2002) and is also shown in Figure 3.

Much effort has gone into obtaining the cross section for the inverse process of charge exchange with ionized $\mathrm{H}\left(\mathrm{H}^{+} \mathrm{Ps} \rightarrow \mathrm{H} e^{+}\right)$since the charge conjugate reaction $\bar{p}$ Ps $\rightarrow$ $\overline{\mathrm{H}} e^{-}$results in the formation of anti-hydrogen. Because of time reversal invariance, the cross section for inverse charge exchange is related to that for charge exchange through (e.g. Humberston et al. (1987))

$$
\sigma\left(\mathrm{H}^{+} \mathrm{Ps} \rightarrow \mathrm{H} e^{+}\right)=\frac{E_{+}}{2 E_{\mathrm{Ps}}} \sigma\left(\mathrm{H} e^{+} \rightarrow \mathrm{H}^{+} \mathrm{Ps}\right)
$$

where $E_{+}$and $E_{\mathrm{Ps}}$ are the kinetic energies of the positron and Ps, respectively. Humberston et al. (1987) derived a cross section for this process from theoretical calculations of the 
charge exchange cross section. Here we use the measurements of the charge exchange cross section described above to obtain $\sigma\left(\mathrm{H}^{+} \mathrm{Ps} \rightarrow \mathrm{H} e^{+}\right)$. For inverse charge exchange in singlyionized $\mathrm{He}\left(\mathrm{He}^{+} \mathrm{Ps} \rightarrow \mathrm{He} e^{+}\right)$, we proceed as in the case for $\mathrm{H}$ and invert the He charge exchange cross section shown in Figure 2.

We neglect inverse charge exchange interactions with doubly-ionized He. The foremost reason for this is that if there is any appreciable ionization then free electrons will dominate the quenching. Also, the presence of doubly-ionized He would imply high temperature where direct annihilation (with free electrons) would dominate over Ps formation (by radiative combination). This, coupled with the fact that $\mathrm{He}$ is lower in abundance than $\mathrm{H}$ by an order of magnitude, justifies neglecting quenching by doubly-ionized He.

We neglect spin-flip and ionization quenching of Ps by neutral He because (1) if the medium is neutral, ionization by neutral $\mathrm{H}$ will dominate since $\mathrm{He} / \mathrm{H}=0.1$ and (2) if the medium is ionized, quenching by free electrons will dominate. In addition, spin-flip interactions resulting from electron exchange between Ps and neutral He have a high threshold of $19.8 \mathrm{eV}$. This high threshold results from the fact that in the ground state the electrons in the He atom must form a spin singlet and electron exchange interactions which convert ${ }^{3} \mathrm{Ps}$ to ${ }^{1} \mathrm{P}$ s require exciting He to the triplet state (Fraser \& Krady 1965). However, "pick-off" quenching can occur, where the Ps positron annihilates directly with a bound electron in which it shares a relative spin singlet state. The experimentally determined rate is pressure dependent (Fraser \& Krady 1965). Using the experimental results of Beers \& Hughs (1968) and the ideal gas law, the quenching rate is $7.3 \times 10^{-20} n T \mathrm{~s}^{-1}$ with $n$ in $\mathrm{cm}^{-3}$. For this to exceed the rate for annihilation requires $n T \gtrsim 10^{26} \mathrm{~K} \mathrm{~cm}^{-3}$ which is unlikely in the flare plasma.

We also neglect collisional spin flip due to interactions with ions because the cross sections are expected to be significantly lower than those for interactions where electrons are exchanged. Also, we do not include spin flip of ${ }^{3} \mathrm{Ps}$ due to magnetic fields. However, as has been measured by Pond \& Dicke (1952), there can be additional quenching by as much as $30 \%$ for magnetic field strengths of several thousand gauss.

\subsection{Positron Energy Loss}

Positrons can lose energy via discrete interactions with the neutral component of the plasma. The energy lost by the positron in these interactions is discussed in $§ 3.1$. Here we discuss the interaction cross sections. In Figure 2 we present the cross sections for the ionization reactions $e^{+} \mathrm{H} \rightarrow e^{+} e^{-} \mathrm{H}^{+}$and $e^{+} \mathrm{He} \rightarrow e^{+} e^{-} \mathrm{He}^{+}$. (These reactions are typically 
referred to as "impact" ionization to distinguish them from ionization associated with Ps formation through charge exchange, $e^{+} \mathrm{H} \rightarrow \mathrm{H}^{+} \mathrm{Ps}$ and $e^{+} \mathrm{He} \rightarrow \mathrm{He}^{+} \mathrm{Ps}$, which were treated in §2.2.) The cross section for impact ionization of $\mathrm{H}$ was measured by Jones et al. (1993) from threshold to $700 \mathrm{eV}$ and confirmed by Hoffman et al. (1997). These results are significantly lower than those of Spicher et al. (1990) which were used by Wallyn et al. (1994) in their study of annihilation in the ISM. The cross section for impact ionization of He was measured by Jacobsen et al. (1995) from threshold to $1500 \mathrm{eV}$. From threshold to $\sim 100 \mathrm{eV}$, these results are somewhat less than earlier published data (e.g., those of Fromme et al. (1986) used by Chapuis et al. (1994) who first included He reactions for the ISM).

There are no measurements of positron excitation of $\mathrm{H}$ (i.e., $e^{+} \mathrm{H} \rightarrow e^{+} \mathrm{H}^{*}$ ). However, the elaborate calculations of Kernoghan et al. (1996) successfully reproduced the available data for the total, elastic, ionization and positronium-formation cross sections. This success lends assurance that their calculation of the $1 \mathrm{~s}-2 \mathrm{~s}$ and $1 \mathrm{~s}-2 \mathrm{p}$ excitation cross sections are reliable as well. Stein et al. (1998) estimated the cross section for exciting the higher states of $\mathrm{H}$ by subtracting the partial cross sections for elastic, ionization, $1 \mathrm{~s}-2 \mathrm{~s}$ and $1 \mathrm{~s}-2 \mathrm{p}$ excitation, and Ps formation from the total cross section. In Figure 2 we show the resulting cross section for excitation to all levels of $\mathrm{H}$.

Mori et al. (1994) measured the total cross section for excitation of He by positrons $\left(e^{+} \mathrm{He} \rightarrow e^{+} \mathrm{He}^{*}\right)$ and it is shown in Figure 2. The recent calculation of Wu et al. (2004) is in general agreement with these data. We note that these results are significantly less than the cross section evaluated by Chapuis et al. (1994); their evaluation assumed that elastic scattering is negligible, as it is for H. But, as discussed by Campbell et al. (1998a), elastic scattering is significant for $\mathrm{He}$ and ionization is the largest cross section above $75 \mathrm{eV}$, in contrast to the result of Chapuis et al. (1994) in which excitation had the largest cross section.

We do not include excitation and ionization of $\mathrm{He}^{+}$because if $\mathrm{He}^{+}$is abundant, $\mathrm{H}$ will be mostly ionized and positron energy loss will be dominated by Coulomb interactions with free electrons.

Positrons can also lose energy continuously to the ionized component of the plasma. The energy-loss rate is given by the expression (Huba 1994)

$$
\frac{d E}{d t}=1.7 \times 10^{-8} n_{e} \beta^{-1} \ln \Lambda\left[\int_{0}^{E / k T} d x x^{1 / 2} e^{-x}-(E / k T)^{1 / 2} e^{-E / k T}\right] \quad(\mathrm{eV} / \mathrm{s})
$$

where $E$ and $\beta c$ are the positron energy $(\mathrm{eV})$ and velocity and $T$ and $n_{e}$ are the temperature 
and free electron density $\left(\mathrm{cm}^{-3}\right)$. The Coulomb logarithm is given by

$$
\ln \Lambda=\frac{\sqrt{\frac{k T}{4 \pi n_{e} e^{2}}}}{\max \left(\frac{2 e^{2}}{m u^{2}}, \frac{\hbar}{m u}\right)},
$$

with

$$
u=\sqrt{\frac{2 E}{m}}-\sqrt{\frac{8 k T}{\pi m}} .
$$

\section{In-Flight Positronium Formation}

When first produced, positrons have initial energies of $\sim 1 \mathrm{MeV}$ (from decay of radioactive positron emitters) to hundreds of $\mathrm{MeV}$ (from decay of pions). These positrons lose energy to the ambient medium via Coulomb interactions with free electrons and via ionization and excitation of neutral atoms. Coulomb interactions with free electrons will always dominate over direct annihilation or radiative combination with free electrons because these latter processes involve the emission of photons. The cross section for direct annihilation with bound electrons at high energies is simply the cross section for annihilation with free electrons (equation 1) which, at a few hundred $\mathrm{keV}$, is on the order of $10^{-26} \mathrm{~cm}^{2}$. Extrapolating the cross section for $\mathrm{H}$ ionization by positrons of Figure 2 to these energies as $\mathrm{E}^{-1}$ (Allen 1976) gives $10^{-21} \mathrm{~cm}^{2}$. Positrons at these energies will therefore lose energy via ionization rather than annihilating with free or bound electrons. The small fraction of positrons that do annihilate at higher energies will produce an annihilation line that will be significantly Doppler broadened and buried in the underlying continuum. The only remaining process is charge exchange whose cross section becomes significant at energies less than $\sim 100 \mathrm{eV}$ and dominates the other processes.

Positrons can therefore form Ps through charge exchange with neutral $\mathrm{H}$ and He as they slow down if the ambient medium is not fully ionized. At densities greater than $\sim 10^{13} \mathrm{~cm}^{-3}$, quenching via Ps ionization results in ejected positrons that can again form Ps in flight. We refer to these latter positrons as "second-generation" $(2 g)$ to distinguish them from the "primary" positrons. In $§ 3.1$ we discuss our calculations of in-flight Ps formation by primary positrons and in $\S 3.2$ we discuss in-flight Ps formation by second-generation positrons.

\subsection{Positronium Formation by Primary Positrons}

We use a Monte Carlo simulation to calculate the fraction of positrons that form Ps prior to thermalization via charge exchange $\left(f_{1}\right)$, the energy distribution of the resulting Ps, 
and the shape of the $2 \gamma$ line from the decay of ${ }^{1} \mathrm{Ps}$ in flight. The procedure is similar to that employed by BRD except that we include He and use the updated cross sections discussed above. In the simulation, positrons are injected into the plasma with an initial energy of $700 \mathrm{eV}$. The results are not sensitive to the exact initial energy as long as it is sufficiently above energies in the range $10-100 \mathrm{eV}$ where charge exchange is important. The positrons lose energy continuously to the ionized component of the plasma until they interact with a neutral $\mathrm{H}$ or He atom where they can excite, ionize or capture a bound electron to form Ps.

Given an initial energy $E_{0}$ and a uniformly-distributed random number $r$ from the unit interval, the energy $E$ at which the positron interacts discretely is determined from

$$
r=\exp \left[-c n_{\mathrm{H}} \int_{E_{0}}^{E} d E \frac{\beta \sigma_{\mathrm{tot}}}{d E / d t}\right],
$$

where $d E / d t$ is given by equation (3) and $\sigma_{t o t}$ is the total cross section for making any kind of discrete interaction with target $\mathrm{H}$ or He atoms, given by

$$
\sigma_{\text {tot }}=\left(1-X_{\mathrm{H}^{+}}\right)\left(\sigma_{\text {ex }}^{\mathrm{H}}+\sigma_{\text {ion }}^{\mathrm{H}}+\sigma_{c e}^{\mathrm{H}}\right)+\left(n_{\mathrm{He}} / n_{\mathrm{H}}\right)\left(1-X_{\mathrm{He}^{+}}-X_{\mathrm{He}^{++}}\right)\left(\sigma_{e x}^{\mathrm{He}}+\sigma_{i o n}^{\mathrm{He}}+\sigma_{c e}^{\mathrm{He}}\right) .
$$

where $X_{i}$ is the ratio of the number of ions of species $i$ to the total number of atoms of the given element (ionized plus neutral). The probability that a given type of discrete interaction occurs is then proportional to the cross section for that interaction. The cross sections were discussed in $\S 2$ and are shown in Figure 2.

The energy lost by the positron in interactions with neutral atoms is calculated as follows. As in BRD, in collisions which excite $\mathrm{H}$ the average amount of energy lost by the positron is taken to be $10.2 \mathrm{eV}$, the excitation energy of the $2^{1} \mathrm{~s}$ level. For ionization collisions with $\mathrm{H}$ the ejected electron velocity distribution is approximately Gaussian with a standard deviation of $0.382 \alpha c \simeq 2 \mathrm{eV}$ (where $\alpha$ is the fine structure constant) and centered at one fourth of the binding energy (BRD; Omidvar (1965)). Conservation of energy then provides the positron energy. For He we follow Chapuis et al. (1994) and adopt a mean energy loss per excitation of $20.6 \mathrm{eV}$, which corresponds to excitation from the ground state to the $2^{1} \mathrm{~s}$ level, and we use the value of $49 \mathrm{eV}$ (Opal et al. 1971) for the mean energy lost in an ionization collision.

After reducing the positron energy according to these prescriptions, the algorithm reinjects the positron and the above process is repeated until one of the three following outcomes occurs: (1) the energy of the positron falls below $6.8 \mathrm{eV}$, the threshold for charge exchange with $\mathrm{H}$; (2) the positron reaches thermal energies defined by $d E / d t>0$, i.e. the positron no longer loses energy; or (3) a charge-exchange interaction occurs. If either (1) or (2) happens then the positron is recorded as thermalizing. If (3) occurs then the charge exchange event 
is counted and the Ps energy is calculated, recorded and binned, where the energy of the Ps is the current energy of the positron less the difference between the ionization energy of $\mathrm{H}$ $(13.6 \mathrm{eV})$ or He $(24.6 \mathrm{eV})$ and the Ps binding energy $(6.8 \mathrm{eV})$. The entire process is then repeated until sufficiently good statistics are obtained for the Ps energy distribution.

In Figure 4 we show the fraction $f_{1}$ of positrons that form Ps in flight (solid curve) as a function of the ionization fraction of $\mathrm{H}, X_{\mathrm{H}^{+}}$. The temperature and electron density are fixed at $\mathrm{T}=11,600 \mathrm{~K}$ and $n_{e}=5 \times 10^{13} \mathrm{~cm}^{-3}$ and $\mathrm{He} / \mathrm{H}=0$, values assumed by BRD in their calculation of $f_{1}$. For a completely neutral medium we find $f_{1}=0.95$ which is the same as the value 0.95 found by BRD. The effect of inclusion of $\mathrm{He}(\mathrm{He} / \mathrm{H}=0.1)$ is shown by the dotted curve. The dot-dashed curve is discussed in $\S 3.2$.

Our results for $f_{1}$ are consistently higher than those of BRD (dashed line in Figure 4) for $X_{\mathrm{H}^{+}} \gtrsim 0.05$ even though our charge exchange cross section is very similar, our ionization and excitation cross sections are larger (which should reduce $f_{1}$ ), and we use an identical electron energy loss expression. To confirm our result, we have developed a second Monte Carlo code that directly follows positrons with initial energy of $700 \mathrm{eV}$ through small, finite path intervals $\Delta l$. For each interval we calculate the probabilities for each of the three discrete reactions $\left(n_{\mathrm{H}} \sigma_{i}\left(E_{+}\right) \Delta l\right.$ where $i$ is ce, ionization or excitation) at the current positron energy $\left(E_{+}\right)$. If charge exchange occurs, the event is counted. If ionization or excitation occurs, the energy is reduced according to the above prescriptions and the next interval is considered. If no discrete interaction occurs, the positron energy is reduced by $\Delta E=(d E / d l) \Delta l$ (where $\left.d E / d l=(d E / d t) v^{-1}\right)$ and the next interval is considered, unless the positron energy has fallen below the $6.8 \mathrm{eV}$ threshold or the positron has reached thermal energies defined by $d E / d t>0$. This process is repeated until reasonably good statistics are obtained. We find that our new code reproduces our original results for $f_{1}$ exactly. We have also compared our results with the recent calculations of positron annihilation in the interstellar medium by Guessoum et al. (2005) and find excellent agreement. We have no explanation for the differences with BRD, but we note that if we increase our $d E / d t$ by a factor 2 independent of energy, we reproduce the BRD results for $f_{1}$.

The solid histogram in Figure 5 shows the energy distribution of Ps formed in flight $\left(F_{\mathrm{Ps}}\right)$ in a neutral medium $(\mathrm{He} / \mathrm{H}=0.1)$. There is only a weak dependence of this distribution on temperature since most of the Ps is produced in the narrow range of energies between the threshold for charge exchange and excitation of $\mathrm{H}$ (the Ore gap (Charlton \& Humberston 2001); see Figure 2) where the only significant process is charge exchange. If the medium is only slightly ionized, Coulomb energy losses rapidly thermalize positrons just above the Ps formation threshold. This removes some of the lowest-energy Ps, slightly modifying the Ps formation energy distribution as shown by the dotted histogram in Figure 5 for $X_{\mathrm{H}^{+}}=0.05$ 
$\left(X_{\mathrm{He}^{+}}=X_{\mathrm{He}^{++}}=0\right)$.

From equation (3), the estimated energy-loss time $(E \div d E / d t)$ of a $30 \mathrm{eV}$ positron is $>10^{-6} \mathrm{~s}$ at an electron density of $10^{12} \mathrm{~cm}^{-3}$ (corresponding to an $\mathrm{H}$ density of $10^{14} \mathrm{~cm}^{-3}$ and $1 \%$ ionization fraction). Because Ps is both neutral and heavier than electrons, the energyloss time of $30 \mathrm{eV}$ Ps will be much longer than this and that of higher-energy Ps even longer. Since these times are longer than the lifetimes of either ${ }^{1} \mathrm{Ps}$ or ${ }^{3} \mathrm{Ps}$, Ps formed in-flight will not lose significant energy before annihilating or undergoing quenching. Therefore, both the Doppler-broadened shape of the $2 \gamma$ line from ${ }^{1}$ Ps decay and the ${ }^{3}$ Ps quenching rate will be determined by the energy distribution of the Ps at formation as calculated above. Because the velocities of Ps formed in-flight are larger than those for the thermal annihilation processes (see $\S 5$ ), the $2 \gamma$ line from ${ }^{1}$ Ps formed in flight will be correspondingly broader. Quenching will be discussed in $\S 4$.

\subsection{Positronium Formation by Second-Generation Positrons}

Because the energy distribution of the Ps at formation is unchanged before undergoing any quenching, positrons liberated via Ps ionization will still have energies significantly above thermal. As these second-generation positrons lose energy they may again form Ps via charge exchange before thermalization. We use the Monte Carlo simulation for Ps formation from primary positrons described above to also calculate the fraction of second-generation positrons that form Ps via charge exchange prior to thermalization $\left(f_{2}\right)$, the energy distribution of the resulting second-generation Ps, and the shape of the $2 \gamma$ line from the decay of ${ }^{1} \mathrm{Ps}$ in flight. In the simulation, a positronium energy is randomly selected from the distribution of Ps energies at the time of ionization $\left(F_{\mathrm{Ps}, \text { ion }}\right)$, an ejected positron energy is determined from this Ps energy, and the positron is injected with this initial energy into the above Monte Carlo. This is repeated until adequate counting statistics are achieved. The distribution $F_{\mathrm{Ps}, \text { ion }}$ is obtained from the proportionality:

$$
F_{\mathrm{Ps}, \text { ion }} \propto F_{\mathrm{Ps}} v \sigma_{3 \rightarrow 0}^{t o t},
$$

where $F_{\mathrm{Ps}}$ is the primary Ps energy distribution calculated above $(\S 3.1), v$ is the relative velocity (taken to be the velocity of the Ps since it is generally much higher than the thermal velocities of the ambient particles), and $\sigma_{3 \rightarrow 0}^{\text {tot }}$ is the total cross section for Ps breakup, given by

$$
\sigma_{3 \rightarrow 0}^{\text {tot }}=\left(1-X_{\mathrm{H}^{+}}\right) \sigma_{3 \rightarrow 0}^{\mathrm{H}}+\left(n_{e} / n_{\mathrm{H}}\right) \sigma_{3 \rightarrow 0}^{e}+X_{\mathrm{H}^{+}} \sigma_{i n v c e}^{\mathrm{H}}+\left(n_{\mathrm{He}} / n_{\mathrm{H}}\right) X_{\mathrm{He}^{+}} \sigma_{i n v c e}^{\mathrm{He}} .
$$

Here, $\sigma_{3 \rightarrow 0}^{\mathrm{H}}$ is the cross section for Ps ionization by neutral H, $\sigma_{3 \rightarrow 0}^{e}$ is the cross section for Ps ionization by free electrons, and $\sigma_{i n v c e}^{\mathrm{H}}$ and $\sigma_{i n v c e}^{\mathrm{He}}$ are the cross sections for inverse charge 
exchange by singly-ionized $\mathrm{H}$ and He, respectively. These quenching cross sections were discussed in $\S 2.3$.

We estimate the energy of the ejected positron in the following way. Because most of the energies of the primary in-flight Ps are above $10 \mathrm{eV}$ (see Figure 5), Ps breakup is dominated by ionization via either neutral $\mathrm{H}$ or free electrons (see Figure 3). For these reactions, there are three particles in the exit channel and the energy distribution of the ejected positron is expected to be distributed up to a maximum energy $E_{\max }$ with a peak at $E_{\max } / 2$, where $E_{\text {max }}=E_{\mathrm{Ps}}-6.8 \mathrm{eV}$ (the binding energy of Ps) and $E_{\mathrm{Ps}}$ is the energy of the Ps at the time of ionization. We approximate this positron energy distribution with a cosine function. We find that the shape of the resulting $2 \gamma$ line is insensitive to the exact distribution used.

The dot-dashed curve in Figure 4 shows the fraction $f_{2}$ of second-generation positrons that form Ps in flight as a function of $X_{\mathrm{H}^{+}}$, the ionization fraction of $\mathrm{H}$. As before, the temperature and electron density are fixed at $\mathrm{T}=11,600 \mathrm{~K}$ and $n_{e}=5 \times 10^{13} \mathrm{~cm}^{-3}$ and $\mathrm{He} / \mathrm{H}=0$. For a completely neutral medium we find $f_{2}=0.64$. The dashed histogram in Figure 5 shows the energy distribution of second-generation Ps formed in flight in a neutral medium $(\mathrm{He} / \mathrm{H}=0)$. Similar to primary Ps, there is only a weak dependence of this distribution on temperature since most of the Ps is produced in the Ore gap (see Figure 2 ). The $2 \gamma$ line from annihilation of ${ }^{1} \mathrm{Ps}$ produced by these second-generation positrons will be narrower than that from in-flight Ps formed from first-generation positrons because the second-generation Ps energy distribution is shifted to lower energies, as can be seen in Figure 5 (also see $\$ 5$ ). Positrons from ionization quenching of second-generation Ps are assumed to be thermalized since their energies will generally be below the charge exchange threshold.

\section{Interaction Rates and Positronium Energy Distributions}

In $\S 6$ we will develop relationships among the instantaneous numbers of thermalized positrons and singlet and triplet Ps atoms (formed via the various modes) in order to calculate the relative steady-state numbers of these species. These relationships will involve thermally-averaged rates for the interactions that thermalized positrons and Ps and in-flight Ps experience with the ambient thermal environment. For thermalized positrons, the rates are for direct annihilation (with both free and bound electrons) and Ps formation (via radiative combination with free electrons and Ps formation via charge exchange with $\mathrm{H}$ and He). For Ps, the rates are for the six Ps quenching reactions associated with each of the four Ps formation modes (radiative combination of thermal positrons with free electrons, charge exchange of thermal positrons with $\mathrm{H}$ and $\mathrm{He}$, and in-flight charge exchange). In this

section we calculate these rates and the energy distribution of the Ps associated with each 
the Ps-producing processes. (The energy distribution of Ps formed in flight was discussed in $\S 3$.$) The Ps energy distributions will be used in \S 5$ to calculate the associated ${ }^{1}$ Ps $2 \gamma$ line shapes. In the following calculations, we assume the ambient plasma is comprised of the following: $e^{-}$- free electrons; $\mathrm{H}$ - neutral hydrogen; $\mathrm{H}^{+}$- ionized hydrogen; $\mathrm{He}$ - neutral helium; $\mathrm{He}^{+}$- singly ionized helium; and $\mathrm{He}^{++}$- fully ionized helium.

The average rate per unit target density for a binary interaction in a plasma is defined by $\lambda \equiv\langle\sigma v\rangle$, where $\sigma$ is the total cross section for the given process and the relative velocity $v=\left|\mathbf{v}_{\mathbf{1}}-\mathbf{v}_{\mathbf{2}}\right|$, and $\mathbf{v}_{\mathbf{1}}$ and $\mathbf{v}_{\mathbf{2}}$ are the velocities of the interacting particles. The averaging is done over the velocity distributions of both interacting particles. For the ambient particle, this distribution is always Maxwellian characterized by the temperature. For reactions of thermalized positrons, the positron distribution is also Maxwellian characterized by the same temperature. In this case, the interaction rate (for $k T \ll m_{e} c^{2}$ ) is

$$
\lambda=\left(\frac{\sqrt{m_{1} m_{2}}}{2 \pi k T}\right)^{3} \int d^{3} \mathbf{v}_{\mathbf{1}} d^{3} \mathbf{v}_{\mathbf{2}} \exp \left(-\frac{m_{1} v_{1}^{2}}{2 k T}\right) \exp \left(-\frac{m_{2} v_{2}^{2}}{2 k T}\right) v \sigma\left(\mathbf{v}_{\mathbf{1}}, \mathbf{v}_{\mathbf{2}}\right) .
$$

For direct annihilation of thermal positrons with free electrons, equation (10) was numerically integrated by Crannell et al. (1976) and Bussard et al. (1979) using the cross section given in $\S 2.1$. The rate calculated by Bussard et al. (1979) is shown in Figure 6 as a function of temperature.

For direct annihilation of thermal positrons with bound electrons, the rate of reaction is often expressed in terms of the effective number of protons $\left(Z_{e f f}\right)$ multiplied by the free annihilation rate,

$$
\lambda=\pi r_{0}^{2} c Z_{\text {eff }} .
$$

$Z_{\text {eff }}$, which varies with the speed of the positron, is a measure of the probability of the positron being at the same position as one of the target electrons and is calculated from the elastic scattering wave function for the positron-target system. For $\mathrm{H}$, we use the rate given in BRD who used the thermally-averaged $Z_{\text {eff }}$ calculated by Bhatia et al. (1977). The resulting rate is shown in Figure 6. The curve stops at $10^{4} \mathrm{~K}$ since $\mathrm{H}$ will be mostly ionized above that temperature. For He, we integrate the $Z_{\text {eff }}$ given by Humberston (1974) over the positron Maxwellian distribution and the resulting rate is also shown in Figure 6.

For Ps formation via radiative combination of thermal positrons with free electrons we use the rate given by Gould (1989) with the Gaunt factor set equal to 0.9. This is also shown as a function of temperature in Figure 6 where it is seen that this process is more important than direct annihilation up to $T \simeq 7 \times 10^{5} \mathrm{~K}$; above this temperature, direct annihilation dominates. The energy distribution of the resulting Ps is Maxwellian (Gould 1989). The reason is that for $k T \ll m_{e} c^{2}$ the added velocity kick in the center of mass of 
the $e^{+} e^{-}$system due to the kinetic energy of the $e^{+} e^{-}$pair and the release of binding energy can be shown to go as $\beta_{k i c k} \simeq(6.8 \mathrm{eV}+k T) / 2 m_{e} c^{2}$, whereas the velocity of the center of mass system goes as $\beta_{c m} \simeq \sqrt{k T / m_{e} c^{2}}$, hence $\beta_{k i c k} \ll \beta_{c m}$.

For Ps formation via charge exchange of thermal positrons with $\mathrm{H}$ and $\mathrm{He}$, we integrated equation (10) using the cross sections given in $\S 2.2$ and the rates are also shown in Figure 6. The energy distribution of the resulting Ps is not Maxwellian and is derived as follows. Given a positron energy $E$, the conditional positronium energy distribution is

$$
F_{\mathrm{Ps}}\left(E_{\mathrm{Ps}} \mid E\right) \propto \sigma_{c e}(E) v(E) \delta\left(E_{\mathrm{Ps}}-(E-6.8 \mathrm{eV})\right)
$$

where we have assumed that the energy of the Ps is the initial energy of the positron less the difference between the ionization energy of $\mathrm{H}(13.6 \mathrm{eV})$ and the Ps binding energy (6.8 $\mathrm{eV})$. For He, $6.8 \mathrm{eV}$ is replaced by $17.8 \mathrm{eV}$ in equation (12) since the ionization energy of $\mathrm{He}$ is $24.6 \mathrm{eV}(24.6-6.8=17.8)$. Integrating equation $(12)$ over the positron Maxwellian velocity distribution gives the Ps energy distributions for $\mathrm{H}$ and $\mathrm{He}$ :

$$
\begin{aligned}
& F_{P s}(E)=\frac{1}{N_{\mathrm{Ps}}} \frac{d N_{\mathrm{Ps}}}{d E}(E) \propto \sigma_{c e}^{\mathrm{H}}(E+6.8 \mathrm{eV}) v(E+6.8 \mathrm{eV}) \sqrt{E+6.8 \mathrm{eV}} \exp [-(E+6.8 \mathrm{eV}) / k T] \\
& F_{P s}(E)=\frac{1}{N_{\mathrm{Ps}}} \frac{d N_{\mathrm{Ps}}}{d E}(E) \propto \sigma_{c e}^{\mathrm{He}}(E+17.8 \mathrm{eV}) v(E+17.8 \mathrm{eV}) \sqrt{E+17.8 \mathrm{eV}} \exp [-(E+17.8 \mathrm{eV}) / k T] .
\end{aligned}
$$

We now calculate the six ${ }^{3} \mathrm{Ps}$ quenching rates for each of the four Ps production processes (radiative combination of thermal positrons with free electrons, charge exchange of thermal positrons with $\mathrm{H}$ and $\mathrm{He}$, and charge exchange in flight). The six quenching cross sections were discussed in $\S 2.3$ and are shown in Figure 3. For each of the four Ps-formation cases, the velocity distribution of the ambient particle (electron, $\mathrm{H}, \mathrm{H}^{+}$or $\mathrm{He}^{+}$) is Maxwellian but the velocity distribution of the Ps depends on the formation process as discussed above. For Ps formed via radiative combination of thermal positrons with free electrons, the Ps distribution is also Maxwellian and equation (10) may be used. The resulting rates are shown in panel (a) of Figure 7. For Ps formed via charge exchange of thermal positrons with ambient $\mathrm{H}$ and $\mathrm{He}$, the Ps velocity distribution is given by equations (13) and (14) for $\mathrm{H}$ and $\mathrm{He}$, respectively. Averaging these distributions with the Maxwellian ambient-particle distributions results in the quenching rates shown in panels (b) and (c) of Figure 7. For charge exchange in flight, the primary Ps distribution is similar to that shown in Figure 5 and the resulting quenching rates for Ps formed from primary positrons are shown in panel $\mathrm{d}$ of Figure 7. We note that all of these in-flight quenching rates are nearly independent of temperature. This is because the velocity of the Ps (energies of tens of eV, Figure 5) is 
much higher than the thermal velocities of the ambient particles so that the relative velocity is essentially the velocity of the Ps. The relative velocity (and therefore the rate) is then independent of temperature. Also, the calculations for the in-flight rates are only performed up to $2 \times 10^{5} \mathrm{~K}$. Above this temperature there will probably not be sufficient numbers of neutral atoms for charge exchange to be important.

\section{Annihilation Radiation Spectra}

The shape and corresponding width of the positron annihilation line is different for each of the modes of annihilation shown in Figure 1: direct with free and bound electrons and via Ps formed by radiative combination and by charge exchange (either after thermalization or in flight). The shape of the observed total annihilation radiation spectrum (both $2 \gamma$ line and $3 \gamma$ continuum) is a composite of these components and their relative contributions depend on the temperature, density, ionization and composition of the ambient medium (as will be discussed in $\S 6$ ). In this section we discuss the shapes of each of the various components: the $2 \gamma$ line is discussed in $\S 5.1$ and the $3 \gamma$ continuum in $§ 5.2$.

\subsection{The Annihilation Line}

The shape of the $2 \gamma$ line resulting from the direct annihilation of nonrelativistic thermal

positrons and free electrons is Gaussian with a full-width at half maximum (FWHM) given by

$$
\mathrm{FWHM}=m c^{2} \sqrt{\ln 2\left(\frac{k T}{m c^{2}}\right)}=1.1 \mathrm{keV} \sqrt{\frac{T}{10^{4} \mathrm{~K}}} .
$$

as derived in Appendix A. Since the centers of the positron and electron energy distributions in the center of mass frame are on the order of $k T$, the line center will be blue-shifted to $\epsilon=m_{e} c^{2}+\phi k T$ where $\phi$ is on the order of unity (Gould 1989). In the Sun, the annihilation line could therefore be blue shifted by $\sim 1 \mathrm{eV}$.

Similarly, the shape of the annihilation line resulting from the $2 \gamma$ decay of ${ }^{1} \mathrm{Ps}$ formed via radiative combination of thermal positrons with free electrons also has a width given by equation (15). As shown above $(\S 4)$, Ps formed in this way has a Maxwellian velocity distribution. Since the photon is emitted isotropically in the rest frame of the Ps and has energy in the lab frame given by equation (A3), the photon is distributed in energy according to equation (A10) from which the width given by equation (15) is obtained (see also an alternative derivation by Gould (1989)). 
However, the velocity distribution of Ps formed through thermal charge exchange is not Maxwellian and the shape of the $2 \gamma$ line from ${ }^{1}$ Ps decay is not Gaussian. To derive the line shape for this decay $\left({ }^{1} \mathrm{Ps} \rightarrow 2 \gamma\right)$, we follow the derivation by Stecker (1971) for the analogous decay of $\pi_{0}\left(\pi_{0} \rightarrow 2 \gamma\right)$. The conditional photon energy distribution for a given Ps energy $E_{\mathrm{Ps}}$ is flat and, normalized, is given by

$$
\begin{aligned}
F_{\gamma}\left(\epsilon \mid E_{\mathrm{Ps}}\right) & =\frac{1}{2 m_{e} c^{2} \gamma_{\mathrm{Ps}} \beta_{\mathrm{Ps}}} \\
& =\frac{1}{\sqrt{E_{\mathrm{Ps}}\left(E_{\mathrm{Ps}}+4 m_{e} c^{2}\right)}},
\end{aligned}
$$

where the photon energy $\epsilon$ is between the Doppler-shift limits

$$
\gamma_{\mathrm{Ps}}\left(1-\beta_{\mathrm{Ps}}\right) m_{e} c^{2}<\epsilon<\gamma_{\mathrm{Ps}}\left(1+\beta_{\mathrm{Ps}}\right) m_{e} c^{2}
$$

and vanishes outside this range. Using the conditional Ps energy distribution for a positron of energy $E\left(F_{\mathrm{Ps}}\left(E_{\mathrm{Ps}} \mid E\right)\right.$; equation 12 in $\left.\S 4\right)$ and integrating over the Maxwellian distribution of the positron, the photon energy distribution for $\mathrm{H}$ is then

$$
\begin{aligned}
f_{\gamma}(\epsilon) & \propto \int d E_{\mathrm{Ps}} F_{\gamma}\left(\epsilon \mid E_{\mathrm{Ps}}\right) \int d E F_{\mathrm{Ps}}\left(E_{\mathrm{Ps}} \mid E\right) \sqrt{E} e^{-E / k T} \\
& \propto \int_{6.8 \mathrm{eV}+\left(\epsilon-m_{e} c^{2}\right)^{2} / \epsilon}^{\infty} d E \frac{\sqrt{E} e^{-E / k T} \sigma_{c e}(E) v(E)}{\sqrt{(E-6.8 \mathrm{eV})\left(E-6.8 \mathrm{eV}+4 m_{e} c^{2}\right)}} .
\end{aligned}
$$

The lower limit of integration is the minimum energy the Ps needs to produce a photon of energy $\epsilon$ (Stecker 1971). For He, $6.8 \mathrm{eV}$ is replaced by $17.8 \mathrm{eV}$. In Figure 8 we show the resulting line shapes for thermal charge exchange in $\mathrm{H}$ (solid curves) and He (dashed curves) for various temperatures.

As shown in $\S 3$, the energy distributions of Ps formed in flight from primary positrons and from second-generation positrons resulting from ionization of primary in-flight Ps are also not Maxwellian (see Figure 5). From our Monte Carlo simulations we find that the shapes of the associated annihilation lines are not Gaussian. The solid and dashed curves in Figure 9 show the line shapes from primary and second-generation Ps, respectively, in a neutral medium $(\mathrm{He} / \mathrm{H}=0.1)$. While these shapes are not quite Gaussian, we will characterize their widths by a formal full-width at half maximum. The FWHM of the primary line is $\sim 6.1 \mathrm{keV}$. (We obtained 5.9 and $7.1 \mathrm{keV}$ FWHM for pure $\mathrm{H}$ and He, respectively. The value of 5.9 is somewhat less than the value of $6.4 \mathrm{keV}$ obtained by BRD for pure H.) If the medium is only slightly ionized, positrons just above the Ps formation threshold are rapidly thermalized, slightly modifying the Ps formation energy distribution (see $\S 3$ and dotted histogram of 
Figure 5). As a result, there are fewer photons with energies very near $511 \mathrm{keV}$, flattening the line profile as shown by the dotted distribution in Figure 9 . At $\sim 6.9 \mathrm{keV}$, the FWHM is slightly larger than the neutral case. The FWHM of the second-generation line is $\sim 2.6 \mathrm{keV}$ (in a neutral medium with $\mathrm{He} / \mathrm{H}=0.1$ ), narrower than the first-generation line, reflecting the shift to lower energies of the Ps energy distribution (see Figure 5). These results are only weakly dependent on density and temperature (see $\S 3$ ).

To obtain the shape of the line produced by direct annihilation of thermal positrons with bound electrons it is necessary to know the momentum distribution of the atomic electrons. We first treat the case where the energies of the positrons are near zero. In this case, the shape of the line is due mostly to the momentum distribution of the bound electron. For H, this distribution was calculated by Humberston \& Wallace (1972) and for He by Humberston (1974) and Van Reeth et al. (1996). These calculations showed that the shape of the annihilation line is nearly Gaussian with a FWHM width of $1.4 \mathrm{keV}$ for $\mathrm{H}$ and 2.5 $\mathrm{keV}$ for He. These results have been confirmed by laboratory measurements (Van Reeth et al. 1996).

At temperatures greater than $\sim 3 \times 10^{4} \mathrm{~K}$ for $\mathrm{H}$ and $\sim 1 \times 10^{5} \mathrm{~K}$ for He, the Maxwellian momentum distribution of the thermal positrons begins to affect the shape of the line. At higher temperatures, the effect of the thermal positron dominates, and the shape of the line is Gaussian. The shape of the combined line for all temperatures can then be approximated by a Gaussian with a FWHM $(\Gamma)$ given by

$$
\Gamma^{2}=\Gamma_{\mathrm{e}^{-}}^{2}+\Gamma_{\mathrm{e}^{+}}^{2}
$$

where $\Gamma_{\mathrm{e}^{+}}$and $\Gamma_{\mathrm{e}^{-}}$are the widths for the thermal positron and bound electron components, respectively, with $\Gamma_{\mathrm{e}^{-}}=1.4 \mathrm{keV}$ for $\mathrm{H}$ and $2.5 \mathrm{keV}$ for $\mathrm{He}$ and

$$
\Gamma_{\mathrm{e}^{+}}=m c^{2} \sqrt{2 \ln 2\left(\frac{k T}{m c^{2}}\right)},
$$

derived similarly to the derivation given in Appendix A for the shape of the line from direct annihilation of positrons and free electrons.

We summarize the various shapes and widths of the annihilation line from the five modes of annihilation.

1. Direct annihilation of thermal positrons with free electrons. The shape of the line is Gaussian with the FWHM given by equation (15). The annihilation rate by this mode is shown in Figure 6.

2. Radiative combination of thermal positrons with free electrons. The shape 
of the line from the ${ }^{1}$ Ps decay is Gaussian with the FWHM given by equation (15). The Ps formation rate by this mode is shown in Figure 6 .

3. Positronium formation in flight. The shape of the line from the ${ }^{1}$ Ps decay is not Gaussian (see Figure 9) and has a FWHM of $\sim 6.1 \mathrm{keV}$ for Ps from primary positrons and $\sim 2.6 \mathrm{keV}$ for Ps from second-generation positrons. The fractions of primary and secondgeneration positrons forming Ps in flight are shown in Figure 4.

4. Charge exchange of thermal positrons with atoms. The shape of the line from the ${ }^{1}$ Ps decay is not Gaussian. It is given by equation (18) and is shown in Figure 8. The Ps formation rate by this mode is shown in Figure 6.

5. Direct Annihilation of Thermal Positrons with Bound Electrons. The shape is approximately Gaussian with the FWHM given by equation (19). The annihilation rate by this mode is shown in Figure 6 .

\subsection{Positronium Continuum}

The decay of ${ }^{3}$ Ps results in three photons which, in the rest frame of the Ps, are distributed in energy according to (Ore \& Powell 1949)

$$
\begin{aligned}
\frac{1}{N_{3 \gamma}} \frac{d N_{3 \gamma}}{d \epsilon}(\epsilon)= & \frac{2}{\left(\pi^{2}-9\right) m_{e} c^{2}}\left[\frac{\epsilon\left(m_{e} c^{2}-\epsilon\right)}{\left(2 m_{e} c^{2}-\epsilon\right)^{2}}+\frac{2 m_{e} c^{2}\left(m_{e} c^{2}-\epsilon\right)}{\epsilon^{2}} \ln \left(\frac{m_{e} c^{2}-\epsilon}{m_{e} c^{2}}\right)\right. \\
& \left.-\frac{2 m_{e} c^{2}\left(m_{e} c^{2}-\epsilon\right)^{2}}{\left(2 m_{e} c^{2}-\epsilon\right)^{3}} \ln \left(\frac{m_{e} c^{2}-\epsilon}{m_{e} c^{2}}\right)+\frac{\left(2 m_{e} c^{2}-\epsilon\right)}{\epsilon}\right] .
\end{aligned}
$$

\section{Positronium and Thermal Positron Equilibrium in a Plasma and the Total Annihilation Radiation Spectrum}

As shown in $\S 5$, the shape of the $2 \gamma$ line can be different for each of the annihilation modes. The total annihilation radiation spectrum (both $2 \gamma$ line and $3 \gamma$ continuum) is composed of contributions from each of the modes. In order to construct this total spectrum, we need to know the relative strengths of each mode for a given combination of temperature, density and ionization state of the environment. These strengths can be determined relatively easily if the system is in a steady state condition. It will be in steady state if the time scale over which positrons slow down and annihilate is much shorter than the time scale over which they are produced.

The time history for positron production is obtained by convolving the time history 
for production of the positron emitters with their exponential decays. The decay times of the most important positron emitters are on the order of a few minutes (Kozlovsky et al. 1987). The time required for positrons to slow down and annihilate can be estimated from comparisons of calculated positron production time histories with measured $511 \mathrm{keV}$ annihilation-line time histories from solar flares. Since no appreciable differences were found (Murphy et al. 1984), the slowing-down times must be shorter than the temporal resolution of the instrument (16 seconds for $S M M$ ) which is shorter than the positron production time scales. The assumption of steady state is therefore valid.

In $\S 6.1$ we develop a system of conservation equations and solve it for the numbers of positrons and ${ }^{1} \mathrm{Ps}$ and ${ }^{3} \mathrm{Ps}$ atoms, assuming steady state. In $\S 6.2$, we use the relative numbers to construct the total annihilation radiation spectrum.

\subsection{System of Continuity Equations}

We write a network of continuity equations for the conservation of the number densities of thermal positrons and Ps formed via the various modes and, assuming steady state, set all of their partial derivatives with respect to time equal to zero. The $2 \gamma$ and $3 \gamma$ production rates, and hence the emergent spectrum, can then be obtained from the resulting number densities which all scale linearly with the positron injection rate per unit volume $Q_{+}$.

The network of reactions was displayed graphically in Figure 1 and particle conservation is expressed through the following system of continuity equations:

$$
\begin{aligned}
\frac{\partial N_{3}^{i f c e}}{\partial t} & =\frac{3}{4} f_{1} Q_{+}-N_{3}^{i f c e} R_{3 \rightarrow x}^{i f c e}-\frac{N_{3}^{i f c e}}{\tau_{3}}=0 \\
\frac{\partial N_{3}^{2 g}}{\partial t} & =\frac{3}{4} f_{2} N_{3}^{i f c e} R_{3 \rightarrow 0}^{i f c e}-N_{3}^{2 g} R_{3 \rightarrow x}^{2 g}-\frac{N_{3}^{2 g}}{\tau_{3}}=0 \\
\frac{\partial N_{3}^{H c e}}{\partial t} & =\frac{3}{4} N_{+} R^{H c e}-N_{3}^{H c e} R_{3 \rightarrow x}^{H c e}-\frac{N_{3}^{H c e}}{\tau_{3}}=0 \\
\frac{\partial N_{3}^{H e c e}}{\partial t} & =\frac{3}{4} N_{+} R^{H e c e}-N_{3}^{H e c e} R_{3 \rightarrow x}^{H e c e}-\frac{N_{3}^{H e c e}}{\tau_{3}}=0 \\
\frac{\partial N_{3}^{r c}}{\partial t} & =\frac{3}{4} N_{+} R^{r c}-N_{3}^{r c} R_{3 \rightarrow x}^{r c}-\frac{N_{3}^{r c}}{\tau_{3}}=0 \\
\frac{\partial N_{1}^{i f c e}}{\partial t} & =\frac{1}{4} f_{1} Q_{+}+N_{3}^{i f c e} R_{3 \rightarrow 1}^{i f c e}-\frac{N_{1}^{i f c e}}{\tau_{1}}=0 \\
\frac{\partial N_{1}^{2 g}}{\partial t} & =\frac{1}{4} f_{2} N_{3}^{i f c e} R_{3 \rightarrow 0}^{i f c e}+N_{3}^{2 g} R_{3 \rightarrow 1}^{2 g}-\frac{N_{1}^{2 g}}{\tau_{1}}=0
\end{aligned}
$$




$$
\begin{aligned}
\frac{\partial N_{1}^{H c e}}{\partial t}= & \frac{1}{4} N_{+} R^{\text {Hce }}+N_{3}^{\text {Hce }} R_{3 \rightarrow 1}^{\text {Hce }}-\frac{N_{1}^{\text {Hce }}}{\tau_{1}}=0 \\
\frac{\partial N_{1}^{H e c e}}{\partial t}= & \frac{1}{4} N_{+} R^{\text {Hece }}+N_{3}^{\text {Hece }} R_{3 \rightarrow 1}^{\text {Hece }}-\frac{N_{1}^{\text {Hece }}}{\tau_{1}}=0 \\
\frac{\partial N_{1}^{r c}}{\partial t}= & \frac{1}{4} N_{+} R^{r c}+N_{3}^{r c} R_{3 \rightarrow 1}^{r c}-\frac{N_{1}^{r c}}{\tau_{1}}=0 \\
\frac{\partial N_{+}}{\partial t}= & \left(1-f_{1}\right) Q_{+}+\left(1-f_{2}\right) N_{3}^{i f c e} R_{3 \rightarrow 0}^{\text {ifce }}+N_{3}^{2 g} R_{3 \rightarrow 0}^{2 g} \\
& +N_{3}^{\text {Hce }} R_{3 \rightarrow 0}^{\text {Hce }}+N_{3}^{\text {Hece }} R_{3 \rightarrow 0}^{\text {Hece }}+N_{3}^{r c} R_{3 \rightarrow 0}^{r c} \\
& -N_{+}\left(R^{\text {Hce }}+R^{\text {Hece }}+R^{r c}+R^{\text {Hdab }}+R^{\text {Hedab }}+R^{\text {daf }}\right)=0 .
\end{aligned}
$$

Here $N_{+}$is the number of thermal positrons. $N_{1}^{i}$ and $N_{3}^{i}$ are the numbers of Ps atoms in the singlet and triplet states, with the superscript $i$ referring to the Ps formation process: Hcecharge exchange with $\mathrm{H}$; Hece - charge exchange with He; rc - radiative combination with free electrons; if ce - first-generation in-flight charge exchange; and $2 g$ - second-generation in-flight charge exchange; each of which has its own Ps velocity distribution. Rates for Ps formation processes (in units of $s^{-1}$ ) are denoted $R^{i}$ where the superscript $i$ has the same meaning as above. These rates are the rates calculated above (the $\lambda$ 's, see $\S 4$ ) multiplied by the relevant target densities. Direct annihilation rates are also denoted $R^{i}$, but with the superscript $i$ referring to the annihilation channel: $H d a b$ - direct annihilation with electrons bound in $\mathrm{H}$; Hedab - direct annihilation with electrons bound in He; and daf - direct annihilation with free electrons. Triplet Ps quenching rates are denoted $R_{3 \rightarrow 0}^{i}$ for tripletstate ionization, $R_{3 \rightarrow 1}^{i}$ for triplet-singlet spin flip, and $R_{3 \rightarrow x}^{i}=R_{3 \rightarrow 0}^{i}+R_{3 \rightarrow 1}^{i}$, where again the superscript $i$ has the same meaning as above.

This system of equations can then be solved for the particle numbers . First,

$$
N_{+}=Q_{+}\left[\frac{\left(1-f_{1}\right)+\left(\frac{\frac{3}{4} f_{1} R_{3 \rightarrow 0}^{i f c e}}{R_{3 \rightarrow x}^{i f c e}+\frac{1}{\tau_{3}}}\right)\left(\left(1-f_{2}\right)+\left(\frac{\frac{3}{4} f_{2} R_{3 \rightarrow 0}^{2 g}}{R_{3 \rightarrow x}^{2 g}+\frac{1}{\tau_{3}}}\right)\right)}{\sum_{i} R^{i}-\sum_{j}\left(\frac{\frac{3}{4} R^{j} R_{3 \rightarrow 0}^{j}}{R_{3 \rightarrow x}^{j}+\frac{1}{\tau_{3}}}\right)}\right],
$$

where $i=\{H c e, H e c e, r c, H d a b, H e d a b, d a f\}$ and $j=\{H c e, H e c e, r c\}$. The rest of the particle numbers can be expressed in terms of $Q_{+}$and $N_{+}$. We have

$$
\begin{aligned}
N_{3}^{i f c e} & =\frac{\frac{3}{4} f_{1} Q_{+}}{R_{3 \rightarrow x}^{i f c e}+\frac{1}{\tau_{3}}} \\
N_{3}^{2 g} & =\frac{3}{4} f_{2} N_{3}^{i f c e}\left[\frac{R_{3 \rightarrow 0}^{i f c e}}{R_{3 \rightarrow x}^{2 g}+\frac{1}{\tau_{3}}}\right]
\end{aligned}
$$




$$
\begin{aligned}
N_{3}^{H c e} & =\frac{\frac{3}{4} R^{H c e} N_{+}}{R_{3 \rightarrow x}^{H c e}+\frac{1}{\tau_{3}}}, \\
N_{3}^{\text {Hece }} & =\frac{\frac{3}{4} R^{H e c e} N_{+}}{R_{3 \rightarrow x}^{H e c e}+\frac{1}{\tau_{3}}}, \\
N_{3}^{r c} & =\frac{\frac{3}{4} R^{r c} N_{+}}{R_{3 \rightarrow x}^{r c}+\frac{1}{\tau_{3}}}, \\
N_{1}^{i f c e} & =\frac{1}{4} \tau_{1} f_{1} Q_{+}\left[\frac{R_{3 \rightarrow x}^{i f c e}+\frac{1}{\tau_{3}}+3 R_{3 \rightarrow 1}^{i f c e}}{R_{3 \rightarrow x}^{i f c e}+\frac{1}{\tau_{3}}}\right], \\
N_{1}^{2 g} & =\frac{1}{4} \tau_{1} f_{2} N_{3}^{i f c e} R_{3 \rightarrow 0}^{i f c e}\left[\frac{R_{3 \rightarrow x}^{2 g}+\frac{1}{\tau_{3}}+3 R_{3 \rightarrow 1}^{2 g}}{R_{3 \rightarrow x}^{2 g}+\frac{1}{\tau_{3}}}\right], \\
N_{1}^{H c e} & =\frac{1}{4} \tau_{1} R^{H c e} N_{+}\left[\frac{R_{3 \rightarrow x}^{H c e}+\frac{1}{\tau_{3}}+3 R_{3 \rightarrow 1}^{H c e}}{R_{3 \rightarrow x}^{H c e}+\frac{1}{\tau_{3}}}\right], \\
N_{1}^{H e c e} & =\frac{1}{4} \tau_{1} R^{H e c e} N_{+}\left[\frac{R_{3 \rightarrow x}^{H e c e}+\frac{1}{\tau_{3}}+3 R_{3 \rightarrow 1}^{H c e}}{R_{3 \rightarrow x}^{H e c e}+\frac{1}{\tau_{3}}}\right], \\
N_{1}^{r c} & =\frac{1}{4} \tau_{1} R^{r c} N_{+}\left[\frac{R_{3 \rightarrow x}^{r c}+\frac{1}{\tau_{3}}+3 R_{3 \rightarrow 1}^{r c}}{R_{3 \rightarrow x}^{r c}+\frac{1}{\tau_{3}}}\right] .
\end{aligned}
$$

These particle numbers depend on the rates $R$ which, in turn, depend on the density, temperature and ionization state of the environment.

From the particle numbers we can determine the rate for producing $2 \gamma$ line photons,

$$
Q_{2 \gamma}=2 N_{+}\left(R^{H d a b}+R^{H e d a b}+R^{d a f}\right)+\frac{2}{\tau_{1}}\left(N_{1}^{i f c e}+N_{1}^{H c e}+N_{1}^{H e c e}+N_{1}^{r c}+N_{1}^{2 g}\right),
$$

and $3 \gamma$ continuum photons,

$$
Q_{3 \gamma}=\frac{3}{\tau_{3}}\left(N_{3}^{i f c e}+N_{3}^{H c e}+N_{3}^{H e c e}+N_{3}^{r c}+N_{3}^{2 g}\right)
$$

These rates are directly proportional to $Q_{+}$and the ratio $Q_{3 \gamma} / Q_{2 \gamma}$ is therefore independent of $Q_{+}$.

\subsection{Composite Annihilation Radiation Spectrum}

The composite $2 \gamma$ annihilation line is a combination of the line shapes from each of the annihilation modes (see $§ 5.1$ ). The relative strength of each mode is given by the individual 
terms of equation (25) as calculated for the temperature, density and ionization state of the environment. The composite line shape can be very different from the shape from any one of the modes.

Let $G\left(\epsilon \mid \epsilon_{0}, \Gamma\right)$ denote a normalized Gaussian centered at $\epsilon_{0}$ with FWHM denoted by $\Gamma$. Similarly, let $H\left(\epsilon \mid \epsilon_{0}, \Gamma\right)$ denote the line shape resulting from thermal charge exchange and $J^{i f c e}\left(\epsilon \mid \epsilon_{0}, \Gamma\right)$ and $J^{2 g}\left(\epsilon \mid \epsilon_{0}, \Gamma\right)$ denote the line shapes resulting from primary and secondgeneration in-flight charge exchange, respectively (see §5.1). (We note that these latter shapes can be far from Gaussian and the formal FWHM may not adequately reflect this shape.) Then the composite $2 \gamma$ line shape is given by

$$
\begin{aligned}
\frac{d Q_{2 \gamma}}{d \epsilon}(\epsilon)=2 N_{+} & {\left[R^{\text {Hdab }} G\left(\epsilon \mid m_{e} c^{2}, \Gamma_{\text {Hdab }}\right)\right.} \\
& +R^{\text {Hedab }} G\left(\epsilon \mid m_{e} c^{2}, \Gamma_{\text {Hedab }}\right) \\
& \left.+R^{\text {daf }} G\left(\epsilon \mid m_{e} c^{2}, \Gamma_{d a f}\right)\right] \\
+\frac{2}{\tau_{1}} & {\left[N_{1}^{i f c e} J^{i f c e}\left(\epsilon \mid m_{e} c^{2}, \Gamma_{i f c e}\right)\right.} \\
& +N_{1}^{2 g} J^{2 g}\left(\epsilon \mid m_{e} c^{2}, \Gamma_{2 g}\right) \\
& +N_{1}^{\text {Hce }} H\left(\epsilon \mid m_{e} c^{2}, \Gamma_{\text {Hce }}\right) \\
& +N_{1}^{\text {Hece }} H\left(\epsilon \mid m_{e} c^{2}, \Gamma_{\text {Hece }}\right) \\
& \left.+N_{1}^{r c} G\left(\epsilon \mid m_{e} c^{2}, \Gamma_{r c}\right)\right] .
\end{aligned}
$$

The composite $3 \gamma$ annihilation continuum is composed of contributions from each of the four Ps-producing processes. The total continuum is given by

$$
\begin{array}{r}
\frac{d Q_{3 \gamma}}{d \epsilon}(\epsilon)=\frac{3}{\tau_{3}} \int d \epsilon^{\prime}\left[\frac{1}{N_{3 \gamma}} \frac{d N_{3 \gamma}}{d \epsilon^{\prime}}\left(\epsilon^{\prime}\right)\right]\left\{N_{3}^{i f c e} J^{i f c e}\left(\epsilon \mid \epsilon^{\prime}, \Gamma_{i f c e}\right)+N_{3}^{2 g} J^{2 g}\left(\epsilon \mid \epsilon^{\prime}, \Gamma_{2 g}\right)\right. \\
\left.+N_{3}^{H c e} G\left(\epsilon \mid \epsilon^{\prime}, \Gamma_{H c e}\right)+N_{3}^{H e c e} G\left(\epsilon \mid \epsilon^{\prime}, \Gamma_{H e c e}\right)+N_{3}^{r c} G\left(\epsilon \mid \epsilon^{\prime}, \Gamma_{r c}\right)\right\}
\end{array}
$$

where we have convolved the distribution of equation (21) with the line shapes for each mode to approximate the effect of Doppler broadening.

\section{Applications}

We will apply the results of the previous sections to positron annihilation in four different environments. We will consider a completely ionized medium ( $\S 7.1)$, a completely neutral medium ( $\S 7.2)$, and the intermediate case of a partially-ionized medium ( $\$ 7.3)$. These cases 
will provide insight on how the line shape and ${ }^{3} \mathrm{Ps}$ continuum depend on the physical conditions of the medium. This insight will help understand the results when we then consider the fourth environment: a specific model solar atmosphere (model C of Vernazza et al. (1981); $\S 7.4)$ which provides a fixed relation between temperature, density and ionization fractions.

The important diagnostic features of annihilation radiation are the $Q_{3 \gamma} / Q_{2 \gamma}$ ratio and the shape of the line. We will characterize the shape of the line with its full-width at half maximum (FWHM). However, because the line is a composite of the many modes of annihilation, its shape can be far from Gaussian, and we caution that such a simple characterization may not fully represent its complexity. In particular, when the broad inflight component is present, we will also use the the ratio of the broad component yield to the sum of the yields of the narrow components, $\mathrm{R}_{\mathrm{b} / \mathrm{n}}$, as an additional measure of the line shape. In the following we show how the line shape and $Q_{3 \gamma} / Q_{2 \gamma}$ depend upon the conditions of the medium where the annihilation occurs. The medium is characterized by the temperature $T$, the total (neutral + ionized) $\mathrm{H}$ density $n_{\mathrm{H}}$, and the ionization fractions $X_{\mathrm{H}^{+}}, X_{\mathrm{He}^{+}}$and $X_{\mathrm{He}^{++}}$. The He abundance is fixed at $n_{\mathrm{He}} / n_{\mathrm{H}}=0.1$ (Anders \& Grevesse 1989).

\subsection{Fully Ionized Medium}

We set $X_{\mathrm{H}^{+}}=X_{\mathrm{He}^{++}}=1, X_{\mathrm{He}^{+}}=0$ and calculate the line shape and $Q_{3 \gamma} / Q_{2 \gamma}$ for several independent choices of $T$ and $n_{\mathrm{H}}$. In such a medium, annihilation can occur only by direct annihilation with free electrons and Ps formation through radiative combination with free electrons. Both of these processes produce a $2 \gamma$ line with a Gaussian shape whose width depends only on temperature and is given by equation (15) (see §5.1).

We show $Q_{3 \gamma} / Q_{2 \gamma}$ as a function of $T$ for several densities in Figure 10. The decrease of the ratio up to a few times $10^{5} \mathrm{~K}$ is due to the increasing rate of positronium quenching with temperature by electrons and $\mathrm{H}^{+}$(see panel (a) of Figure 7). At higher temperatures, the decrease is because $2 \gamma$ annihilation with free electrons dominates over Ps formation via radiative combination (see Figure 6).

In Figure 11, $Q_{3 \gamma} / Q_{2 \gamma}$ is plotted against $n_{\mathrm{H}}$ for several temperatures. For each temperature, the ratio is constant for densities $\lesssim 10^{13}$. This is because at these densities quenching is not important and both Ps formation through radiative combination with free electrons and direct annihilation with free electrons are proportional to the electron density.

The profile of the $2 \gamma$ line and $3 \gamma$ continuum for $T=10^{4}$ and $10^{6} \mathrm{~K}$ with $n_{\mathrm{H}}=10^{13}$ $\mathrm{cm}^{-3}$ are shown in panels (a) and (b) of Figure 12, respectively. The line is Gaussian with width given by equation (15) and the contribution from the two components are shown. 
At lower temperature, radiative combination dominates while at higher temperature, direct annihilation dominates.

In Figure 13 we plot the $Q_{3 \gamma} / Q_{2 \gamma}$ ratio versus the FWHM line width for several densities. Because both of the processes responsible for the line produce Gaussian line shapes with widths depending only on temperature and given by equation (15), the widths correspond directly to temperatures and the corresponding temperature scale is shown at the top of the figure.

\subsection{Completely Neutral Medium}

In a completely neutral medium $\left(X_{\mathrm{H}^{+}}=X_{\mathrm{He}^{+}}=X_{\mathrm{He}^{++}}=0\right)$, annihilation only occurs via Ps (formed by in-flight and thermal charge exchange) and via direct annihilation with bound electrons. At the low temperatures consistent with the assumption of a neutral medium, the contribution from thermal charge exchange is small because of its high $6.8 \mathrm{eV}$ threshold (the high-energy tail of the positron Maxwellian is too weak). The $Q_{3 \gamma} / Q_{2 \gamma}$ ratio for $T=4000,5000$ and $6000 \mathrm{~K}$ is shown as a function of density by the dashed curves in Figure 11. At densities greater than $\sim 10^{13} \mathrm{~cm}^{-3}$, the ratio falls with increasing density due to quenching of ${ }^{3} \mathrm{Ps}$ by $\mathrm{H}$. At densities $\lesssim 10^{13} \mathrm{~cm}^{-3}$, quenching is not important and the ratio is constant. At $4000 \mathrm{~K}$, for example, $Q_{3 \gamma} / Q_{2 \gamma}=3.7$. Because the contribution from direct annihilation with bound electrons at such a low temperature is negligible, this value can be directly derived. From Figure 4, the fraction of positrons forming positronium in flight is $f_{1}=0.95$ for $X_{\mathrm{H}^{+}}=0$ so that the fraction of positrons annihilating directly with bound electrons is $1-f_{1}$. Therefore, $Q_{3 \gamma} / Q_{2 \gamma}=2.25 f_{1} /\left(0.5 f_{1}+2\left(1-f_{1}\right)\right)=3.7$ for $f_{1}=0.95$.

The composite line profile (along with the $2 \gamma$ line components and the $3 \gamma^{3}$ Ps continuum) is shown in panel (a) of Figure 14 for $T=5000 \mathrm{~K}$ and a low density of $n_{\mathrm{H}}=10^{9} \mathrm{~cm}^{-3}$ where quenching is not important. At this temperature, the $2 \gamma$ annihilation line is composed mostly of contributions from direct annihilation with bound electrons and a strong contribution from charge exchange in flight.

At higher densities, ${ }^{3}$ Ps quenching modifies the line shape and intensity. Quenching of ${ }^{3}$ Ps produced by thermalized positrons effectively removes three photons from the $3 \gamma$ continuum and adds two photons to the narrow $2 \gamma$ lines $(<2 \mathrm{keV}$ FWHM at the temperatures consistent with a neutral assumption). Quenching of primary in-flight ${ }^{3} \mathrm{Ps}$ via spin-flip adds two photons to the broad $(\sim 6.1 \mathrm{keV})$ in-flight $2 \gamma$ line. Quenching of primary in-flight ${ }^{3} \mathrm{Ps}$ via ionization produces second-generation positrons which either thermalize, contributing to the narrow $2 \gamma$ line components, or form Ps again in flight. This second-generation in- 
flight Ps either annihilates (to produce the intermediate-width $\sim 2.6 \mathrm{keV}$ line (see $§ 3.2$ ) and $3 \gamma$ continuum) or is again quenched. Because most of the Ps atoms formed from primary positrons have energies greater than $10 \mathrm{eV}$ (see Figure 5), quenching via ionization is favored over spin-flip due to their respective cross sections (see Figures 3 and panel (d) of Figure 7). Therefore, the overall effect of quenching of primary in-flight Ps is to increase the yield of the narrower lines relative to the broad in-flight line. Panel (b) of Figure 14 shows the calculated line profile for $T=5000 \mathrm{~K}$ and $n_{\mathrm{H}}=10^{17} \mathrm{~cm}^{-3}$. Comparing with panel (a) for $10^{9} \mathrm{~cm}^{-3}$, the $3 \gamma$ continuum is now absent, the narrow components from thermal positrons annihilating via charge-exchange Ps and directly with bound electrons are much stronger, the intensity of the broad in-flight component is somewhat increased (due to spin-flip quenching), and there is now a contribution from second-generation in-flight charge exchange.

For the high-density case of panel (b), the line is dominated by the narrow components and the formal FWHM is $\sim 1.7 \mathrm{keV}$ (see $§ 5.1$ ), reflecting only these narrow components. For the low-density case of panel (a), the broad in-flight component is much stronger, yet the formal FWHM is still only $\sim 2.6 \mathrm{keV}$, intermediate between the widths of the narrow lines and the broad line and reflecting neither. These examples clearly show that, except for the fully-ionized case where the broad in-flight component is never present, characterizing the shape of the annihilation line with a simple measure of its "width" such as FWHM may not reveal the important processes or the true shape of the line. Even when the broad in-flight component is relatively weak, as in the high-density neutral case of panel (b), its presence can be important when fitting high spectral resolution spectral data with sufficiently good statistical significance. When the in-flight component is present, the line is essentially composed a broad line and a narrow line. In this case, a better representation of the shape of the line is $R_{b / n}$ : the ratio of the broad component yield to the sum of the yields of the narrow components. The curves in Figure 15 show $Q_{3 \gamma} / Q_{2 \gamma}$ versus $\mathrm{R}_{\mathrm{b} / \mathrm{n}}$ for $T=4000,5000$ and $6000 \mathrm{~K}$ and for various densities as noted along each temperature curve. The trend of decreasing $Q_{3 \gamma} / Q_{2 \gamma}$ and $\mathrm{R}_{\mathrm{b} / \mathrm{n}}$ with increasing density along each curve is due to increased quenching: $3 \gamma$ photons are moved into the $2 \gamma$ line which decreases $Q_{3 \gamma} / Q_{2 \gamma}$ and the resulting build-up of the narrow $2 \gamma$ line relative to the broad in-flight line decreases $R_{b / n}$. The trend of decreasing $Q_{3 \gamma} / Q_{2 \gamma}$ and $\mathrm{R}_{\mathrm{b} / \mathrm{n}}$ with decreasing temperature for a given density is due to the shift from Ps formation via thermal charge exchange to direct annihilation with bound electrons as temperature decreases. 


\subsection{Partially-Ionized Medium}

Increasing the ionization fraction of the medium decreases Ps formation via charge exchange (by both in-flight and thermalized positrons) because of the decreased availability of neutral atoms. In-flight Ps formation is further reduced because the larger positron energy loss resulting from increased electron density causes more positrons to thermalize rather than undergo charge exchange in flight. This is reflected in a decrease of both $f_{1}$ (the fraction of positrons forming Ps in flight) and $Q_{3 \gamma} / Q_{2 \gamma}$. The effect on the line profile is a reduction of the broad in-flight component and a corresponding increase of the narrow component resulting in a smaller $\mathrm{R}_{\mathrm{b} / \mathrm{n}}$. Line profiles are shown in Figure 16 for several values of $X_{\mathrm{H}^{+}}$ $(0.0,0.01,0.10$ and 0.50$)$ for $T=5000 \mathrm{~K}$ and $n_{\mathrm{H}}=10^{15} \mathrm{~cm}^{-3}$. The inset table gives the associated values of $f_{1}, Q_{3 \gamma} / Q_{2 \gamma}$, formal line width and $\mathrm{R}_{\mathrm{b} / \mathrm{n}}$.

We have compared our results with the recent positron annihilation calculations of Churazov et al. (2005) for a low-density, pure $\mathrm{H}$ medium with $T=8000 \mathrm{~K}$ and $X_{\mathrm{H}^{+}}=0.1$. For the line shapes we find good agreement: a width of the broad line from in-flight charge exchange of $\sim 6 \mathrm{keV}$ (compared with $\sim 5.9 \mathrm{keV}$ here) and a width of the narrow line from thermal charge exchange of $\sim 1.4 \mathrm{keV}$ (compared with $\sim 1.3 \mathrm{keV}$ here). However, we disagree on $f_{1}$, the fraction of positrons that form Ps in flight. While Churazov et al. (2005) do not provide values for $f_{1}$, they show in their Figure 11 the fraction of the total line radiation contributed by the broad in-flight line which is directly related. Our values for this fraction are consistently higher than theirs; for example, at $X_{\mathrm{H}^{+}}=0.1$, they found $\sim 0.31$ for this fraction compared with $\sim 0.52$ from our calculations. This is similar to the difference between our calculations for $f_{1}$ and those of BRD (see $\S 3.1$ ).

\subsection{Solar Atmosphere Model}

We now calculate the annihilation line shape and $Q_{3 \gamma} / Q_{2 \gamma}$ throughout the fourth environment: the quiet solar atmosphere represented by the non-LTE calculations of Vernazza et al. (1981) (model C). The density, temperature and ionization fraction vary over wide ranges in such a model and it will be instructive to see how the annihilation radiation varies in response. We caution that atmospheric conditions during a solar flare may be quite different than those of such a model. Indeed, as we will see in $\S 8$, observations of the annihilation line from solar flares are revealing an atmosphere that can be surprisingly different. We also caution that, in an atmosphere such as Vernazza et al. (1981), temperatures and ionization fractions can change rapidly over very narrow ranges of height (and corresponding grammage). As a result, at any given point there may not be sufficient material for all of the positrons to remain near that point as they slow down and annihilate. Nevertheless, the 
calculation will demonstrate the range of $Q_{3 \gamma} / Q_{2 \gamma}$ and line shapes possible in such a model atmosphere. An accurate calculation of positron annihilation must consider the location of positron emitter production and transport of the positrons, with the result that the line may be composed of contributions from a range of conditions in the solar atmosphere.

In Figure 17 we show $n_{\mathrm{H}}$ and $n_{e}$ as a function of temperature for the Vernazza model using the tabulated values of $T, n_{\mathrm{H}}$ and $n_{e}$ (Vernazza et al. 1981). (The numbers along the $\mathrm{H}$ curve indicate regions that will be referred to in the following discussions.) Vernazza et al. (1981) do not specify the ionization fractions for $\mathrm{H}$ and $\mathrm{He}$ (i.e., $X_{\mathrm{H}^{+}}, X_{\mathrm{He}^{+}}$and $X_{\mathrm{He}^{++}}$). We estimate these parameters in the following manner. Up to the temperature where $n_{\mathrm{e}}=n_{\mathrm{H}}$ $\left(T=\simeq 2.1 \times 10^{4} \mathrm{~K}\right)$, we assume that He is totally neutral; i.e., the free electrons are due solely to ionized $\mathrm{H}$. Above this temperature we assume that $\mathrm{H}$ is totally ionized and the excess $n_{\mathrm{e}}$ is due solely to singly-ionized $\mathrm{He}\left(\mathrm{He}^{+}\right)$. Above $T \gtrsim 3.7 \times 10^{4} \mathrm{~K}$ (at which $n_{\mathrm{e}} / n_{\mathrm{H}}=1.1$ ), we assume that there is no neutral $\mathrm{He}(\mathrm{He} / \mathrm{H}$ is assumed to be $10 \%)$. We thus have three distinct temperature regimes where the ionization fractions have the values $\left(X_{\mathrm{H}^{+}}, 0,0\right),\left(1, X_{\mathrm{He}^{+}}, 0\right)$ and $\left(1,1-X_{\mathrm{He}^{++}}, X_{\mathrm{He}^{++}}\right)$. For a given temperature, then, there is only one free ionization parameter which is set by the ratio $n_{\mathrm{e}} / n_{\mathrm{H}}$ tabulated by Vernazza et al. (1981). All of these parameters can be related to the height in the atmosphere using Table 12 of Vernazza et al. (1981).

In Figure 18, $Q_{3 \gamma} / Q_{2 \gamma}$ is plotted as a function of temperature (with density increasing along the curve as shown by the arrow). At the region labeled (1), the conditions are similar to the completely neutral case discussed above, with density decreasing rapidly from $\sim 10^{15}$ to $\sim 10^{13} \mathrm{~cm}^{-3}$. As quenching decreases, the ratio increases until it reaches the maximum of $\sim 4$ (see the neutral curve of Figure 11 for $T=6000 \mathrm{~K}$ ). At region (2), $\mathrm{H}$ is partially ionized and He is neutral. At these temperatures and ionization states, annihilation is dominated by Ps formation (via radiative combination and both thermal and in-flight charge exchange) and the ratio reaches its maximum possible value of 4.5 (i.e., $(0.75 \times 3) /(0.25 \times 2))$ since the density is too low for quenching to be important. Along region (3) to (4), $Q_{3 \gamma} / Q_{2 \gamma}$ drops rapidly due to $\mathrm{H}$ becoming fully ionized and the ratio reaches its value of $\lesssim 3$ for fully ionized as shown in Figure 10 for $n_{\mathrm{H}} \simeq 10^{10} \mathrm{~cm}^{-3}$ and $T \simeq 2 \times 10^{4} \mathrm{~K}$. In region (6), there are no neutral atoms and any $\mathrm{Ps}$ formation is due to radiative combination with free electrons. $Q_{3 \gamma} / Q_{2 \gamma}$ continues to decrease due to increased direct annihilation with free electrons as temperature increases (see Figure 6). The same labeling of the different regions is employed in Figure 19 where $Q_{3 \gamma} / Q_{2 \gamma}$ is plotted versus density. We see that for such an atmosphere, a given value of $Q_{3 \gamma} / Q_{2 \gamma}$ can occur at several values of density.

In Figure 20 we show calculated line shapes at several locations in the atmosphere. In panel (a), $\mathrm{T}=5000 \mathrm{~K}$ and $n_{\mathrm{H}} \simeq 3 z \times 10^{14} \mathrm{~cm}^{-3}$, corresponding to region (1) of Figure 
17. The narrow component is due primarily to direct annihilation with bound electrons and is strong relative to the broad component due to Ps formed in flight. ${ }^{3}$ Ps quenching results in the contribution from second-generation in-flight Ps. In panel (b) of Figure 20, $\mathrm{T}=6000 \mathrm{~K}$ and $n_{\mathrm{H}} \simeq 2 \times 10^{13} \mathrm{~cm}^{-3}$ and the broad component from Ps formed in flight reaches its relative maximum (the narrow peak between regions (1) and (2) of Figure 18). The narrow component now has a significant contribution from thermal charge exchange due to the increasing temperature and quenching is no longer significant. In panel (c) of Figure 20, $\mathrm{T}=7000 \mathrm{~K}$, corresponding to $n_{\mathrm{H}} \simeq 10^{11} \mathrm{~cm}^{-3}$ and region (2) of Figure 18, and the broad in-flight charge exchange component is reduced due to increased ionization. The narrow component is due to thermal charge exchange and, because of the increased ionization, radiative combination and direct annihilation with free electrons. In panel (d) of Figure 20, $\mathrm{T}=10^{4} \mathrm{~K}$, corresponding to $n_{\mathrm{H}} \simeq 5 \times 10^{10} \mathrm{~cm}^{-3}$ (region (3) of Figure 18). The broad component is negligible and the narrow component is due primarily to thermal charge exchange with the remaining neutral atoms. At higher temperatures (lower densities), thermal charge exchange is negligible due to the high degree of ionization, and the line is formed by direct annihilation and radiative combination with free electrons. The lines are Gaussian with the FWHM given by equation (15). The calculated line shapes at $10^{5}$ and $10^{6} \mathrm{~K}$ are shown panels (e \& f) of Figure 20.

In Figures 21 and 22 we plot the formal FWHM versus density and temperature, respectively and in Figure 23 we plot the $Q_{3 \gamma} / Q_{2 \gamma}$ ratio versus formal FWHM. In all three of these figures we also plot the broad/narrow ratio, $\mathrm{R}_{\mathrm{b} / \mathrm{n}}$. When $\mathrm{R}_{\mathrm{b} / \mathrm{n}}$ is greater than $\sim 0.05$, the formal FWHM no longer reliably reflects the shape of the line and we emphasize this by making the FWHM curve dotted in this region. Detailed measurements of the line with high-resolution detectors such as RHESSI can reveal the true shape of the line and alter conclusions based on FWHM alone. We note that only in the high-temperature/low-density regions (regions $3-6$ of Figure 21) does the formal FWHM ever exceed the value of $\sim 1.7 \mathrm{keV}$. We also see that the relationships are not unique, so that measurement of just one quantity does not determine where in the atmosphere the annihilations are taking place.

\section{Application to RHESSI Data}

RHESSI (Lin et al. 2002) made the first high-resolution spectral observation of the solar positron annihilation line during the 2002 July 23 solar flare (Share et al. 2003). The line was subsequently observed in both the 2003 October 28 and November 2 flares (Share et al. 2004). When Share et al. (2004) fit the annihilation line with a Gaussian, the measured width during all three flares was surprisingly broad, with a FWHM of $4-8 \mathrm{keV}$. The October 
28 flare was remarkable in that during the first 10 minutes of the observation the line was broad at an approximately constant value, narrowed dramatically over an $\sim 2$-minute interval to $\sim 1 \mathrm{keV}$, and then remained approximately constant at this value during the remainder of the observation ( 12 minutes). Except for the first four minutes of the October 28 flare, the measured $Q_{3 \gamma} / Q_{2 \gamma}$ ratio in all of the flares was consistent with zero.

In the previous analyses, the data were compared with calculations obtained from the same computer codes used in the current paper, but the cross sections for the various interactions were not yet updated and the process of in-flight charge exchange of second-generation positrons was not included. Since those analyses, RHESSI has observed the annihilation line in at least one other flare, that of 2005 January 20. The analyses of the data from all of these flares continue as improvements in the analysis techniques become available. In this section we compare the calculations with data but only as an example of what can be done with positron annihilation line measurements rather than trying to provide definitive answers. We will consider RHESSI observations of only the October 28 and November 2 flares because both of these flares were more intense than the July 23 flare, providing data with significantly-improved statistical quality.

We begin with the November 2 flare. In their analysis of this flare, Share et al. (2004) obtained an acceptable fit to the annihilation line spectrum using a $4.8 \pm 0.5 \mathrm{keV}$ (FWHM) Gaussian. Using the new calculation, we find that a Vernazza quiet-Sun atmosphere at $T \simeq 5000 \mathrm{~K}$ also provides an acceptable fit ( $13 \%$ probability based on $\left.\chi^{2}\right)$ to the $500-520$ $\mathrm{keV}$ spectrum shown in panel (a) of Figure 24; the different components of the model are plotted in panel (a) of Figure 20. However, we do not believe we can confidently conclude that the annihilation occurred in this environment. The $0.01 \mathrm{gm} \mathrm{cm}^{-2}$ overlying column depth and $\sim 3 \times 10^{14} \mathrm{~cm}^{-3}$ density at this height in the quiet-Sun atmosphere are only marginally sufficient for production of the radioactive positron emitters by accelerated particles with a typical power law index of $\sim 4$. The spectrum for this flare may be considerably harder (Share et al. 2004), implying a large fraction of positrons from the decay of pions whose highenergy production reactions require an even greater column depth. Under these conditions a significant fraction of the annihilation line could be Compton scattered, with the scattered continuum contributing to the apparent $Q_{3 \gamma} / Q_{2 \gamma}$ ratio. The measured $99 \%$ upper limit on the ratio (1.3) is only marginally consistent with the calculated ratio (1.1) even without a contribution from Compton scattering.

We plot the line spectrum accumulated during the first 2-minutes of the October 28 flare in panel (b) of Figure 24. It is clearly too broad to be consistent with the calculated shape produced by annihilation in a quiet-Sun atmosphere at $5000 \mathrm{~K}$. The only way to obtain a broader line at temperatures $<10^{4} \mathrm{~K}$ is to increase the relative contribution from in-flight 
charge exchange. The maximum contribution in the Vernazza atmosphere occurs at a temperature of $6000 \mathrm{~K}\left(\mathrm{R}_{\mathrm{b} / \mathrm{n}} \simeq 3.9\right.$, see Figure 22 and panel (b) of Figure 20). The calculated shape for this environment (solid curve) provides a marginally-acceptable fit $(\sim 1.2 \%$ confidence). Other considerations exclude this origin, however: the $6 \times 10^{-4} \mathrm{gm} \mathrm{cm}^{-2}$ overlying column depth and $\sim 2 \times 10^{13} \mathrm{~cm}^{-3}$ density are much too small for sufficient nuclear reactions and the calculated $Q_{3 \gamma} / Q_{2 \gamma}$ ratio (3.5) is inconsistent with the measured ratio (1.8 \pm 0.4$)$. We also fit the data with line shapes derived assuming the F1 flare atmosphere of Machado et al. (1980) but no acceptable fit to the line could be obtained since $R_{\mathrm{b} / \mathrm{n}}$ never exceeds 1.5 in the cool regions of such an atmosphere. The alternative scenario is annihilation in a high-temperature region where the line is a thermally-broadened Gaussian. Panel (b) of Figure 24 shows this Gaussian fit (dotted curve; $6.7 \pm 1.5 \mathrm{keV}$ FWHM). From equation (15), this width corresponds to $T=3-4 \times 10^{5} \mathrm{~K}$. At this temperature, $\mathrm{H}$ and He would be ionized and Figures 10, 11 and 13 can be used. Figure 13 shows that the densities required for adequate positron production $\left(\gtrsim 10^{15} \mathrm{~cm}^{-3}\right)$ correspond to $Q_{3 \gamma} / Q_{2 \gamma}$ ratios $\lesssim 0.2$ which are significantly less than the measured value $(1.8 \pm 0.4)$. However, such small values of $Q_{3 \gamma} / Q_{2 \gamma}$ may be allowed since much of the continuum below the $511 \mathrm{keV}$ line may be due to Compton scattering of the line at the large depths where pion-decay positrons annihilate. We base this argument on the hardness of the accelerated particle spectrum and detection of the characteristic pion-decay $\gamma$-ray spectrum observed by CORONAS-F (Kuznetsov et al. 2005). We therefore confirm the Share et al. (2004) conclusion that positron annihilation during the early part of the October 28 flare occurred in a high temperature region $\left(T=3-4 \times 10^{5} \mathrm{~K}\right)$ at densities $\gtrsim 10^{15} \mathrm{~cm}^{-3}$.

In panel (c) of Figure 24 we show that an $\sim 1$ keV FWHM Gaussian (dotted curve) provides an excellent fit to the narrow line observed during the last 10 minutes of the October 28 flare (Share et al. 2004) when the measured $99 \%$ upper limit for the $Q_{3 \gamma} / Q_{2 \gamma}$ ratio is 0.3. Such a narrow width requires both that the temperature be less than $10^{4} \mathrm{~K}$ and that the broad/narrow ratio $R_{b / n}$ be sufficiently small. We find that there is no temperature in either the Vernazza atmosphere or a neutral medium at which this can be achieved and also satisfy the $Q_{3 \gamma} / Q_{2 \gamma}$ constraint (see Figures 15 and $18 \& 22$ ). An acceptable fit can be achieved, however, by increasing the ionization fraction which reduces $\mathrm{R}_{\mathrm{b} / \mathrm{n}}$ (see Figure 16). Assuming $T=5000 \mathrm{~K}$ and a density of $10^{15} \mathrm{~cm}^{-3}$ (which satisfies both the $Q_{3 \gamma} / Q_{2 \gamma}$ constraint and the nuclear production requirement), we find that an ionization fraction of at least $20 \%$ is required to provide an acceptable fit $(>1 \%$ confidence) to the spectrum. The solid curve in panel (c) of Figure 24 shows this fit.

In summary, we find that positron annihilation in solar flares occurs in a wide range of conditions. The line shape observed during the November 2 flare was adequately fit with the quiet solar atmosphere of Vernazza et al. (1981) at $T=5000 \mathrm{~K}$. When the line was 
broad during the October 28 flare, the data suggest that the environment was ionized with

$T=3-4 \times 10^{5} \mathrm{~K}$ at a density $\gtrsim 10^{15} \mathrm{~cm}^{-3}$. When the line was narrow, the environment was relatively cool at $\sim 5000 \mathrm{~K}$ yet the $\mathrm{H}$ ionization fraction had to have been at least $20 \%$. We emphasize again that these results are meant only as an example of the application of the calculations to data. More detailed analyses of these flares will be presented in a subsequent paper.

\section{Summary}

The formation of the positron annihilation line is a complex interplay of competing processes. In this paper we explored this process, identifying the various annihilation modes, determining the nature of the contribution of each mode, and establishing how the physical conditions of the environment determine their relative contributions. We thoroughly updated the cross sections for all of the relevant processes with recent laboratory measurements and theoretical calculations, providing reliable cross sections for all of the important interactions. We calculated the relative rates for each of the modes, their associated line shapes, and how the individual contributions combine to produce the final composite line shape. We considered neutral, fully ionized, and partially ionized mediums, and the quiet sun atmosphere of Vernazza et al. (1981).

In a fully ionized medium with $T \gtrsim 10^{4} \mathrm{~K}, Q_{3 \gamma} / Q_{2 \gamma}$ is less than $\sim 3$ and decreases with increasing density (due to collisional quenching of ${ }^{3} \mathrm{Ps}$ ) and increasing temperature (due to direct annihilation dominating over Ps formation via radiative combination). The width of the line depends directly on temperature as given by equation (15). In a neutral medium, $Q_{3 \gamma} / Q_{2 \gamma}$ reaches its maximum value at low densities where quenching is not important (for $\left.T=5000 \mathrm{~K}, Q_{3 \gamma} / Q_{2 \gamma} \simeq 3.6\right)$. The line is composed of a narrow component $(\sim 1.5 \mathrm{keV})$ due to direct annihilation with bound electrons and a broad component $(\sim 6.1 \mathrm{keV})$ due to charge exchange in flight with neutral $\mathrm{H}$. The latter can contribute significantly to the line shape when the density is low and quenching is not important. The effect of increasing the ionization fraction is to decrease Ps formation via charge exchange (by both in-flight and thermalized positrons) due to the decreasing availability of neutral atoms which reduces the broad component relative to the narrow components.

For the model C solar atmosphere of Vernazza et al. (1981), we calculated the annihilation line shape and the $Q_{3 \gamma} / Q_{2 \gamma}$ ratio throughout. Such a model provides a wide range of physical conditions and we identified how these conditions affect the line shape and $Q_{3 \gamma} / Q_{2 \gamma}$. With such an understanding, analyses of annihilation line observations from solar flares can provide reliable constraints on the physical conditions where the positrons annihilate. 
We compared the calculated line shapes and $Q_{3 \gamma} / Q_{2 \gamma}$ ratios with high spectral resolution data recently obtained with RHESSI from the 2003 October 28 and November 2 flares. We found that positron annihilation in solar flares can occur in a wide range of conditions. The annihilation environment of the November 2 flare could have been at a temperature of 5000 $\mathrm{K}$ in the quiet solar atmosphere of Vernazza et al. (1981). When the line was broad during the October 28 flare, the data suggest that the environment was ionized with $T=3-4 \times 10^{5}$ $\mathrm{K}$ and density $\gtrsim 10^{14} \mathrm{~cm}^{-3}$. When the line was narrow, the environment was relatively cool at $\sim 5000 \mathrm{~K}$ yet the $\mathrm{H}$ ionization fraction had to have been at least $20 \%$.

We thank H. R. J. Walters for valuable discussions and for providing unpublished cross sections and T. S. Stein for helpful discussions. We acknowledge N. Guessoum and R. J. Drachman who participated at the beginning of this research. We also acknowledge R. Ramaty, with whom this research was started and for whom this subject was of major interest for many years. B. Kozlovsky acknowledges the Israeli Science Foundation for support. Part of this research was supported by NASA DPR S92680F and W19977 and by ONR.

\section{A. Direct Annihilation Line Spectrum}

Here we show that the line resulting from the direct annihilation of nonrelativistic thermal electrons and positrons has a gaussian distribution with width given by

$$
\mathrm{FWHM}=m c^{2} \sqrt{\ln 2\left(\frac{k T}{m c^{2}}\right)}=1.1 \mathrm{keV} \sqrt{\frac{T}{10^{4} \mathrm{~K}}} .
$$

In a free annihilation the emitted photon energies in the lab system are

$$
\epsilon=\left(1+\frac{E_{+}+E_{-}}{m_{e} c^{2}}\right)\left(1 \pm \beta_{c m} \mu^{*}\right) m_{e} c^{2}
$$

where $E_{+}$and $E_{-}$are the kinetic energies of the positron and electron, $u \equiv c \beta_{c m}$ is the magnitude of the velocity of the center of mass, and $\mu^{*}$ is the cosine of the angle between one of the emitted photons and the electron in the CM system. Again, all starred quantities

refer to the $\mathrm{CM}$ system. At nonrelativistic energies $\left(E_{ \pm} \ll m_{e} c^{2}\right)$ this expression reduces to

$$
\epsilon \simeq\left(1 \pm \beta_{c m} \mu^{*}\right) m_{e} c^{2}
$$

The cross section differential in the cosine of the polar angle $\mu^{*}$ is (e.g. Heitler 1954)

$$
\frac{d \sigma^{*}}{d \mu^{*}}=\frac{\pi r_{0}^{2}}{4} \frac{1}{\gamma^{* 2} \beta^{*}}\left\{\frac{\gamma^{* 2}+\left(\gamma^{* 2}-1\right)\left(2-\mu^{* 2}\right)}{\gamma^{* 2}-\left(\gamma^{* 2}-1\right) \mu^{* 2}}-\frac{2\left(\gamma^{* 2}-1\right)^{2}\left(1-\mu^{* 2}\right)^{2}}{\left[\gamma^{* 2}-\left(\gamma^{* 2}-1\right) \mu^{* 2}\right]^{2}}\right\} .
$$


In the nonrelativistic limit the photons are emitted isotropically in the CM system because this cross section reduces to

$$
\frac{d \sigma^{*}}{d \mu^{*}} \simeq \frac{\pi r_{0}^{2} c}{2 v},
$$

which is independent of angle and only depends on the relative velocity $v=\left|\mathbf{v}_{+}-\mathbf{v}_{-}\right|$. This holds even with the inclusion of the Coulomb correction factor $(2 \pi \alpha c / v) /\left(1-e^{-2 \pi \alpha c / v}\right)$ in which case the cross section becomes

$$
\frac{d \sigma^{*}}{d \mu^{*}} \simeq \frac{\pi r_{0}^{2}}{2} \frac{2 \pi \alpha(c / v)^{2}}{1-e^{-2 \pi \alpha c / v}}
$$

The annihilation spectrum is given by

$$
\begin{aligned}
\frac{d N_{\gamma}}{d t d \epsilon d^{3} \mathbf{x}}(\epsilon)=2 n_{+} n_{-}\left(\frac{m_{e}}{2 \pi k T}\right)^{3} \int d^{3} \mathbf{v}_{+} d^{3} \mathbf{v}_{-} & \\
& \quad \exp \left(-\frac{m_{e} v_{+}^{2}}{2 k T}\right) \exp \left(-\frac{m_{e} v_{-}^{2}}{2 k T}\right) v \frac{d \sigma^{*}}{d \mu^{*}}(v)\left|\frac{d \mu^{*}}{d \epsilon}\right| .
\end{aligned}
$$

Equation (A3) gives

$$
\left|\frac{d \mu^{*}}{d \epsilon}\right|=\frac{1}{m_{e} c u} .
$$

Equation (A3) also implies that to obtain a photon of energy $\epsilon$ the minimum CM velocity is

$$
u_{0}(\epsilon)=c\left(\frac{\epsilon}{m_{e} c^{2}}-1\right) .
$$

Under the coordinate transformation $\left\{\mathbf{v}_{+}, \mathbf{v}_{-}\right\} \rightarrow\{\mathbf{u}, \mathbf{v}\}$, where $\mathbf{u}$ is the velocity of the center of mass, the annihilation spectrum becomes

$$
\begin{aligned}
\frac{d N_{\gamma}}{d t d \epsilon d^{3} \mathbf{x}}(\epsilon) & =2 n_{+} n_{-}\left(\frac{m_{e}}{2 \pi k T}\right)^{3} \int d^{3} \mathbf{u} d^{3} \mathbf{v} \exp \left(-\frac{m_{e} u^{2}}{k T}\right) \exp \left(-\frac{m_{e} v^{2}}{4 k T}\right) v \frac{d \sigma^{*}}{d \mu^{*}}(v) \frac{1}{m_{e} c u} \\
& =\frac{2 n_{+} n_{-}(4 \pi)^{2}}{m_{e} c}\left(\frac{m_{e}}{2 \pi k T}\right)^{3}\left\{\int_{0}^{\infty} d v v^{2} \exp \left(-\frac{m_{e} v^{2}}{4 k T}\right) v \frac{d \sigma^{*}}{d \mu^{*}}(v)\right\} \quad(\mathrm{A} 10) \\
\left\{\int_{u_{0}(\epsilon)}^{\infty} d u u \exp \left(-\frac{m_{e} u^{2}}{k T}\right)\right\} . &
\end{aligned}
$$

The only dependence on $\epsilon$ is through $u_{0}(\epsilon)$ in the integration over $u$. The integration over $v$ simply yields an overall constant, hence we can write

$$
\begin{aligned}
\frac{1}{N_{\gamma}} \frac{d N_{\gamma}}{d \epsilon}(\epsilon) & \propto \int_{u_{0}(\epsilon)}^{\infty} d u u \exp \left(-\frac{m_{e} u^{2}}{k T}\right) \\
& \propto \exp \left(-\frac{m_{e} u_{0}^{2}}{k T}\right) \\
& =\exp \left[-\frac{m_{e} c^{2}}{k T}\left(\frac{\epsilon}{m_{e} c^{2}}-1\right)^{2}\right]
\end{aligned}
$$


Finally, from (A11) it is a simple matter to show that the full width at half maximum is given by equation (A1).

\section{REFERENCES}

Allen, C. W. 1976, Astrophysical Quantities (Athlone Press:London)

Anders, E. \& Grevesse, N. 1989, Geochim. Cosmochim. Acta, 53, 193

Beers, R. H. \& Hughs, V. W. 1968, 1st Int. Conf. on Atomic Phys., New York

Bhatia, A. K., Drachman, R. J., \& Temkin, A. 1977, Phys. Rev. A, 16, 1719

Blackwood, J. E., McAlinden, M. T., \& Walters, H. R. J. 2002, Phys. Rev. A., 65, 032517

Brown, B. L., \& Leventhal, M. 1987, ApJ, 319, 637

Bussard, R. W., Ramaty, R., \& Drachman, R. J. 1979, ApJ, 228. 928

Campbell, C. P., McAlinden, M. T., Kernoghan, A. A., \& Walters, H. R. J. 1998, Nucl. Instr. \& Meth. B, 143, 41

Campbell, C. P., McAlinden, M. T., MacDonald, F. G. R. S., \& Walters, H. R. J. 1998, Phys. Rev. Lett., 80, 5097

Chapuis, C. G. L., Wallyn, P., \& Durouchoux, P. 1994, ApJS, 92, 545

Churazov, E., Sunyaev, R., Sasonov, S., Revnivtsev, M., \& Varshalovich, D. 2005, Mon. Not. R. Astron. soc., in press

Charlton, M. \& Humberston, J. W. 2001, Positron Physics, (Cambridge University Press:Cambridge)

Chupp, E. L., Forrest, D. J., Higbie, P. R., Suri, A. N., Tsai, C., \& Dunphy, P. P. 1973, Nature, 241, 333

Crannell, C. J., Joyce, G., Ramaty, R., \& Werntz, C. 1976, ApJ, 210, 582

Fraser, P. A. \& Kraidy, M. 1965, Proc. Phys. Soc. 89, 533

Fromme, D., Kruse, G., Raith, W., \& Sinapius, G. 1986, Phys. Rev. Lett., 57, 3031

Gilmore, S., Blackwood, J. E., \& Walters, H. R. J. 2004, Nucl. Instr. \& Meth. B, 221, 124 
Gould, R. J. 1989, ApJ, 344, 232

Guessoum, N., Ramaty, R., \& Lingenfelter, R. E. 1991, ApJ, 378, 170

Guessoum, N., Skibo, J. G., \& Ramaty, R. 1997, in Proceedings of the 2nd Integral Workshop, St. Malo, ESA SP-382, pg. 113

Guessoum, N., Jean, P., Knodlseder, J., Lonjou, V., von Ballmoos, P., \& Weidenspointner, G. 2005, in Proceedings of the 5th Integral Workshop, Munich, ESA SP-552, pg. 57

Guessoum, N., Jean, P., \& Gillard, W. 2005, A\&A, (in press)

Heitler, W. 1954, The Quantum Theory of Radiation, (New York:Dover)

Hoffman, A., Falker, T., Raith, Weber, M., Becker, D. P., \& Lin, K. G. 1997, J. Phys. B: At. Mol. Opt. Phys., 30, 3297

Hua, X.-M., Ramaty, R., \& Lingenfelter, R. E. 1989, ApJ, 341, 516

Huba, J. D. 1994, NRL/PU/6790-94-265

Humberston, J. W. 1974, J. Phys. B., 7, L286

Humberston, J. W. \& Wallace, J. G. G. 1972, J. Phys. B., 5, 1138

Humberston, J. W., Charlton, M., Jacobsen, F. M., \& Deutch. B. I. 1987. J. Phys. B., 20, L25

Jacobsen, F. M., Frandsen, N. P., Knudsen, H., Mikkelsen, U., \& Schrader, D. M. 1995, J. Phys. B: At. Mol. Opt. Phys., 28, 4691

Jean, P., et al. 1987, A\&A, 407, L55

Jones, G. O., Charlton, M., Slevin, J., Larrichia, G., Kover, A., Poulsen, M. R., Nic Chormaic, S. 1993, J. Phys. B: At. Mol. Opt. Phys., 26, L483

Kernoghan, A. A., Robinson, D. J. R., McAlinden, M. T., \& Walters, H. R. J. 1996, J. Phys. B: At. Mol. Opt. Phys., 29, 2089

Kozlovsky, B., Lingenfelter, R. E., \& Ramaty, R. 1987, ApJ, 316, 801

Kozlovsky, B., Murphy, R. J., \& Share, G. H. 2004, ApJ, 604, 892

Kuznetsov, S. N., Kurt, V. G., Yushkov, B. Y., Myagkova, I. N., and Kudela, K. 2005, Submitted to Solar Physics 
Lin, R. P., et al. 2002, Sol. Phys., 210, 3

Lingenfelter, R. E., \& Ramaty, R. 1989, ApJ, 343, 686

Machado, M. E., Avrett, E. H., Vernazza, J. E., \& Noyes, R. W.. 1980, ApJ, 242, 336

Massey, H. S. \& Mohr, C. B. O. 1954, Proc. Phys. Soc. A, 67, 695

Mori, S. \& Sueoka, O. 1994, J. Phys. B: At. Mol. Opt. Phys., 27, 4349

Murphy, R. \& Ramaty, R. 1985, Adv. Space Res., 4, 127

Murphy, R. J., Dermer, C. D., \& Ramaty, R. 1987, ApJS, 63, 721

Omidvar, K. 1965, Phys. Rev., 140, 26

Opal, C. B., Peterson, W. K., \& Beaty, E. C. 1971, J. Chem. Phys., 55, 4100

Overton, N., Mills, R. J., \& Coleman, P. G. 1993, J. Phys. B: At. Mol. Opt. Phys., 26, 3951

Pond, T. A., \& Dicke, R. H. 1952, Phys. Rev., 85, 489

Ramaty, R., Kozlovsky, B., \& Lingenfelter, R. E. 1975, Space Science Rev., 18, 341

Ray, H. 2002, Phys. Let. A, 299, 65

Share, G. H., Chupp, E. L., Forrest, D. J., \& Rieger, E. 1983, Positron-Electron Pairs in Astrophysics, ed. M. L. Burns, A. K. Harding \& R. Ramaty (AIP; New York), 15

Share, G. H., Murphy, R. J., \& Skibo, J. G. 1996, in High Energy Solar Physics Workshop, ed. R. Ramaty, N. Mandzhavidze, (AIP:New York), pg. 162

Share, G. H., Murphy, R. J., Skibo, J. G., Smith, D. M., Hudson, H. S., Lin, R. P., Shih, A. Y., Dennis, B. R., Schwartz, \& R. A., Kozlovsky, B. 2003, ApJ, 595, L85

Share, G. H., Murphy, R. J., Smith, D. M., Schwartz, R. A., \& Lin, R. P. 2004, ApJ, 615, L169

Spicher, G., Olsson, B., Raith, W., Sinapius, G., \& Sperber, W. 1990, Phys. Rev. Lett., 64, 1019

Sperber, W., Becker, D., Lynn, K. G., Raith, W., Schwab, A., Sinapius, G., Spicher, G., \& Webber, M. 1992, Phys. Rev. Lett., 68, 3690

Stecker, F. W. 1971, Cosmic Gamma Rays, NASA SP-249 
Stein, T. S., Harte, M., Jiang, J., Kauppila, W. E., Kwan, C. K., Li, H., \& Zhou, S. 1998, Nucl. Instr. \& Meth. B, 143, 68

Van Reeth, P., Humberston, J. W., Iwata, K., Greaves, R. G., \& Surko, C. M. 1996, J. Phys. B., 29, L465

Vernazza, J. E., Avrett, E. H., \& Loeser, R. 1981, ApJ, 45, 635

Veselovsky, I. S., et al. 2004, Cos. Res., 42, 435

Vestrand, W. T., Share, G. H., Murphy, R. J., Forrest, D. J., Rieger, E., Chupp, E. L., \& Kanbach, G. 1999, ApJS, 120, 409

Wallyn, P., Durouchoux, Ph., Chapuis, C., \& Leventhal, M. 1994, ApJ, 422, 610

Weber, M., Hoffman, A., Raith, W., Sperber, W., Jacobsen, F., \& Lynn, K. G. 1994, Hyperfine Interact., 89, 221

Wu, H., Bray, I., Fursa, D. V., \& Stelbovics, A. T. 2004, J. Phys. B: At. Mol. Opt. Phys., 37,1165

Zhou, S., Li, H., Kauppila, W. E., Kwan, C. K., \& Stein, T. S. 1997, Phys. Rev. A, 55, 361

Zurek, W. H. 1985, ApJ, 289, 603 
Table 1. Interactions Involving Positrons and Positronium

\begin{tabular}{llll}
\hline \hline Energy Redistribution & Positronium Formation & Positronium Quenching & Annihilation \\
\hline$e^{+} \mathrm{H} \rightarrow e^{+} \mathrm{H}^{*}$ & $e^{+} e^{-} \rightarrow \gamma \mathrm{Ps}$ & $e^{-3} \mathrm{Ps} \rightarrow e^{-} e^{-} e^{+}$ & $e^{+} e^{-} \rightarrow 2 \gamma$ \\
$e^{+} \mathrm{H} \rightarrow e^{+} e^{-} \mathrm{H}^{+}$ & $e^{+} \mathrm{H} \rightarrow \mathrm{H}^{+} \mathrm{Ps}$ & $\mathrm{H}^{3} \mathrm{Ps} \rightarrow \mathrm{H} e^{-} e^{+}$ & $e^{+} \mathrm{H} \rightarrow \mathrm{H}^{+} 2 \gamma$ \\
$e^{+} \mathrm{He} \rightarrow e^{+} \mathrm{He}^{*}$ & $e^{+} \mathrm{He} \rightarrow \mathrm{He}^{+} \mathrm{Ps}$ & $e^{-3} \mathrm{Ps} \rightarrow e^{-1} \mathrm{Ps}$ & $e^{+} \mathrm{He} \rightarrow \mathrm{He}^{+} 2 \gamma$ \\
$e^{+} \mathrm{He} \rightarrow e^{+} e^{-} \mathrm{He}^{+}$ & & $\mathrm{H}^{3} \mathrm{Ps} \rightarrow \mathrm{H}^{1} \mathrm{Ps}$ & ${ }^{1} \mathrm{Ps} \rightarrow 2 \gamma$ \\
& & $\mathrm{H}^{+} \mathrm{Ps} \rightarrow \mathrm{H} e^{+}$ & ${ }^{3} \mathrm{Ps} \rightarrow 3 \gamma$ \\
& & $\mathrm{He}^{+} \mathrm{Ps} \rightarrow \mathrm{He} e^{+}$ & \\
\hline
\end{tabular}


Fig. 1 - Schematic representation of the processes by which positrons annihilate.

Fig. 2 - Cross sections for ionization (ion), excitation (ex) and charge exchange (ce) interactions of positrons with $\mathrm{H}$ (solid) and He (dashed). Also shown is the cross section for direct annihilation of positrons with free electrons (daf; dotted), multiplied by $10^{5}$.

Fig. 3 - Cross sections for quenching interactions of Ps. If the interaction is with an atom or ion then the abscissa gives the Ps energy. If the interaction is with an electron then the abscissa gives the energy of the electron in the rest frame of the Ps.

Fig. 4 - The fraction of positrons which form Ps in flight as a function of the ionization fraction of $\mathrm{H}\left(X_{\mathrm{H}^{+}}\right)$for $n_{e}=5 \times 10^{13} \mathrm{~cm}^{-3}$ and $T=11,600 \mathrm{~K}$. The various curves are discussed in the text.

Fig. 5 - The Ps energy distribution resulting from formation in flight by primary positrons calculated by the Monte Carlo simulation for $X_{\mathrm{H}^{+}}=0$ (solid) and $X_{\mathrm{H}^{+}}=0.05$ (dotted). The dashed histogram show the distribution for secondary positrons resulting from ionization quenching of primary ${ }^{3} \operatorname{Ps}\left(X_{\mathrm{H}^{+}}=0\right)$.

Fig. 6 - Thermally averaged rates per unit target density for direct annihilation (daf) and radiative combination ( $\mathrm{rc}$ ) with free electrons, charge exchange (ce) with $\mathrm{H}$ and $\mathrm{He}$ and direct annihilation with bound electrons (dab) of $\mathrm{H}$ and He. The curve for direct annihilation with bound electrons of $\mathrm{H}$ ends at $T \simeq 10^{4} \mathrm{~K}$ since above this temperature, $\mathrm{H}$ is considered totally ionized.

Fig. 7 - Thermally averaged quenching rates calculated from the cross sections of Fig. 3. The Ps has the velocity distribution resulting from Ps formation through thermal radiative combination in panel (a), thermal charge exchange with $\mathrm{H}$ in panel (b), thermal charge exchange with He in panel (c), and charge exchange in flight in panel (d). We note that at the higher temperatures, neutral $\mathrm{H}$ or He may not be present fo charge exchange to occur.

Fig. 8 - Line profiles for the $2 \gamma$ annihilation of ${ }^{1} \mathrm{Ps}$ formed through charge exchange of thermal positrons with $\mathrm{H}$ and $\mathrm{He}$ for several temperatures.

Fig. 9 - Line profile for the $2 \gamma$ annihilation of ${ }^{1}$ Ps formed through charge exchange in flight using the Ps energy distributions from primary positrons shown in Figure $5: X_{\mathrm{H}^{+}}=0$ (solid) and $X_{\mathrm{H}^{+}}=0.05$ (dotted). The dashed histogram shows the line profile using the Ps distribution from secondary positrons $\left(X_{\mathrm{H}^{+}}=0\right)$.

Fig. 10 - Ratio of the number of $3 \gamma$ continuum photons to $2 \gamma$ line photons $\left(Q_{3 \gamma} / Q_{2 \gamma}\right)$ as a function of temperature for various densities in a fully ionized medium. 
Fig. 11 - Ratio of the number of $3 \gamma$ continuum photons to $2 \gamma$ line photons $\left(Q_{3 \gamma} / Q_{2 \gamma}\right)$ as a function of density for various temperatures in a fully ionized medium. The dashed curves are for a neutral medium at $T=4000,5000$ and $6000 \mathrm{~K}$.

Fig. 12 - Calculated line profiles for fully ionized medium at $T=10^{4}$ and $10^{6} \mathrm{~K}$ with $n_{\mathrm{H}}=10^{13} \mathrm{~cm}^{-3}$. The components contributing to the spectrum are indicated: radiative combination with free electrons (rc), direct annihilation with free electrons (daf) and the $3 \gamma$ ${ }^{3}$ Ps continuum ( $3 \gamma$, dotted curve). The normalization is 1 initial positron.

Fig. 13 - Ratio of the number of $3 \gamma$ continuum photons to $2 \gamma$ line photons $\left(Q_{3 \gamma} / Q_{2 \gamma}\right)$ as a function of line width for various densities in a fully ionized medium. Because in a fully ionized medium the width is directly related to the temperature through equation (15), we show the corresponding temperature scale along the top of the figure.

Fig. 14 - Calculated line profiles for a neutral medium at $n_{\mathrm{H}}=10^{9}$ and $10^{17} \mathrm{~cm}^{-3}$ with $T=5000 \mathrm{~K}$. The components contributing to the spectrum are indicated: in-flight charge exchange (ifce), direct annihilation with bound $\mathrm{H}$ electrons (dab, $\mathrm{H}$ ), direct annihilation with bound He electrons (dab,He), thermal charge exchange with $\mathrm{H}(\mathrm{ce}, \mathrm{H})$, second-generation in-flight charge exchange $(2 \mathrm{~g})$, and $3 \gamma^{3} \mathrm{Ps}$ continuum ( $3 \gamma$, dotted curve). The normalization is 1 initial positron.

Fig. 15 - Ratio of the number of $3 \gamma$ continuum photons to $2 \gamma$ line photons $\left(Q_{3 \gamma} / Q_{2 \gamma}\right)$ in a neutral medium as a function of the broad/narrow line ratio $\mathrm{R}_{\mathrm{b} / \mathrm{n}}$ for $T=4000,5000 \&$ $6000 \mathrm{~K}$ and various densities as indicated along each curve

Fig. 16 - Calculated line profiles for a partially-ionized medium with $T=5000 \mathrm{~K}, n_{\mathrm{H}}=$ $10^{15} \mathrm{~cm}^{-3}$, and $X_{\mathrm{H}^{+}}=0.0,0.01,0.10$ and 0.50 . The inset table gives the associated values of $f_{1}, Q_{3 \gamma} / Q_{2 \gamma}$, formal line width (FWHM), and the broad/narrow line ratio $\mathrm{R}_{\mathrm{b} / \mathrm{n}}$. The normalization is to 1 initial positron.

Fig. 17 - The total $\mathrm{H}\left(\mathrm{H}^{0}+\mathrm{H}^{+}\right)$and electron densities versus temperature in the solar atmosphere according to model C of Vernazza et al. (1981). The numbers along the curve correspond to regions discussed in the text.

Fig. 18 - Ratio of the number of $3 \gamma$ continuum photons to $2 \gamma$ line photons $\left(Q_{3 \gamma} / Q_{2 \gamma}\right)$ as a function of temperature with the density and ionization fractions constrained by model $\mathrm{C}$ of Vernazza et al. (1981). The numbers along the curve correspond to the regions noted in Figure 17.

Fig. 19 - Ratio of the number of $3 \gamma$ continuum photons to $2 \gamma$ line photons $\left(Q_{3 \gamma} / Q_{2 \gamma}\right)$ as a function of density with the temperature and ionization fractions constrained by model $\mathrm{C}$ of Vernazza et al. (1981). The numbers along the curve correspond to the regions noted in 
Figure 17.

Fig. 20 - Line profiles for various temperatures with the density and ionization fractions constrained by model C of Vernazza et al. (1981). The annihilation modes are indicated: in-flight charge exchange (ifce), thermal charge exchange with $\mathrm{H}(\mathrm{ce}, \mathrm{H})$, thermal charge exchange with $\mathrm{He}(\mathrm{ce}, \mathrm{He})$, radiative combination with free electrons (rc), direct annihilation with bound $\mathrm{H}$ electrons (dab,H), direct annihilation with bound He electrons (dab,He), direct annihilation with free electrons (daf), second-generation in-flight charge exchange (2g), and $3 \gamma^{3} \mathrm{Ps}$ continuum ( $3 \gamma$, dotted curve). The normalization is to 1 initial positron.

Fig. 21 - Line FWHM and the broad/narrow line ratio $\mathrm{R}_{\mathrm{b} / \mathrm{n}}$ as functions of density with the temperature and ionization fractions constrained by model C of Vernazza et al. (1981). The numbers along the curve correspond to the regions noted in Figure 17. FWHM is on the left axis and $R_{b / n}$ is on the right.

Fig. 22 - Line FWHM and the broad/narrow line ratio $\mathrm{R}_{\mathrm{b} / \mathrm{n}}$ as functions of temperature with the density and ionization fractions constrained by model C of Vernazza et al. (1981). The numbers along the curve correspond to the regions noted in Figure 17. FWHM is on the left axis and $R_{b / n}$ is on the right.

Fig. 23 - Ratio of the number of $3 \gamma$ continuum photons to $2 \gamma$ line photons $\left(Q_{3 \gamma} / Q_{2 \gamma}\right)$ as a function of the line FWHM and the broad/narrow line ratio $R_{b / n}$ with the density and ionization fractions constrained by model C of Vernazza et al. (1981). The numbers along the curve correspond to the regions noted in Figure 17. FWHM is on the bottom axis and $\mathrm{R}_{\mathrm{b} / \mathrm{n}}$ is on the top.

Fig. 24 - Observed count spectra and fits for the 2003 November 2 and 2003 October 28 flares. The November 2 spectrum is shown in (panel (a), along with the $T=5000 \mathrm{~K}$ Vernazza fit. The October 28 broad line is shown in panel (b), overplotted with the $T=$ $6000 \mathrm{~K}$ Vernazza (solid curve) and Gaussian (dotted curve) fits. The October 28 narrow line is shown in panel (c), over plotted with the $20 \%$ ionized (solid curve) and the Gaussian (dotted curve) fits. 


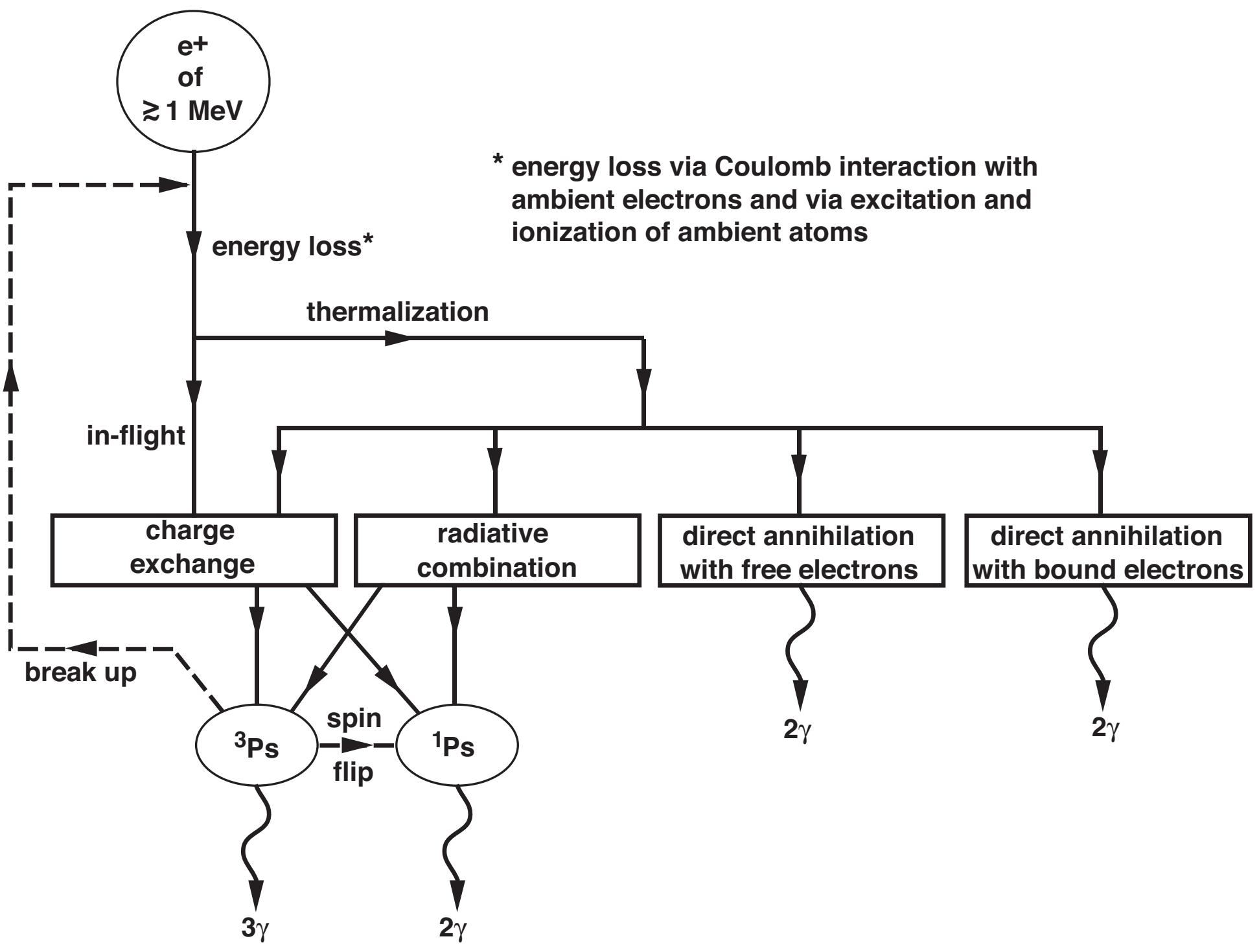

Figure 1 


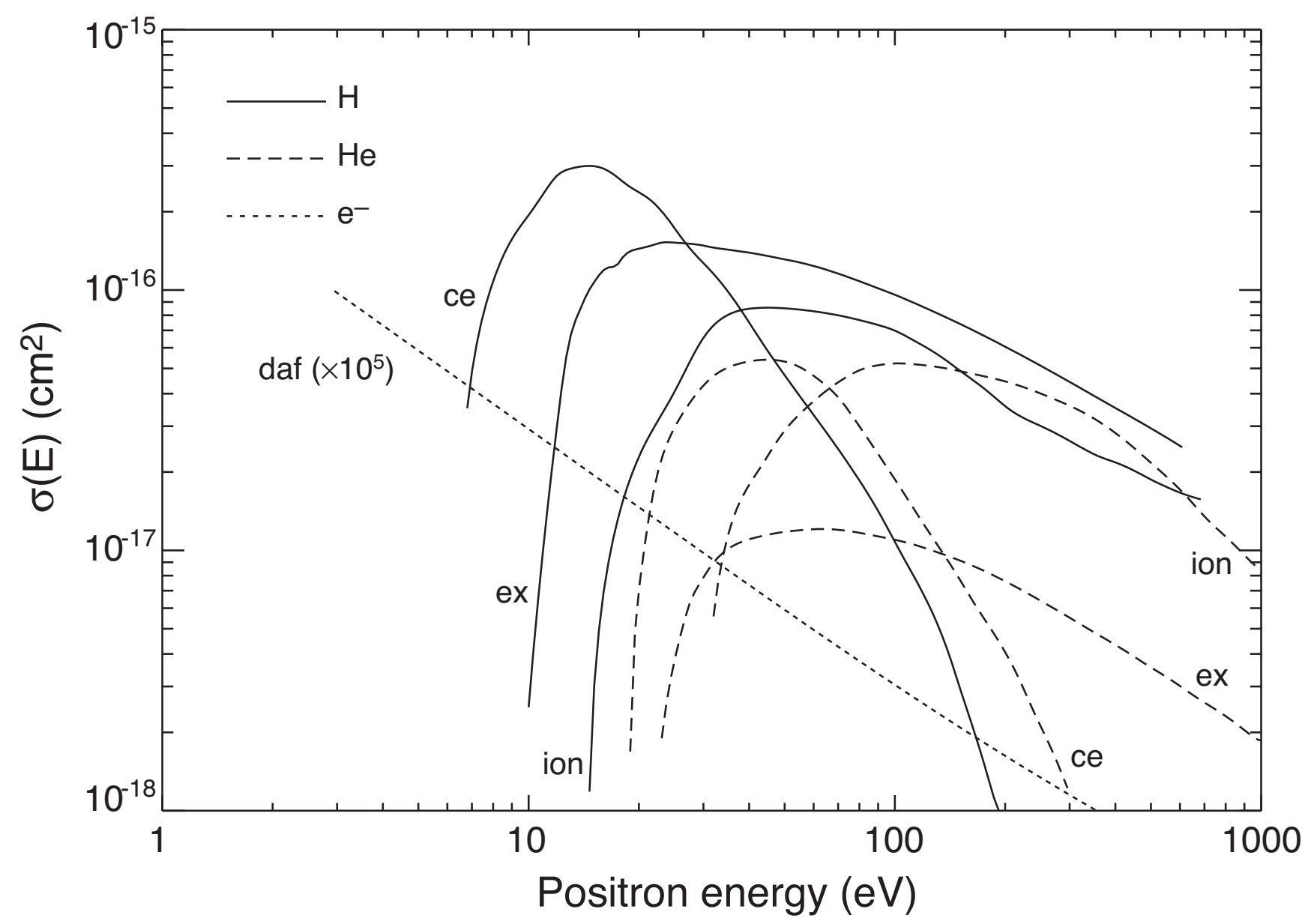

Figure 2 


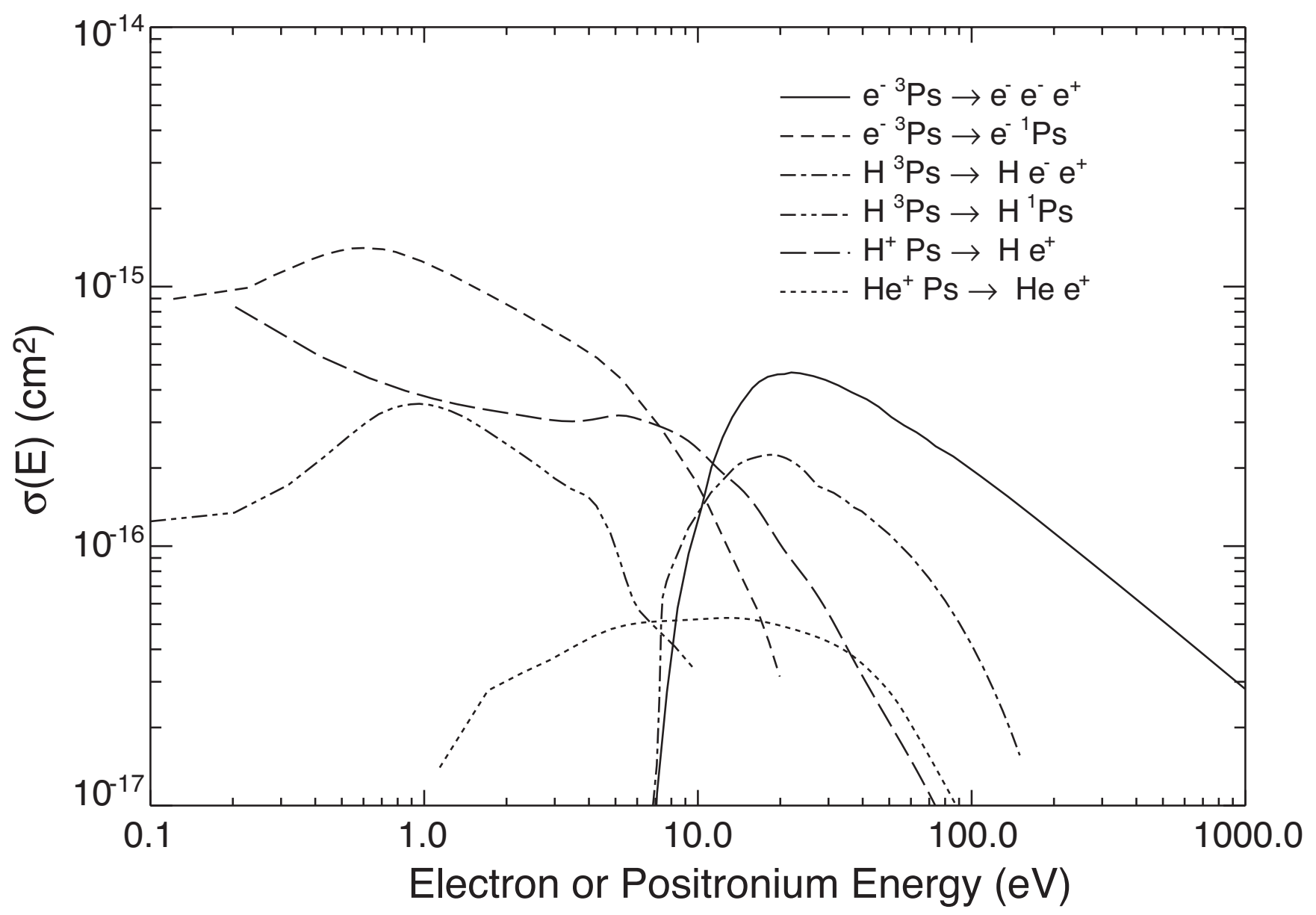

Figure 3 


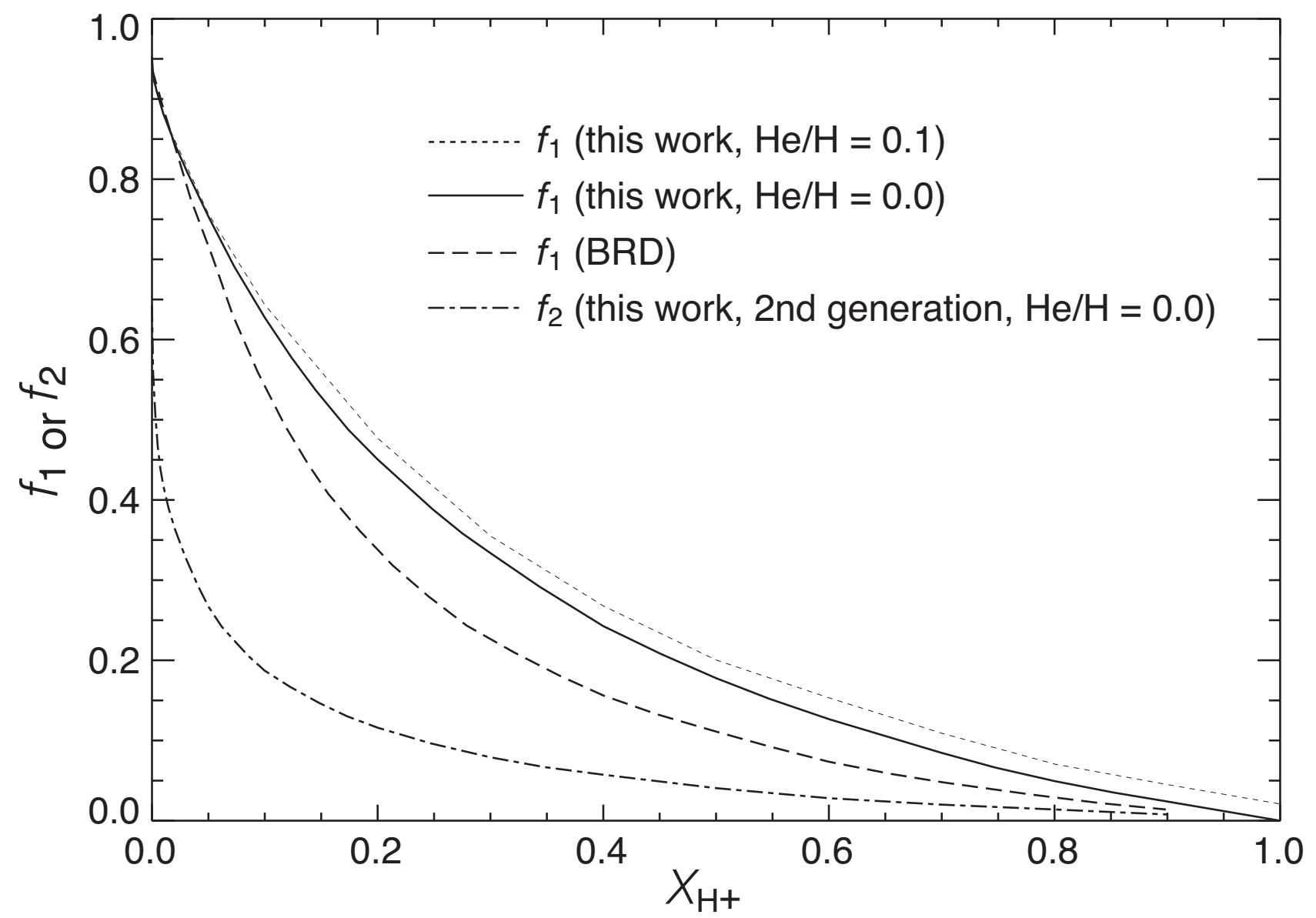

Figure 4 


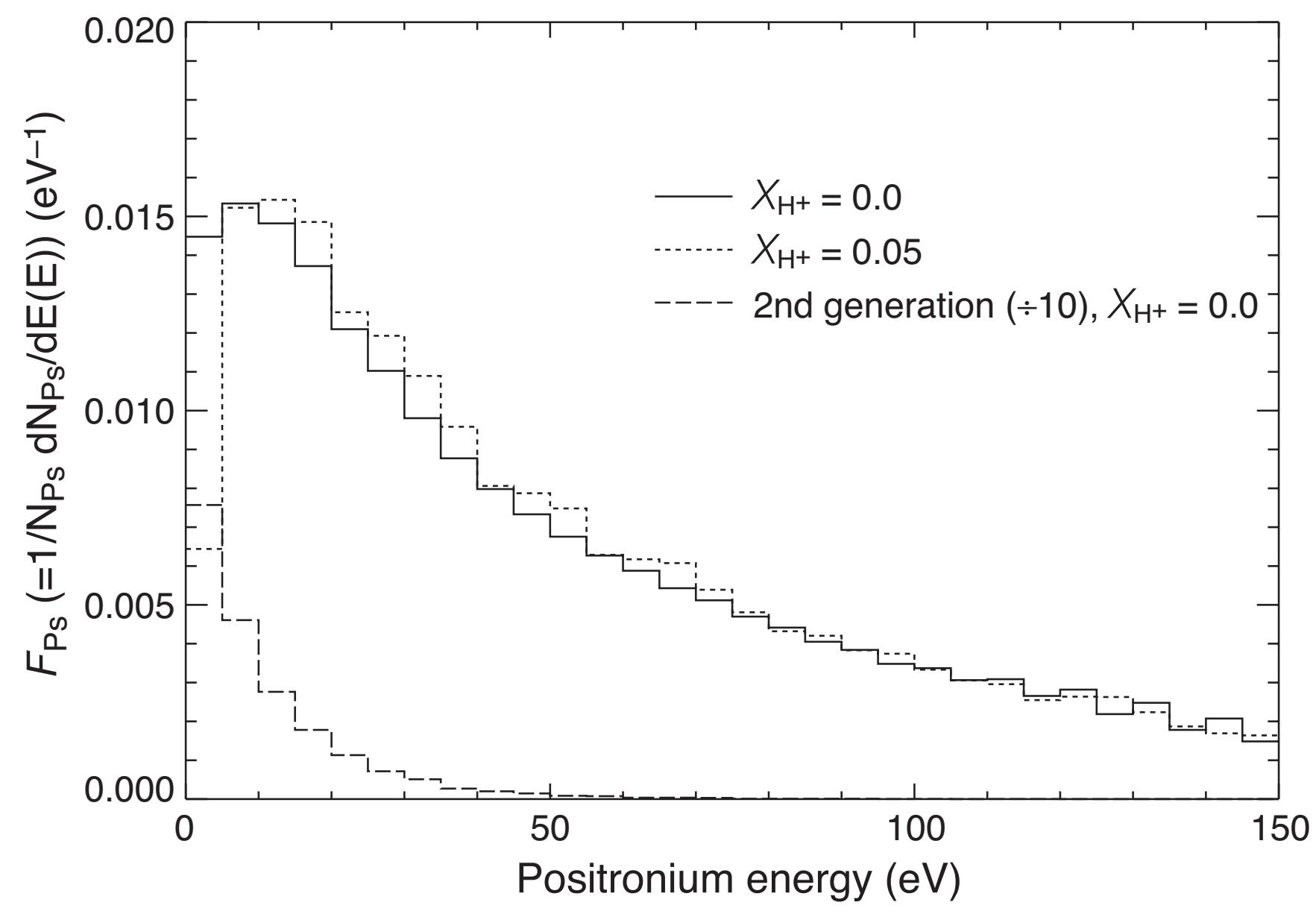

Figure 5 


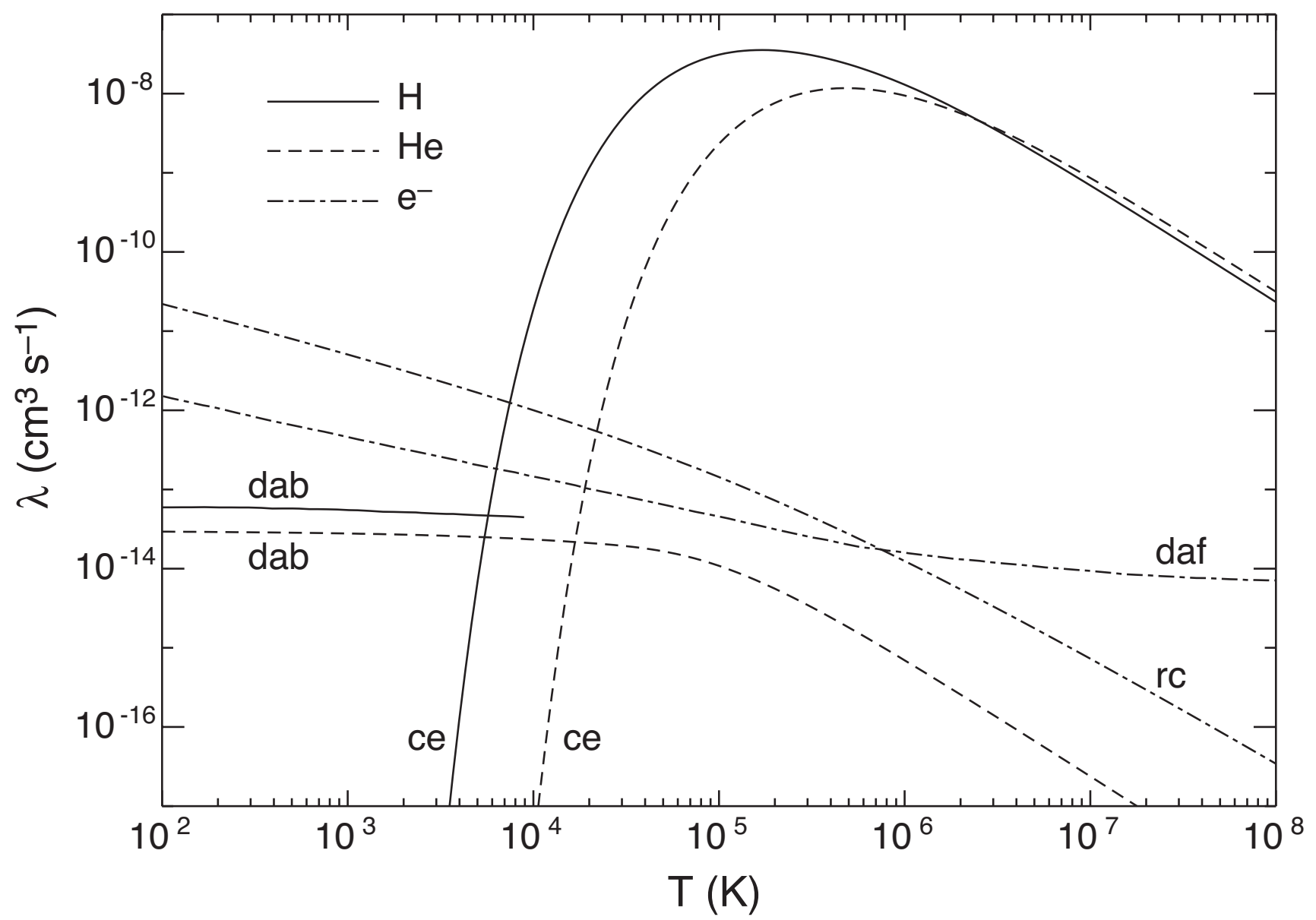

Figure 6 


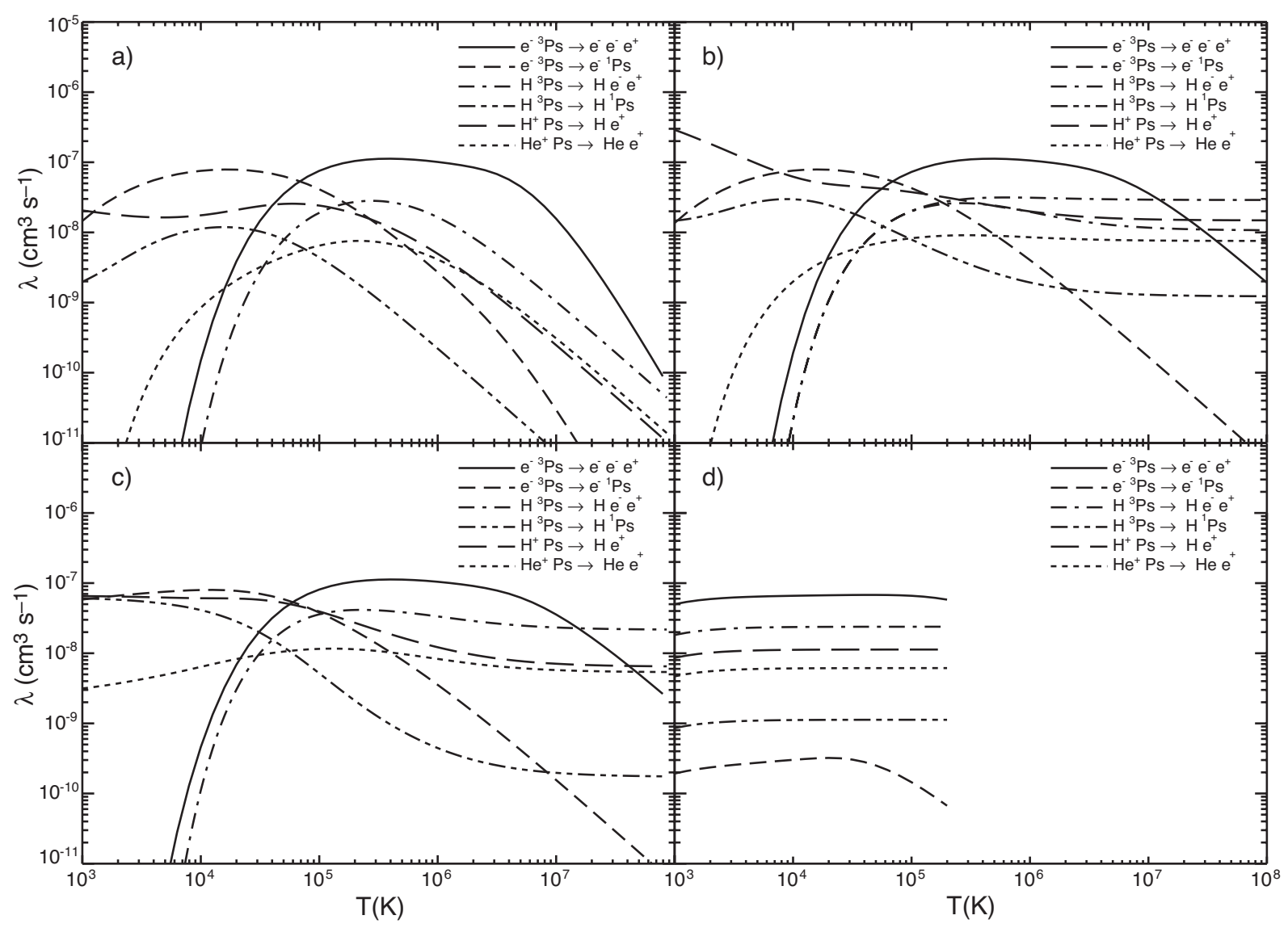

Figure 7 


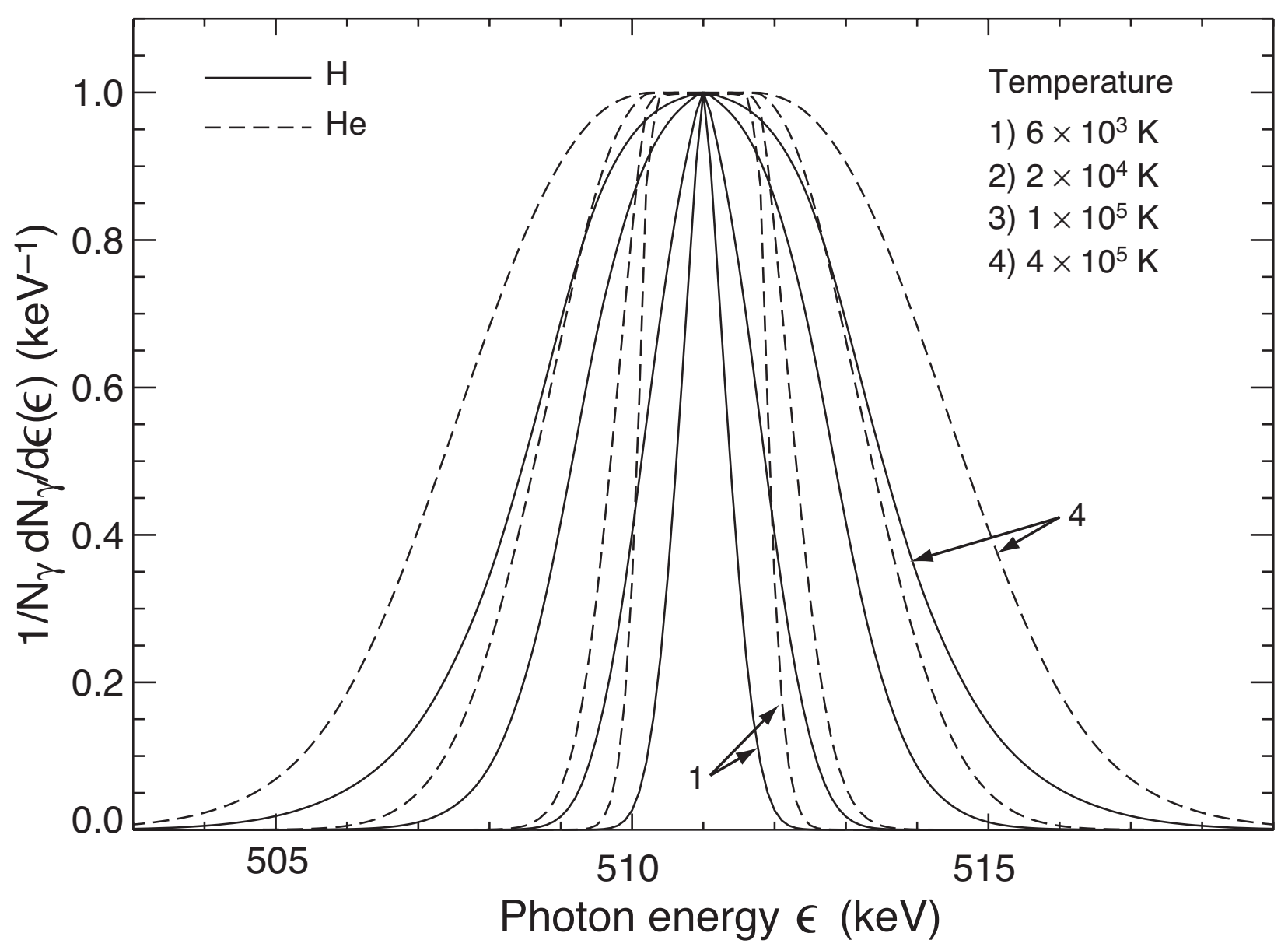

Figure 8 


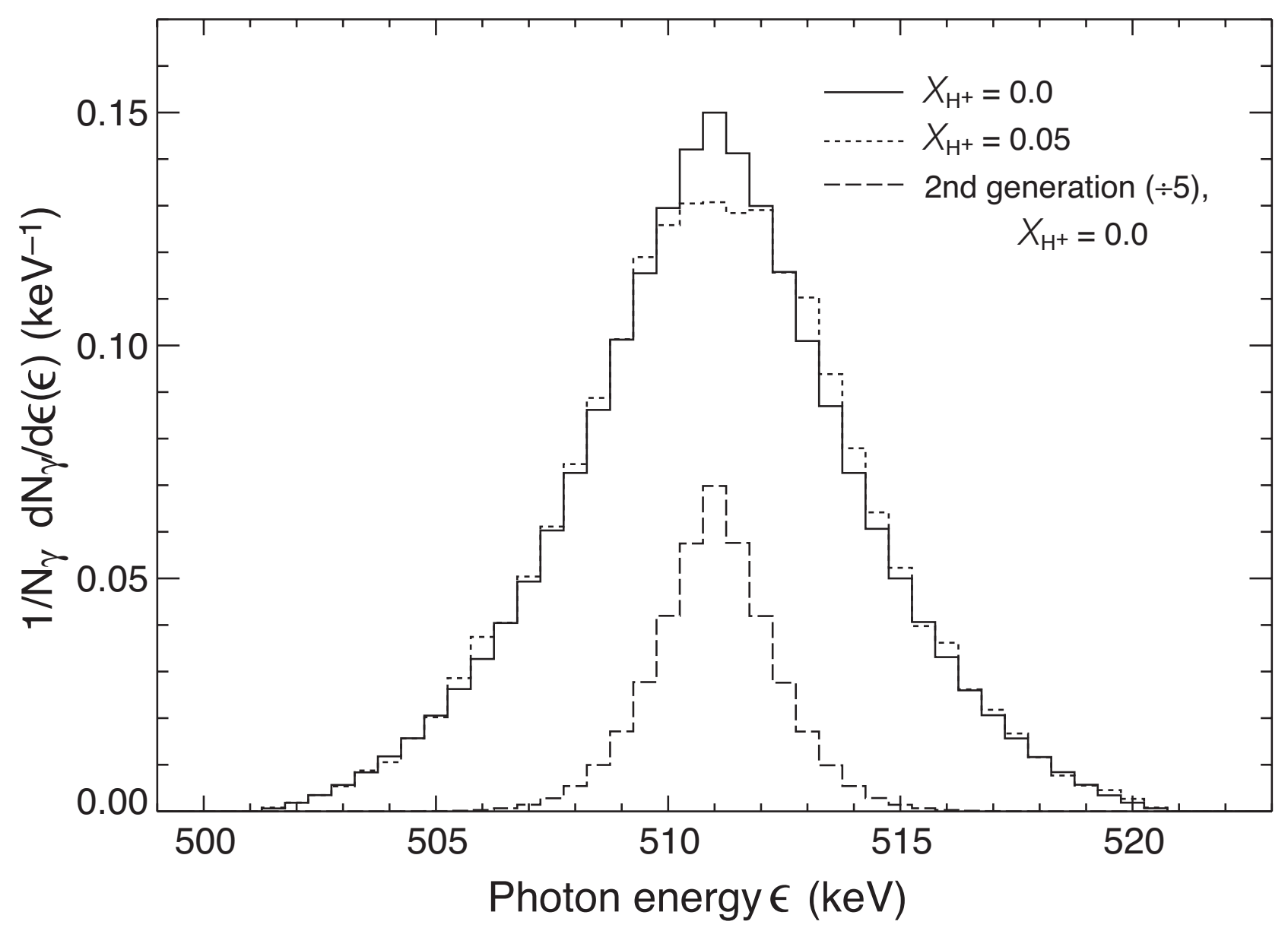

Figure 9 


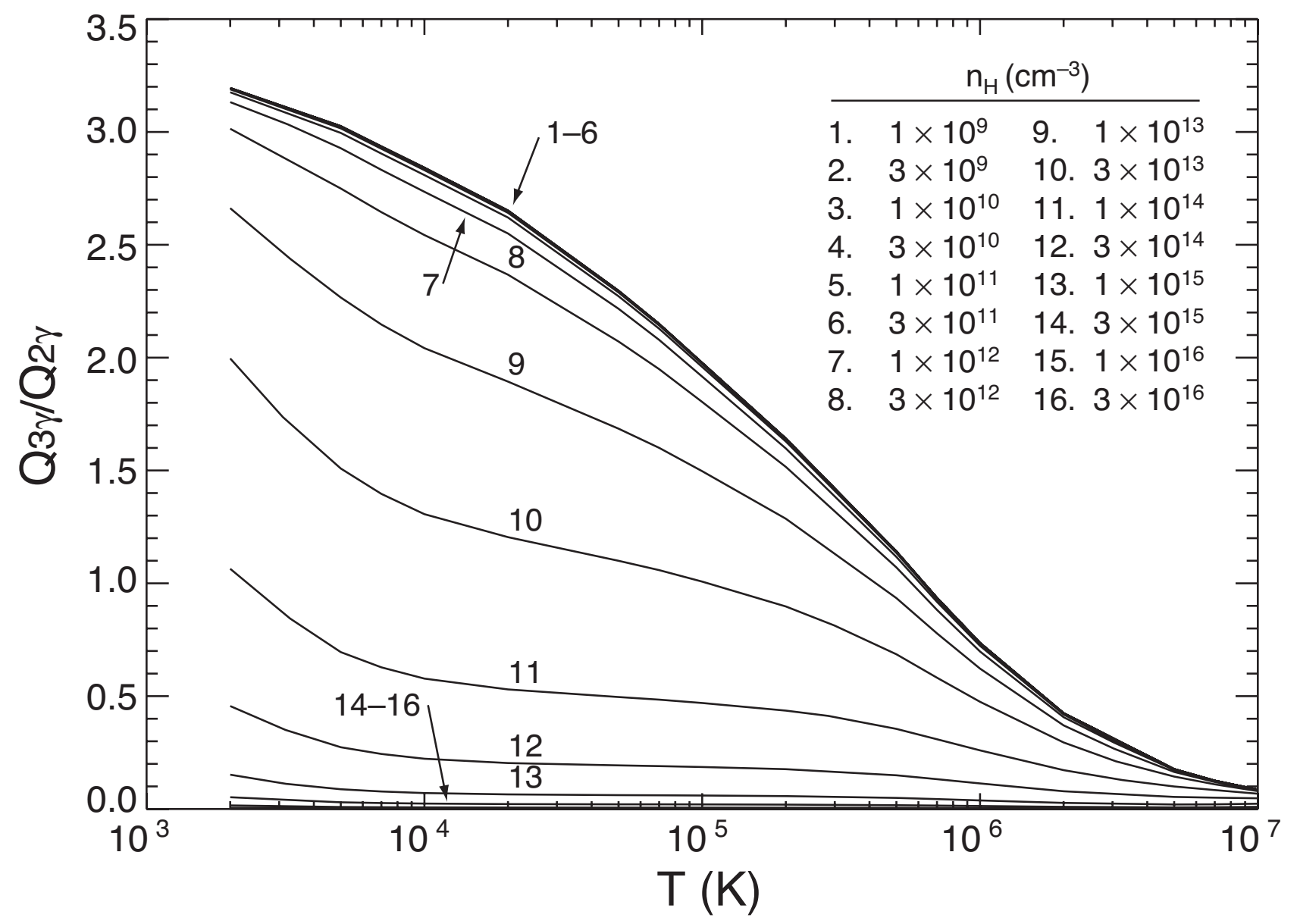

Figure 10 


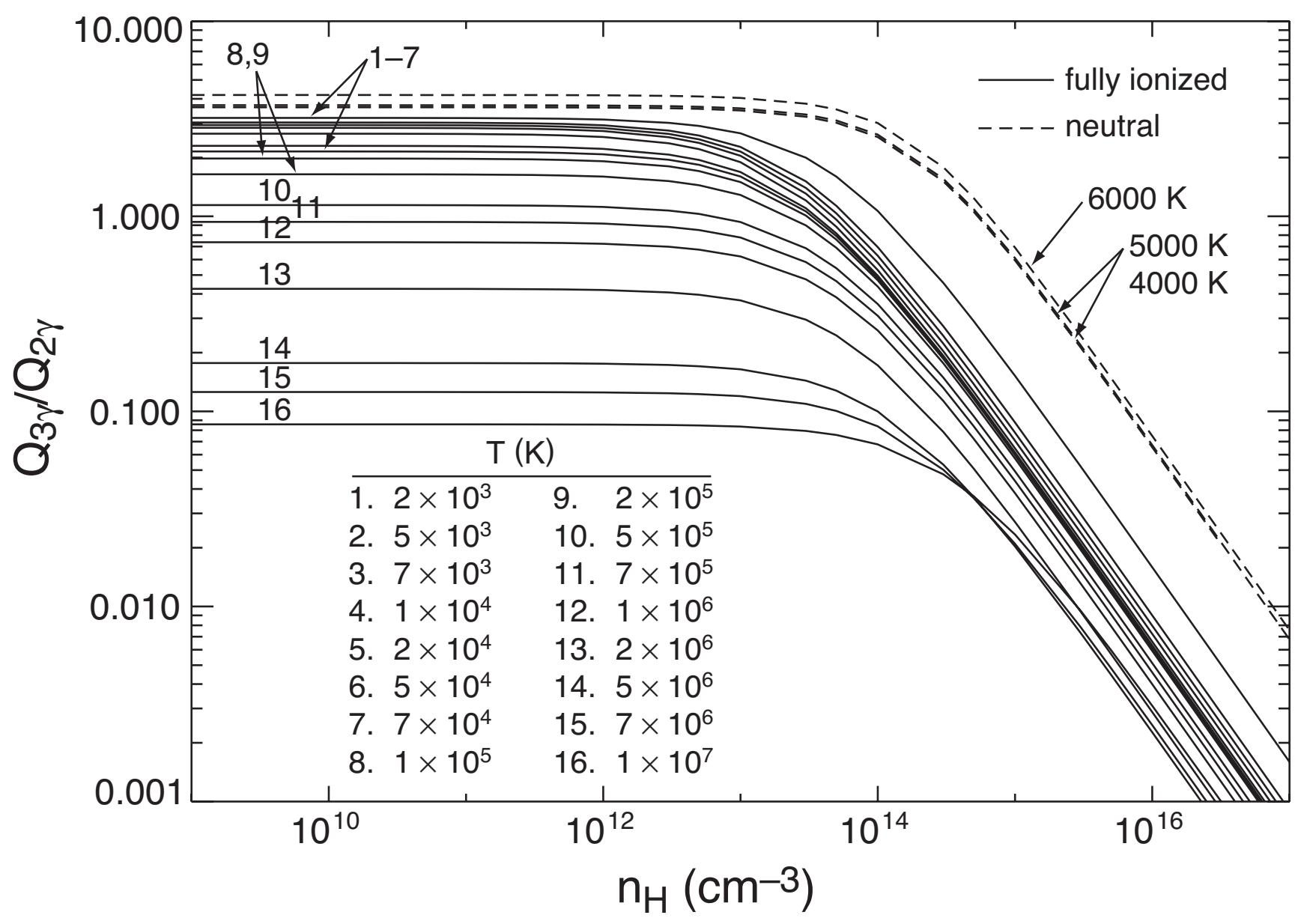

Figure 11 


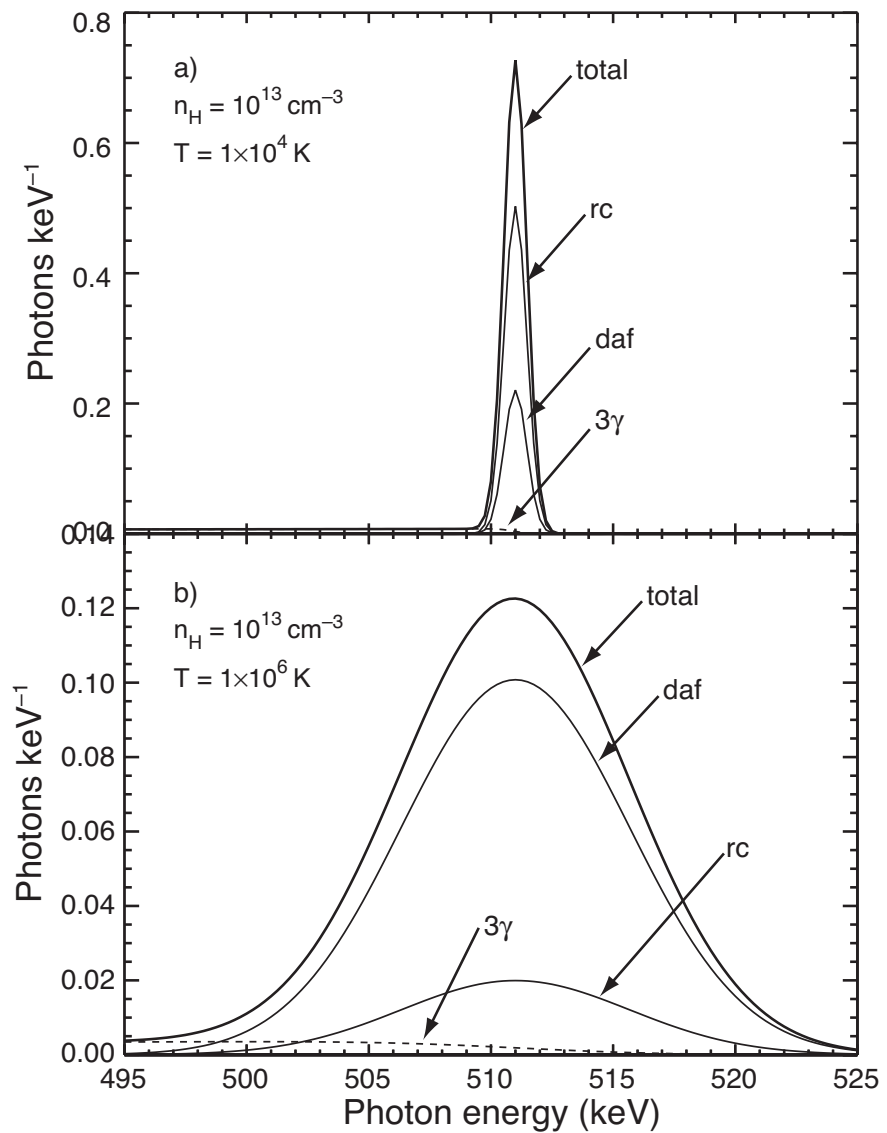

Figure 12 


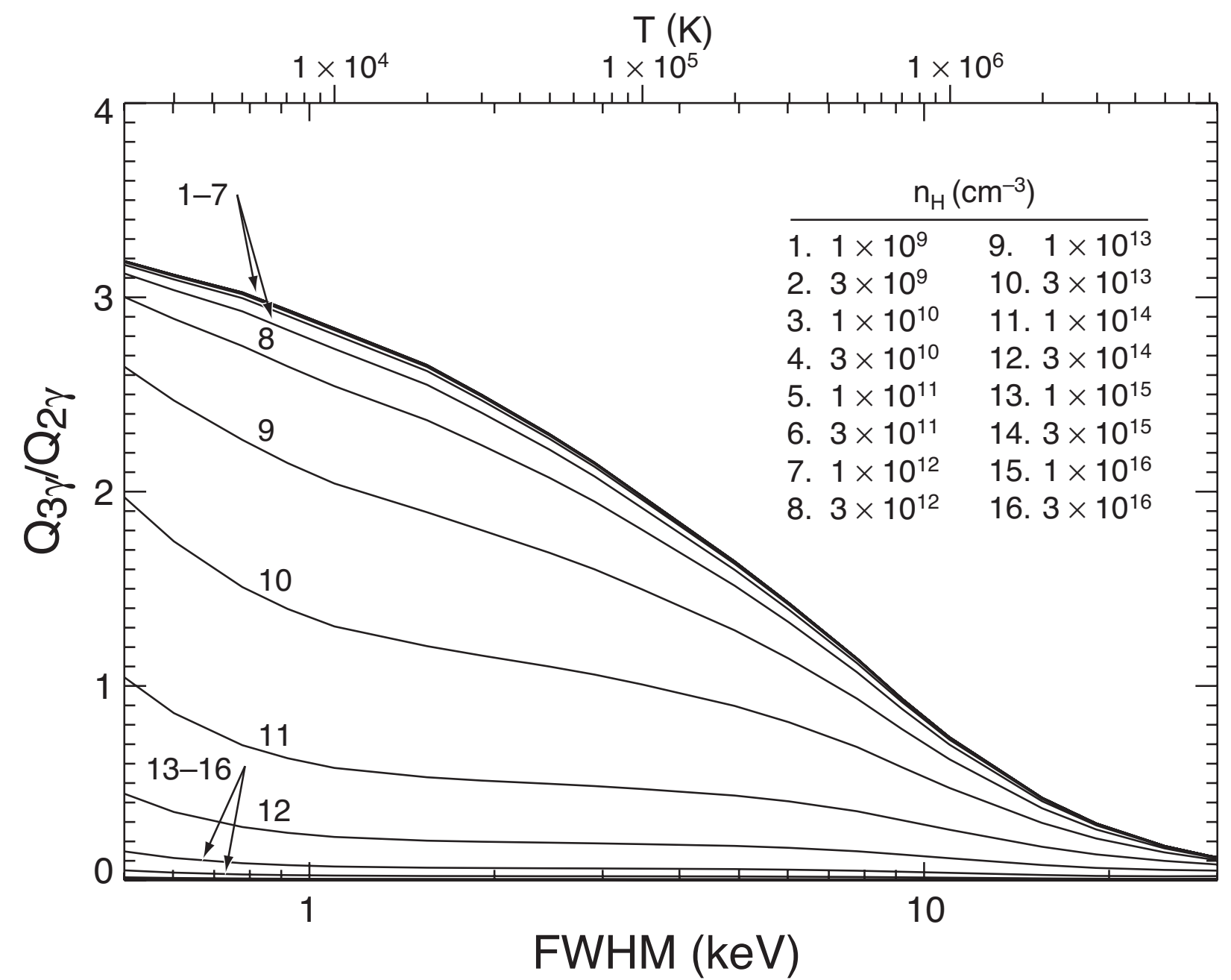

Figure 13 


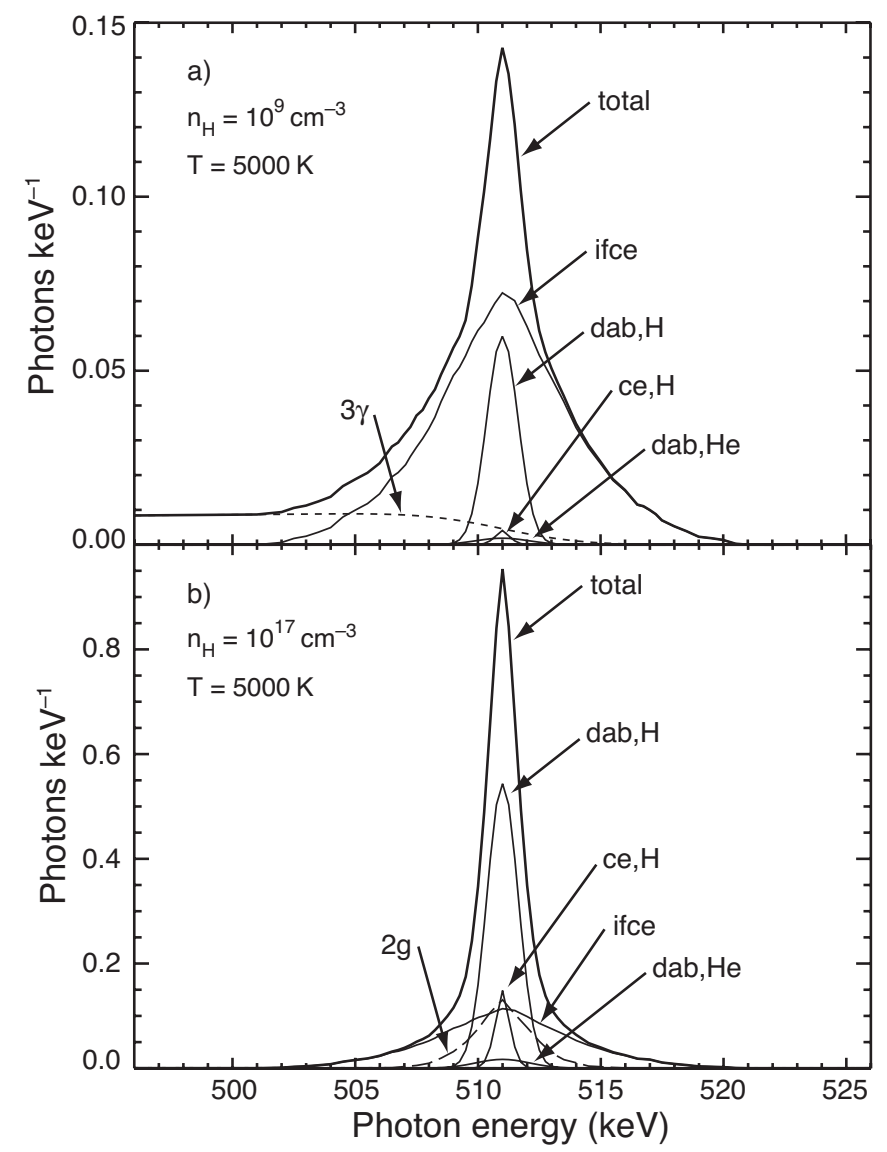

Figure 14 


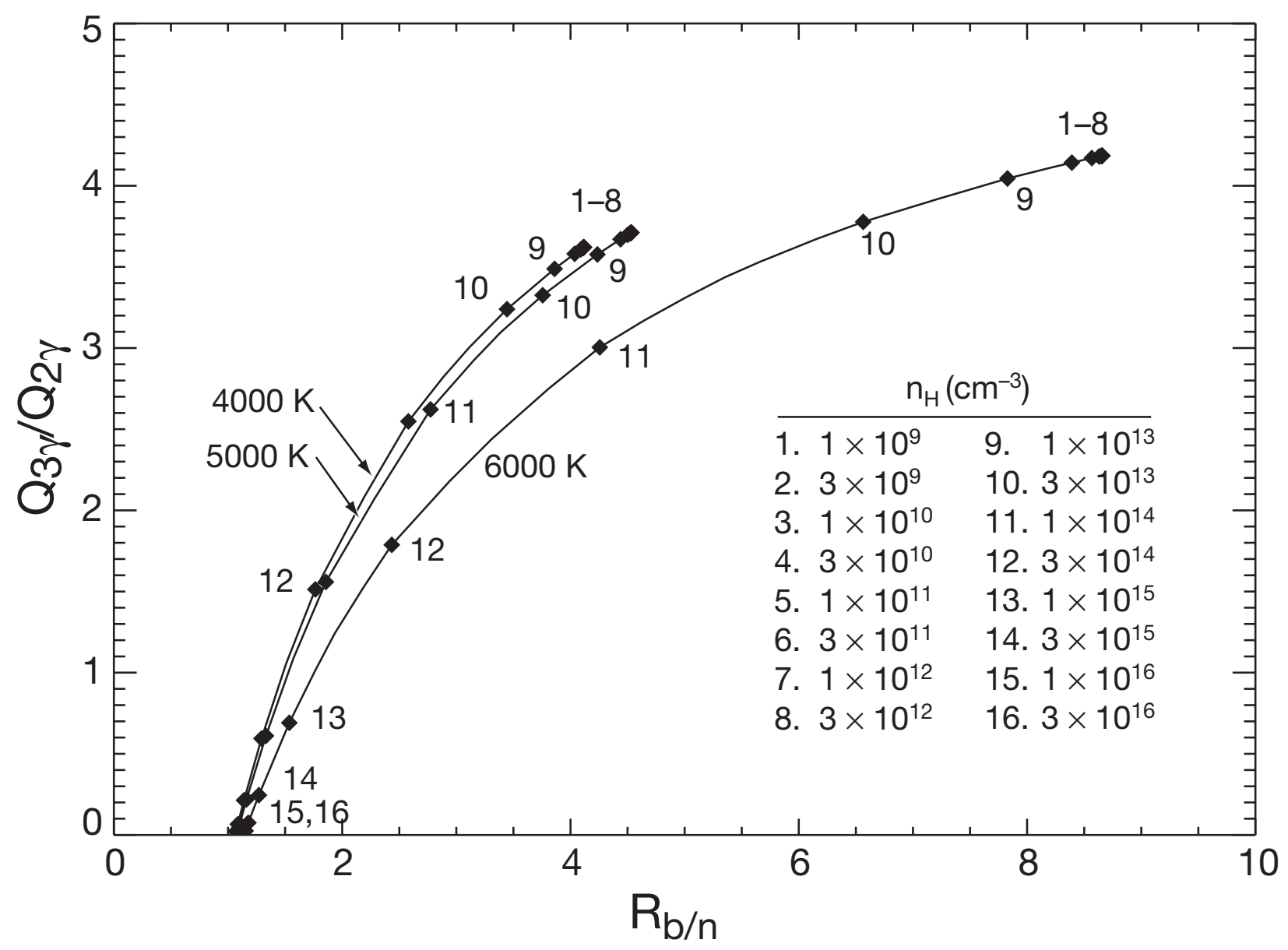

Figure 15 


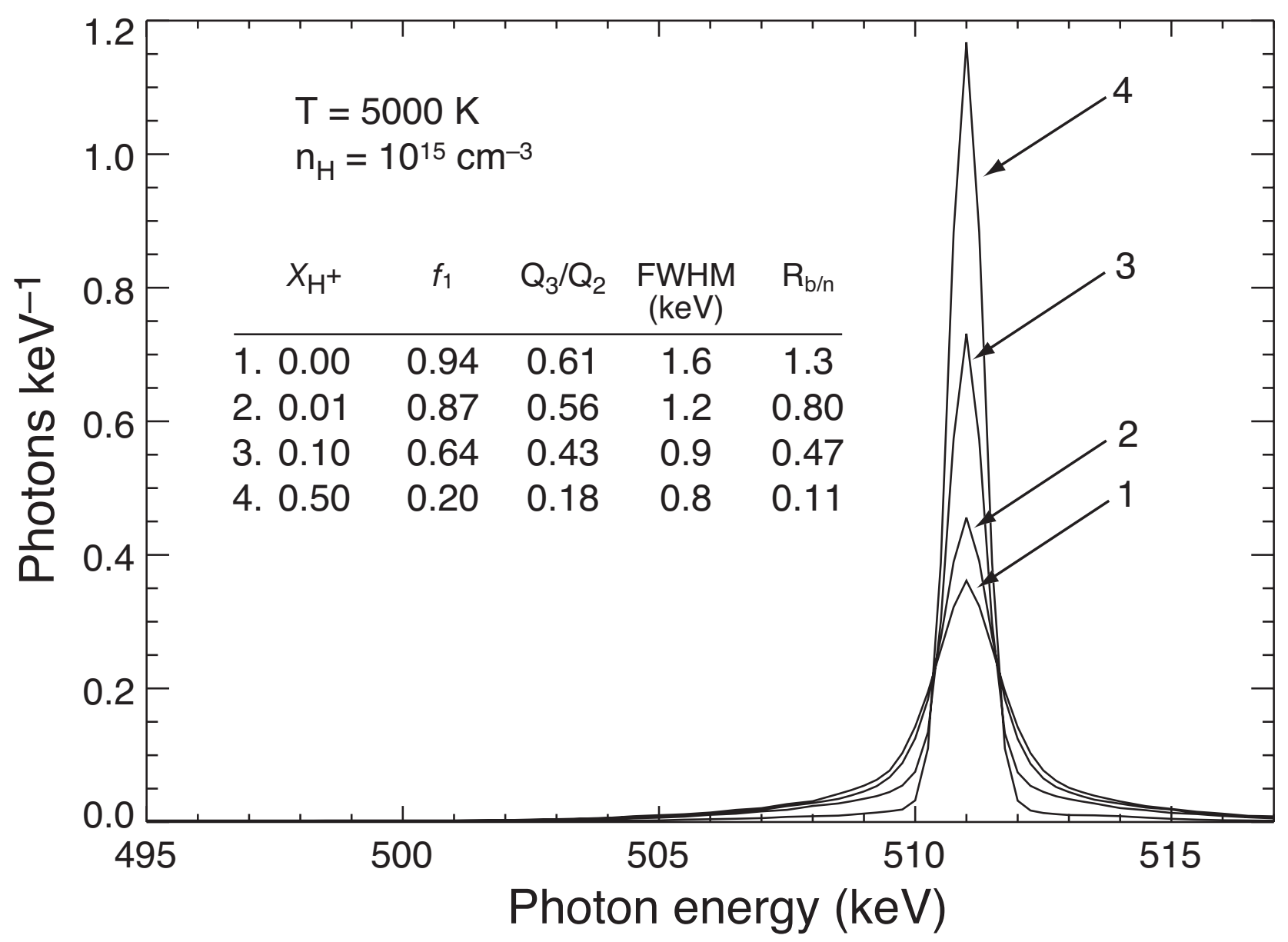

Figure 16 


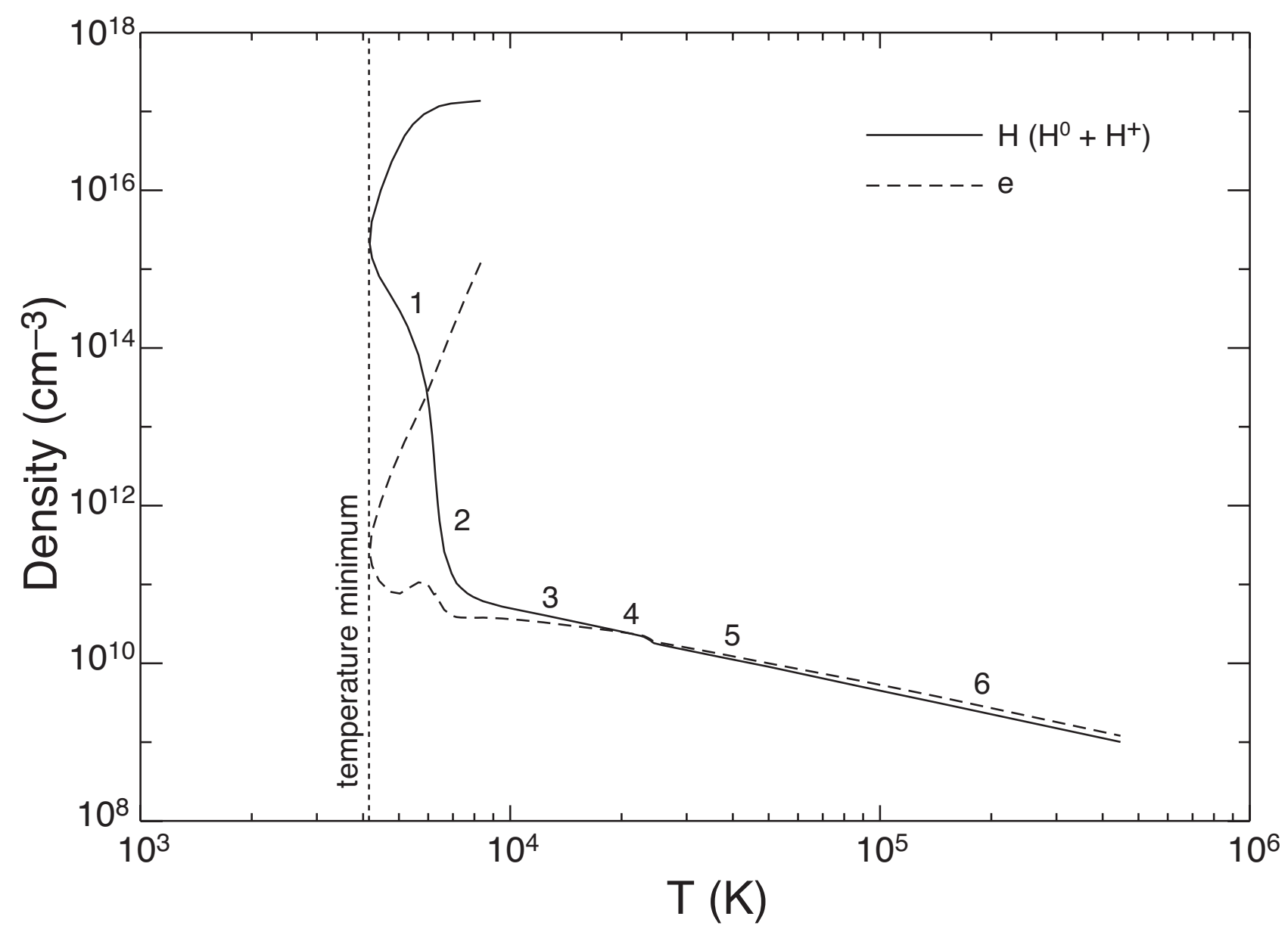

Figure 17 


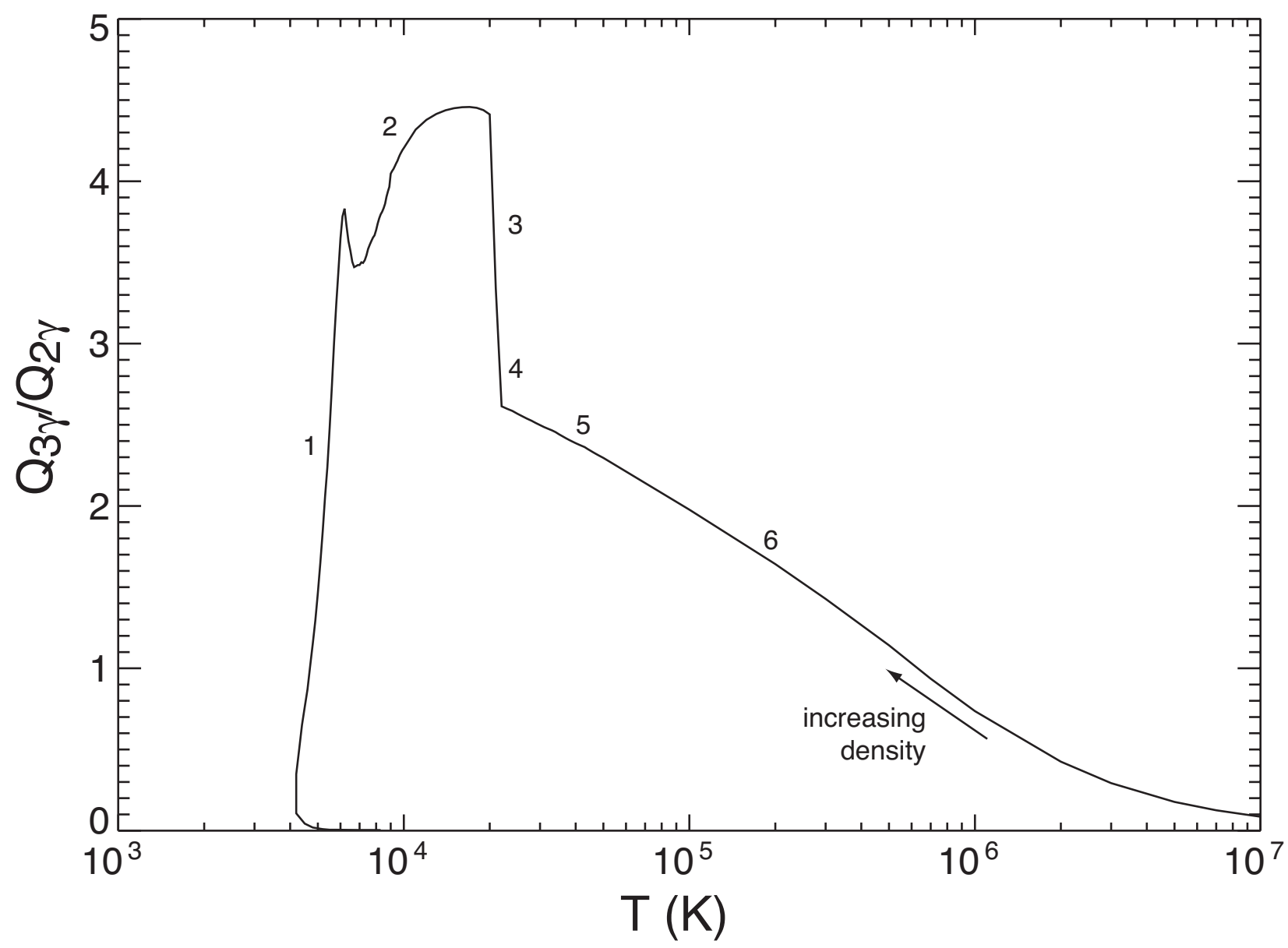

Figure 18 


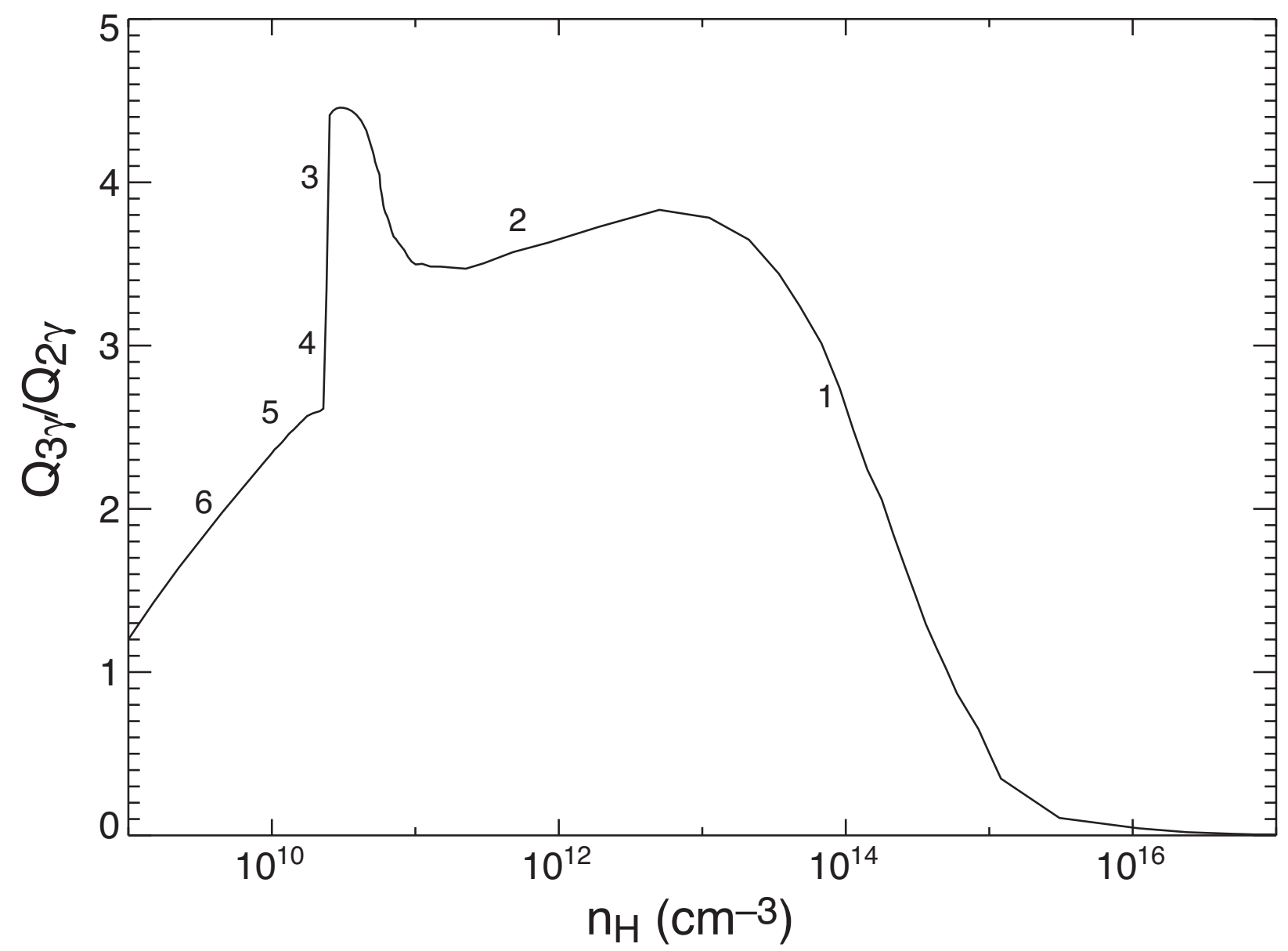

Figure 19 

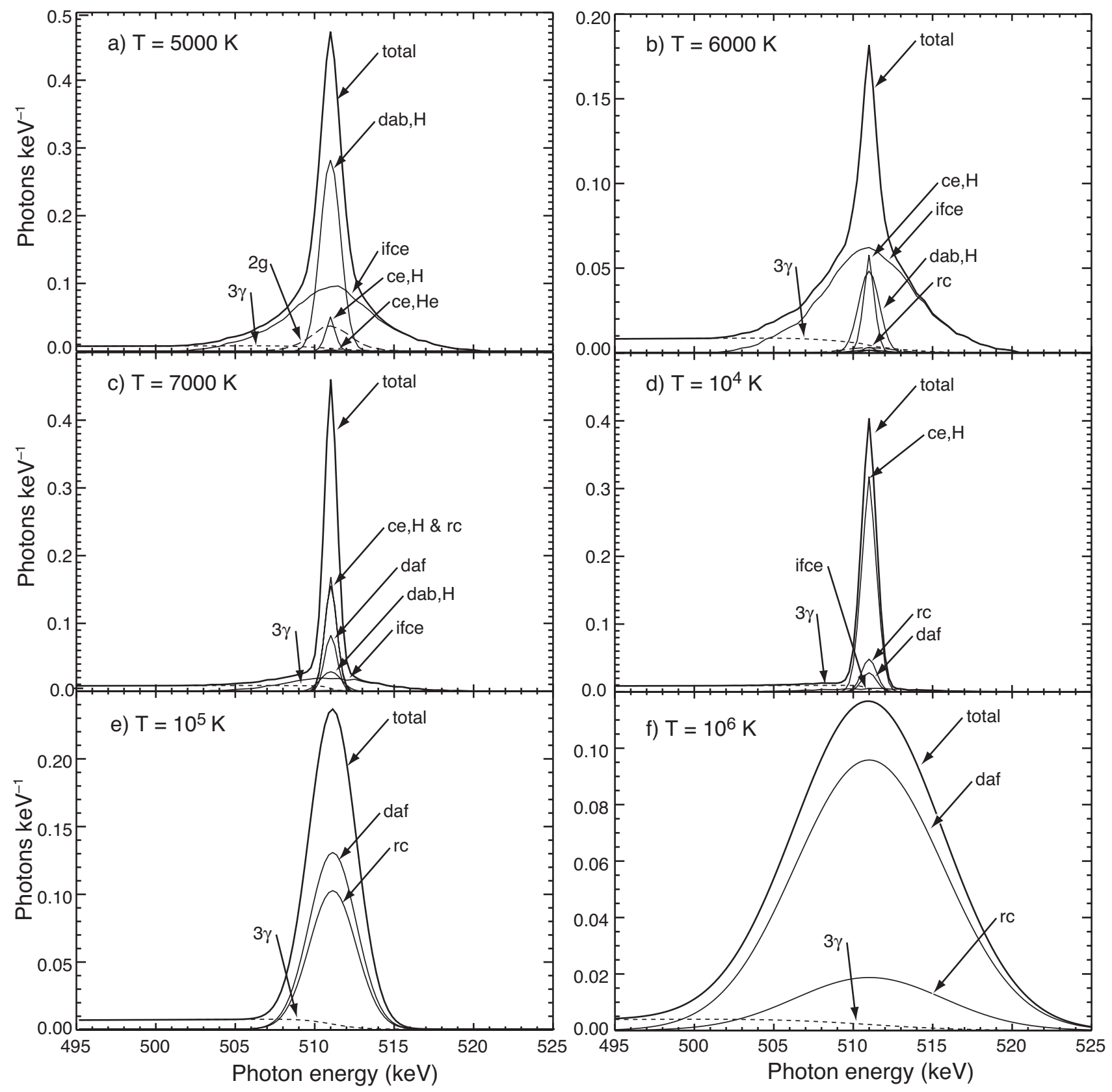

Figure 20 


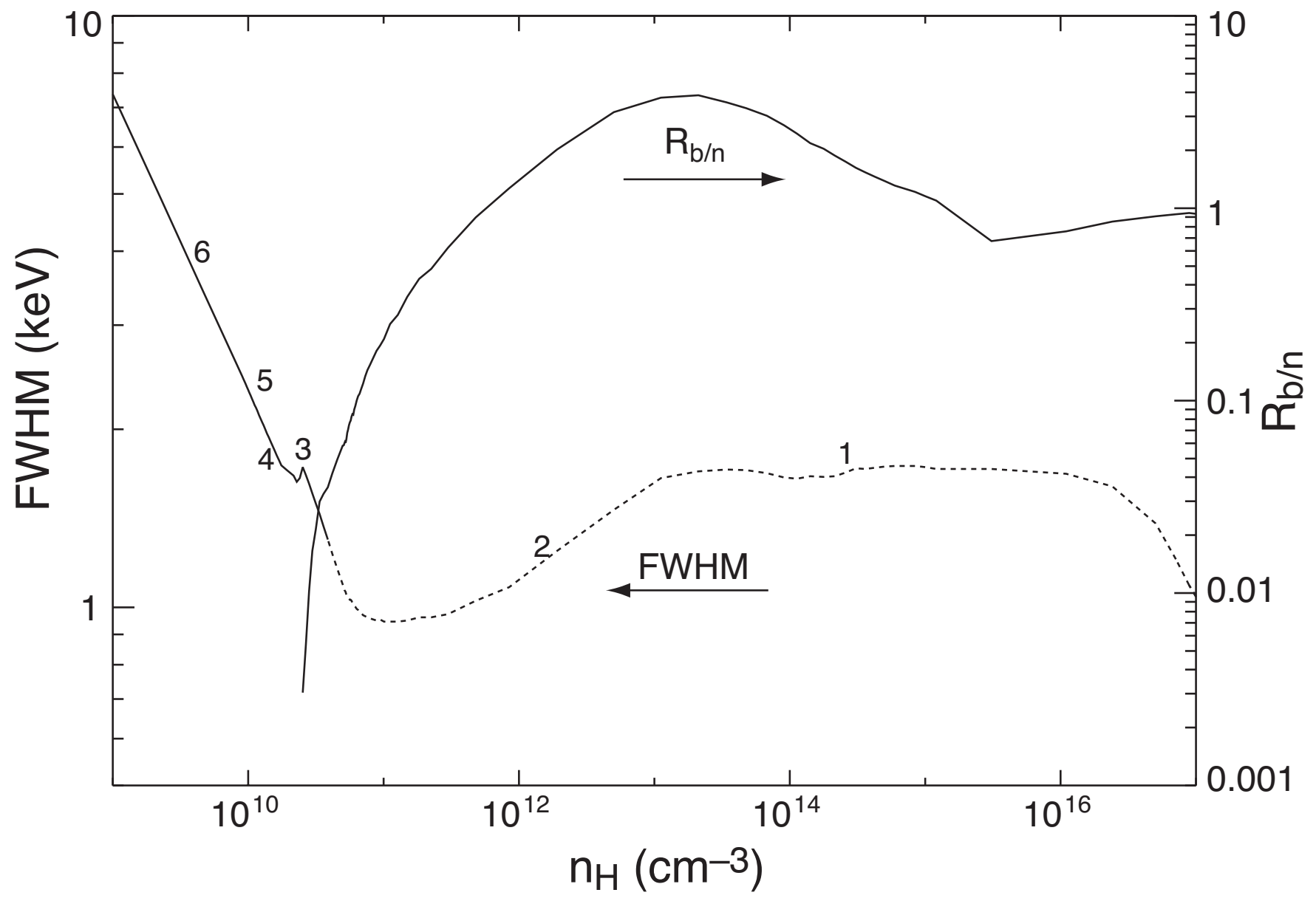

Figure 21 


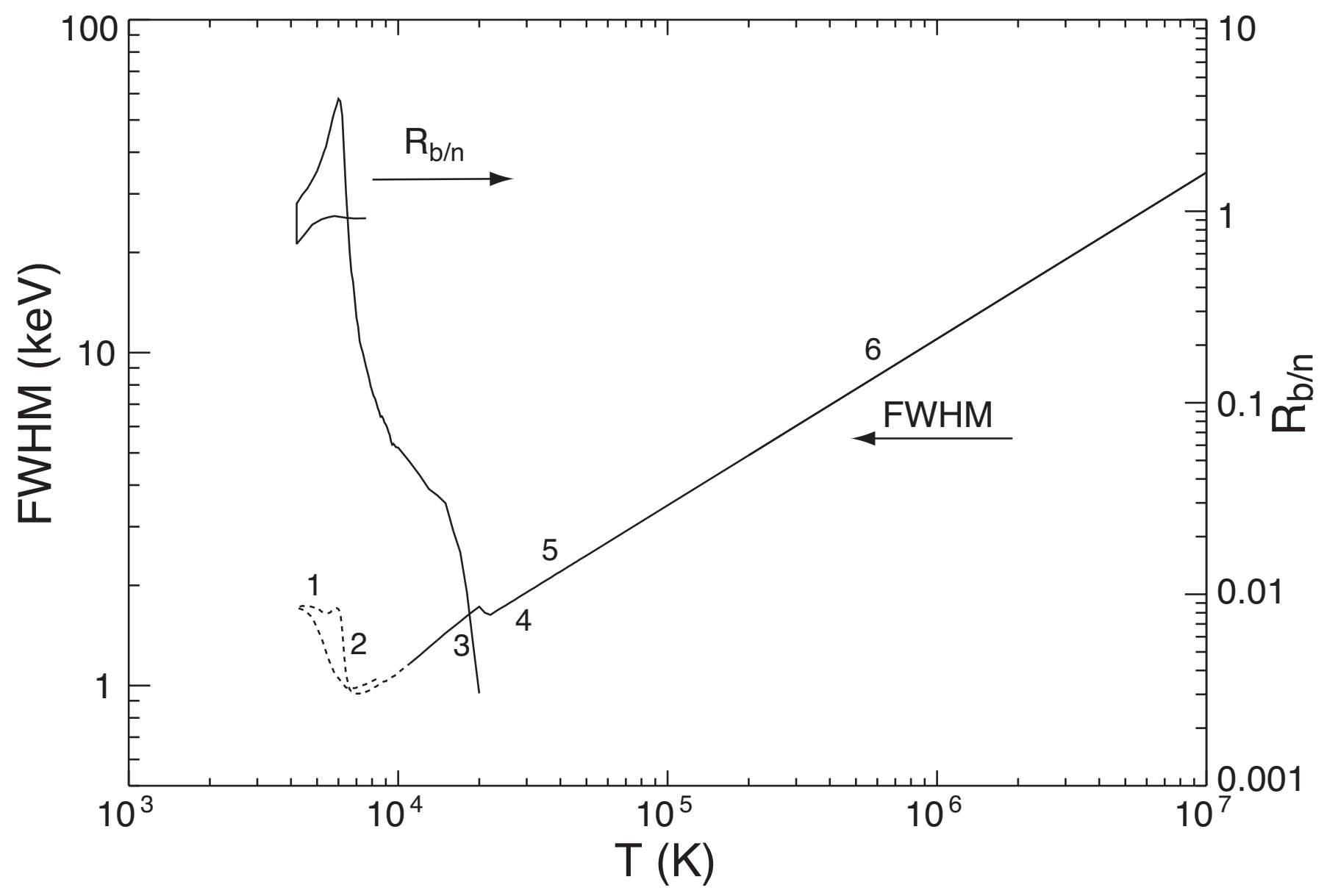

Figure 22 


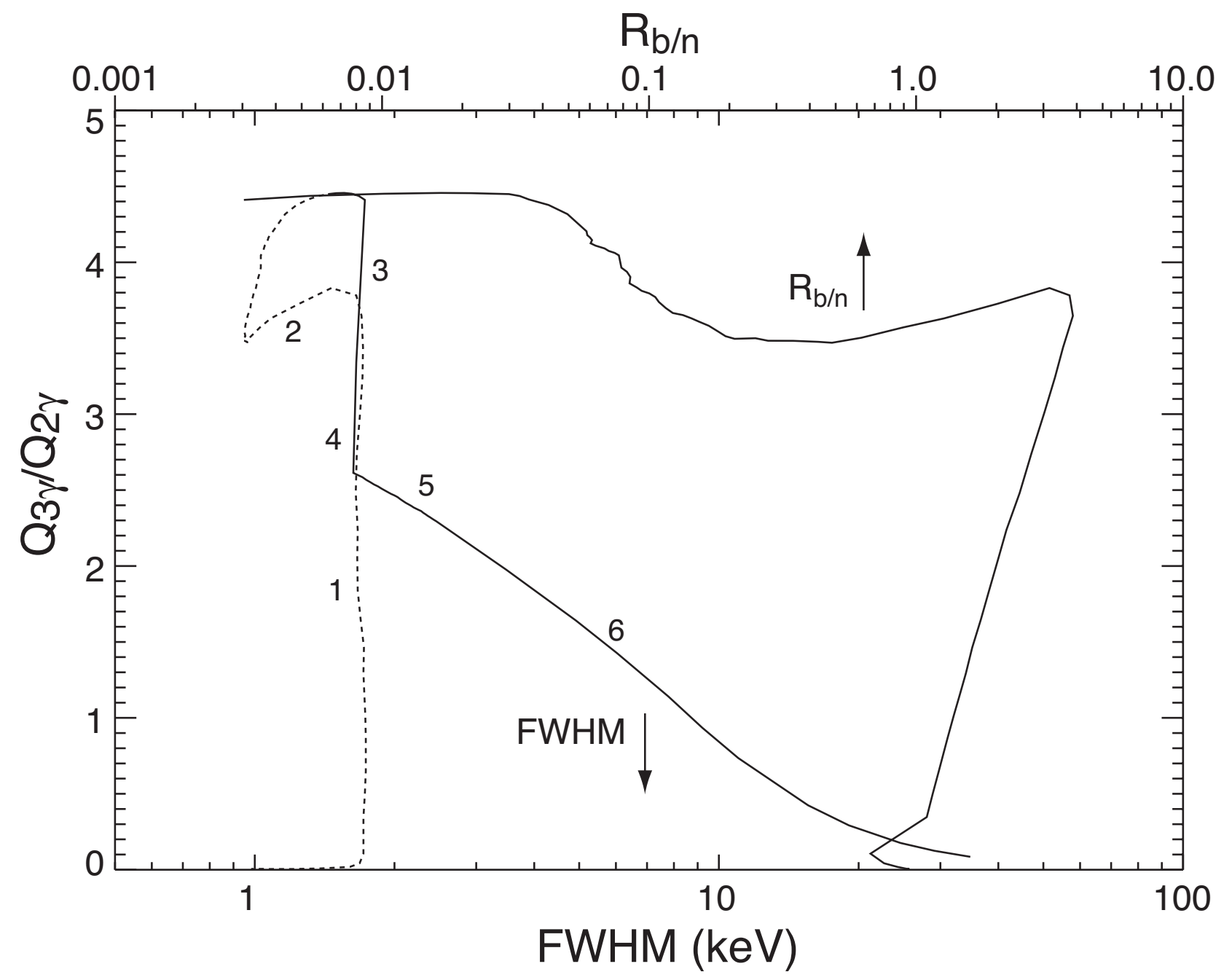

Figure 23 


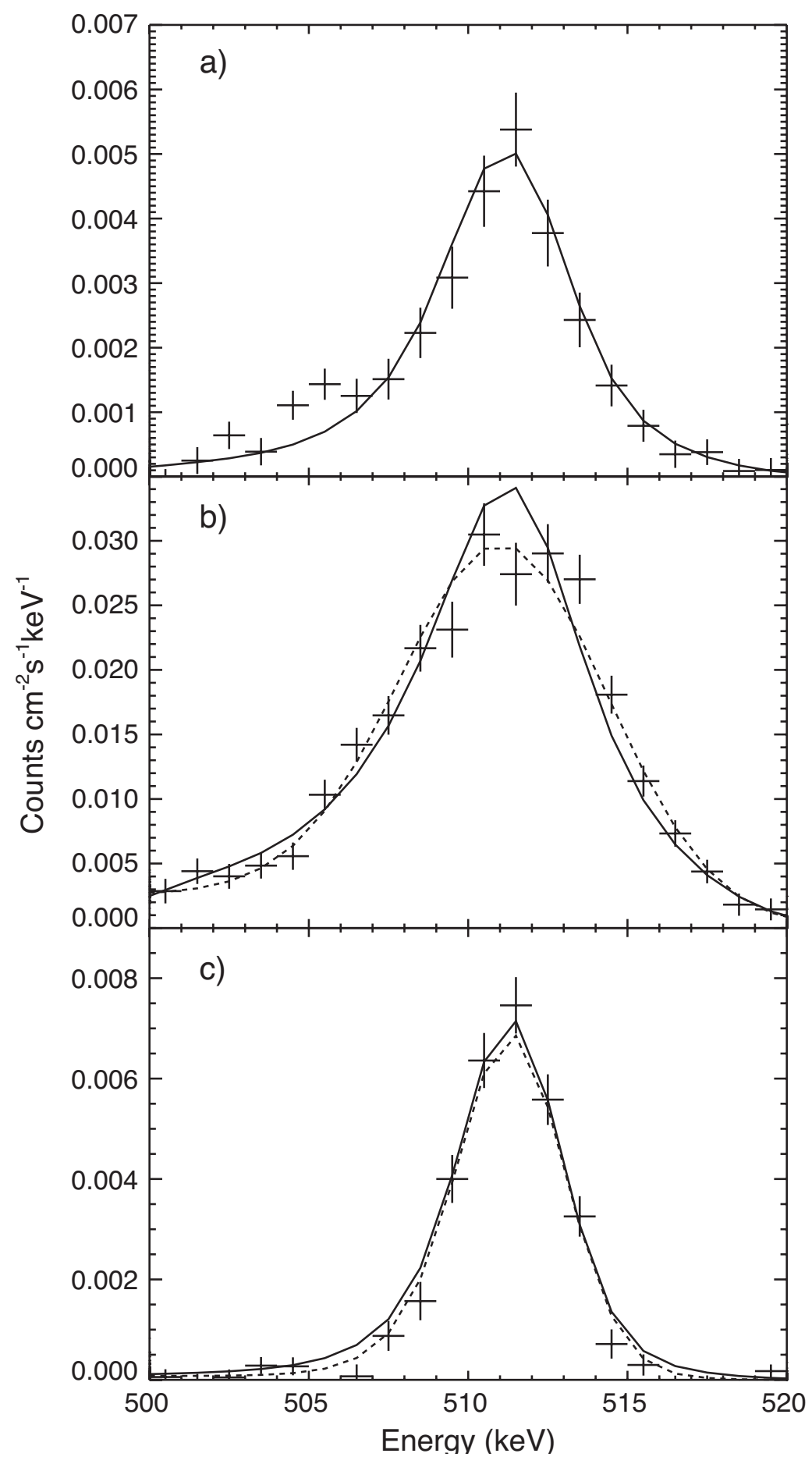

Figure 24 